\title{
The radiative transfer at second order: a full treatment of the Boltzmann equation with polarization
}

\author{
Cyril Pitrou \\ E-mail: cyrilp@astro.uio.no \\ Institute of Theoretical Astrophysics, University of Oslo, P.O. Box 1029 \\ Blindern, 0315 Oslo, Norway. \\ PACS numbers: 98.80.-k, 98.80.Jk, 98.70.Vc, 04.20.Cv
}

\begin{abstract}
This article investigates the full Boltzmann equation up to second order in the cosmological perturbations. Describing the distribution of polarized radiation by a tensor valued distribution function, we study the gauge dependence of the distribution function and summarize the construction of the gauge-invariant distribution function. The Liouville operator which describes the free streaming of electrons, and the collision term which describes the scattering of photons on free electrons are computed up to second order. Finally, the remaining dependence in the direction of the photon momentum is handled by expanding in projected symmetric trace-free multipoles and also in the more commonly used normal modes components. The results obtained remain to be used for computing numerically the contribution in the cosmic microwave background bispectrum which arises from the evolution of second order perturbations, in order to disentangle the primordial non-Gaussianity from the one generated by the subsequent non-linear evolution.
\end{abstract}

\section{Introduction}

The cosmic microwave background (CMB) has become in the past twenty years a central observable of modern cosmology. The properties of the CMB temperature fluctuations depend both on the initial conditions set at the end of the primordial inflationary era, and on their evolution through time in the post-inflationary eras. The theory of cosmological perturbations around a space-time with maximally symmetric spatial sections is a cornerstone of our understanding of the large scale structure of the universe. The relativistic matter (photons, neutrinos) is described with a statistical approach 1, 2, 3, also referred to as kinetic theory, in which we use a distribution function whose evolution is given by a Boltzmann equation. As for non-relativistic matter [baryons, cold dark matter], a fluid approximation is usually sufficient. Given the typical amplitude $\left(10^{-5}\right)$ of the metric fluctuations, the dynamics of the cosmological perturbations was so far mainly studied at linear order around a Friedmann-Lemaitre (FL) space-time, and characterized statistically by the power spectrum. This method allows to relate the CMB angular power spectrum to the initial power spectrum, which opens a window on the early universe. However, the linear order of perturbations fails to capture the intrinsic non-linear features of General Relativity which enter both 
the initial conditions and the evolution. The CMB measurements, though already of high precision 4, 5, are soon going to improve with the new forthcoming missions such as Planck [6], and will be sensitive to these non-linear effects. It becomes thus necessary to go beyond this linear perturbation scheme by studying the second-order perturbations. This is even more crucial if we want to estimate the bispectrum in the cosmic microwave background since this can only arise from non-Gaussian initial conditions set by inflation or from non-linear evolution. In order to improve our theory of the early universe and discriminate between different models of inflation, it is thus necessary to disentangle the primordial non-Gaussianity from the one induced by non-linear evolution. We thus need to extend the program followed at first order in perturbations up to second order.

Since cosmological perturbations are plagued by the gauge freedom, we need to build a full set of gauge invariant perturbation variables and derive the perturbed Boltzmann and Einstein equations in terms of these variables to obtain their dynamical equations. The first-order gauge invariant perturbation variables in the fluid approach were built in [7, and in the kinetic theory in 8, 9. In the inflationary era and for slow-roll one field inflation, the quantization in the linear equations of the canonical degrees of freedom [10] which transfer to Gaussian classical fluctuations enable us to fix the initial conditions for the post-inflationary dynamics on superHubble scales. By using quantities which are conserved for modes larger than the Hubble radius [11, 12, 13, we can ignore the details between the end of inflation and the subsequent eras. In this program, the evolution of radiation 1 requires a special care since the distribution function is shaped by Compton scattering by free electrons and in particular this generates polarization. At first order, the evolution equations describing polarized radiation were studied intensively in 14, 15, 16, and codes have been made available [17, 18, for integrating numerically these equations and thus analyzing the CMB data.

At second-order, the program remains so far incomplete and this paper aims at filling this gap. The quantization of the canonical degrees of freedom in the models of slow-roll one field inflation with non-linear couplings has been investigated in the interaction picture [19, 20] up to the loop corrections [21, 22, 23] aiming at computing the level of primordial non-Gaussianity. This generically predicts negligible amounts of non-Gaussianity [19] whereas multi-field inflation [24, 25, 26, 27, 28, 29, 30, 31, 32 . can generate significant levels of non-Gaussianity. Since the use of adapted estimators 33, 34, 35. tends to show that there might be a detectable amount of nonGaussianity in the CMB [4, 5, 36, the understanding of non-linear evolution is of first importance in order to constrain these models of inflation. In particular all non-linear effects in the foreground [37, 38, 39, 40, (see 41 for a review) have to be understood and estimated. In order to develop the mathematical tools for this general study, the gauge issue in the fluid approach was studied in 42 and gauge invariant variables were built in 12, 43 up to second order in perturbations. This fluid approximation has already been used to understand the general form of the bispectrum generated by evolutionary effects [4]. As for the more general kinetic approach, the gauge issue was studied in our previous paper [45, and the evolution equations through free-streaming

$\ddagger$ Usually, the word radiation is used for relativistic particle. In the context of CMB, it is often used just for photons, as it is the case in this paper. 
and Compton collision on free electrons were derived in 46, 47, 48, 49, but it is so far restricted to unpolarized radiation, which is inconsistent since Compton scattering does generate polarization. In this article, we will extend these works to polarized radiation, leaving open the issue of second-order numerical integration. It should be mentioned that an alternative program consists in working directly with covariantly defined quantities in the so-called $1+3$ covariant formalism. The first order canonical degrees of freedom required to fix the initial conditions in this approach have been identified [50, the conserved quantities, used to ignore the detail between the inflationary era and the subsequent eras, have been built [51, 52] and the dynamical equations up to second order [53, 54, 55, 56, 57, 58, 59] have also been extensively studied, but so far leaving aside for the second order the problem of mode decomposition and the treatment of polarized polarization. The results presented in this paper can be used in a straightforward manner for the description of collisions in this $1+3$ formalism, and we even correct a small mistake for the unpolarized case. The two formalisms should of course lead to the same conclusion and they have been compared in 60, 61, 62.

This paper is organized as follows. We first review the description of polarized radiation with a tensor valued distribution function in section 1 We then recall the gauge dependence and the construction of gauge invariant variables when using a fluid approximation 2. In section 3, we summarize the results of our previous paper [45] which focused on the gauge dependence of the distribution function and the construction of a gauge invariant distribution function in the kinetic theory, and we extend the results to the tensor valued distribution function. This formalism is then used to compute the second order gauge invariant Liouville operator, for radiation in section 4 and for matter in section 5 . The collision term for both cases is computed in section 6. Finally, in section 7 we develop the necessary tools to express these results in terms of the normal modes decomposition.

\section{Kinetic theory}

\subsection{Momentum and tetrad}

In the kinetic description of radiation, the momentum of photons is usually decomposed onto an orthonormal basis, that is using a tetrad field. Though not compulsory, this facilitates the separation between the magnitude of the momentum and its direction represented by a spacelike unit vector. The tetrad vectors $\mathbf{e}_{a}$ and their corresponding tetrad forms $\mathbf{e}^{a}$ satisfy the orthonormality conditions

$$
\mathbf{e}_{a} \cdot \mathbf{e}_{b} \equiv e_{a}^{\mu} e_{b}^{\nu} g_{\mu \nu}=\eta_{a b}, \quad \mathbf{e}^{a} \cdot \mathbf{e}^{b} \equiv e^{a}{ }_{\mu} e^{b} g^{\mu \nu}=\eta^{a b},
$$

where $g_{\mu \nu}$ is the space-time metric, $g^{\mu \nu}$ its inverse and $\eta^{a b}=\eta_{a b}$ the Minkowski metric. In the previous expressions and throughout this paper, we use Greek indices $(\mu, \nu, \rho, \sigma \ldots)$ for abstract indices and the beginning of the Latin alphabet $(a, b, c, d \ldots)$ for tetrad labels. Since the tetrad labels run from 0 to 3, we also use Latin indices starting from the letter $i$ (that is $i, j, k, l \ldots$ ) with values ranging from 1 to 3 to label the spacelike vectors or forms of a tetrad. We then reserve the label $o$ for the timelike vector and form in a tetrad. A momentum can then be decomposed as

$$
\mathbf{p}=p^{a} \mathbf{e}_{a}=p^{o} \mathbf{e}_{o}+p^{i} \mathbf{e}_{i},
$$

where the components can be extracted as

$$
p^{a}=\mathbf{p} \cdot \mathbf{e}^{a} \equiv e_{\mu}^{a} p^{\mu} .
$$


It can be decomposed into a magnitude $p^{o}$ and the a direction vector $\mathbf{n}$ according to

$$
p^{\mu}=p^{o}\left(e_{o}^{\mu}+n^{\mu}\right), \quad \mathbf{n} . \mathbf{e}_{o} \equiv n_{\mu} e_{o}^{\mu}=0, \quad n^{\mu} n_{\mu} \equiv \mathbf{n} . \mathbf{n}=1 .
$$

This decomposition can be used to define a screen projector

$$
S_{\mathbf{e}_{\mathbf{o}} \mu \nu}(\mathbf{p})=g_{\mu \nu}+e_{\mu}^{o} e_{\nu}^{o}-n_{\mu} n_{\nu},
$$

which projects on a space which is both orthogonal to $\mathbf{e}_{o}$ and orthogonal to the direction $\mathbf{n}$, since

$S_{\mathbf{e}_{o} \mu \nu} e_{o}^{\mu}=0, \quad S_{\mathbf{e}_{o} \mu \nu} p^{\mu}=0, \quad S_{\mathbf{e}_{o} \mu \nu} n^{\nu}=0, \quad S_{\mathbf{e}_{o \mu}}{ }^{\nu} S_{\mathbf{e}_{o \nu \sigma}}=S_{\mathbf{e}_{o \mu \sigma}}$.

In this paper, a projected tensorial quantity is orthogonal to $\mathbf{e}_{o}$ only and a screen projected quantity is orthogonal to both $\mathbf{n}$ and $\mathbf{e}_{o}$. If there is no risk of confusion about the choice of the observer in this screen projector, then we will use the notation $S_{\mu \nu}$ instead of $S_{\mathbf{e}_{o} \mu \nu}$. If we also omit the dependence of the screen projector in the photon momentum, then we abbreviate $S_{\mu \nu}(\mathbf{p})$ in $S_{\mu \nu}$, and $S_{\mu \nu}\left(\mathbf{p}^{\prime}\right)$ in $S_{\mu \nu}^{\prime}$. The polarization of a photon is represented by its polarization vector $\boldsymbol{\epsilon}(\mathbf{p})$ which is a complex spacelike unit vector $\left(\epsilon_{\mu}^{\star}(\mathbf{p}) \epsilon^{\mu}(\mathbf{p})=1\right)$ and taken in the Lorentz gauge $\left(\epsilon_{\mu}(\mathbf{p}) p^{\mu}=0\right)$. Since there is a residual gauge freedom (of electromagnetism) in the choice of the polarization vector, we will work with the screen projected polarization vector $S_{\nu}^{\mu} \epsilon^{\nu}(\mathbf{p})$ which is unique.

\subsection{The (screen-projected) polarization tensor}

The radiation is represented by a Hermitian tensor valued distribution function (which is thus complex valued), also called polarization tensor [63, 64, 58, 65] satisfying

$$
F_{\mu \nu}\left(x^{A}, p^{a}\right) \quad p^{\mu} F_{\mu \nu}\left(x^{A}, p^{a}\right)=0,
$$

from which we can form the distribution function of photons in a given state of polarization $\epsilon$ by

$$
F_{\mu \nu}\left(x^{A}, p^{a}\right) \epsilon^{\star \mu}(\mathbf{p}) \epsilon^{\nu}(\mathbf{p}) .
$$

Here the $x^{A}$ are the coordinates used to label points on the space-time manifold. Throughout this paper, indices which refer to these coordinates are $A, B, C$, . if they run from 0 to 3 . Furthermore, indices which are $I, J, K, \ldots$ run from 1 to 3 , and the time component index is $O$.

For a given electromagnetic plane wave with potential vector amplitude $A^{\mu}=A \epsilon^{\mu}$ and wave vector $k^{\mu}$ in the geometric optics limit, this polarization tensor can be defined 66 . by

$$
\delta_{D}^{1}(\mathbf{p} \cdot \mathbf{p}) F_{\mu \nu}\left(p^{a}\right) \equiv \frac{1}{2}(2 \pi)^{3} \delta_{D}^{4}(\mathbf{k}-\mathbf{p}) A^{2} \epsilon_{\mu}(\mathbf{k}) \epsilon_{\nu}^{\star}(\mathbf{k}),
$$

where $\delta_{D}^{n}$ is the Dirac function of dimension $n$. This tensor $F_{\mu \nu}$ should not be confused with the Faraday tensor. Since the remaining gauge freedom of electromagnetism also affects the polarization tensor, we can define the screen-projected distribution function by

$$
f_{\mu \nu}\left(x^{A}, p^{a}\right)=S_{\mu}^{\rho} S_{\nu}^{\sigma} F_{\rho \sigma}\left(x^{A}, p^{a}\right) .
$$

This tensor is no more dependent on the residual gauge freedom and encodes all the polarization properties of radiation as seen by an observer having a velocity $\mathbf{e}_{o}$. Similarly to the definition of the screen projector, if the context requires it, we will use an index notation of the type $f_{\mathbf{e}_{o}}^{\mu \nu}$ to remind with which velocity, and thus with 
which screen projector, it is defined. The screen projected tensor has four degrees of freedom which can be split according to

$$
f_{\mu \nu}\left(x^{A}, p^{a}\right) \equiv \frac{1}{2} I\left(x^{A}, p^{a}\right) S_{\mu \nu}+P_{\mu \nu}\left(x^{A}, p^{a}\right)+\frac{\mathrm{i}}{2} V\left(x^{A}, p^{a}\right) \epsilon_{\mu \nu \sigma} n^{\sigma},(1.1
$$

with $\epsilon_{\mu \nu \sigma} \equiv e_{o}^{\rho} \epsilon_{\rho \mu \nu \sigma}$, and where $\epsilon_{\rho \mu \nu \sigma}$ is the completely antisymmetric tensor. $P_{\mu \nu}$, which encodes the degree of linear polarization, is real symmetric and trace free, as well as orthogonal to $\mathbf{e}_{o}$ and $\mathbf{n}$, and thus encodes two degrees of freedom, which can be described by the Stokes functions $Q$ and $U$ [58. $I\left(x^{A}, p^{a}\right)$ and $V\left(x^{A}, p^{a}\right)$ are respectively the intensity (or distribution function) for both polarizations and the degree of circular polarization. We also define the normalized (screen-projected) polarization tensor by

$$
U_{\mu \nu} \equiv \frac{f_{\mu \nu}}{f_{\alpha}^{\alpha}}=\frac{f_{\mu \nu}}{I} .
$$

\subsection{Stress-energy tensor and energy-integrated functions}

For a distribution of photons, a stress-energy tensor can be defined by

$$
T^{\mu \nu}\left(x^{A}\right) \equiv e_{a}^{\mu} e_{b}^{\nu}\left(\int \delta_{D}^{1}(\mathbf{p} \cdot \mathbf{p}) I\left(x^{A}, p^{c}\right) p^{a} p^{b} \frac{\mathrm{d} p^{o} \mathrm{~d}^{3} p^{i}}{(2 \pi)^{3}}\right) .
$$

Performing the integral over $p^{o}$ (we choose the convention in which we integrate on the two mass hyperboloides), we eliminate the Dirac function and it leads to

$$
T^{\mu \nu}\left(x^{A}\right) \equiv e_{a}^{\mu} e_{b}^{\nu}\left(\int I\left(x^{A}, p^{i}\right) p^{a} p^{b} \frac{\mathrm{d}^{3} p^{i}}{p^{o}(2 \pi)^{3}}\right),
$$

where now the intensity distribution function has to be considered as a function of $p^{i}$, and $p^{o}$ is positive and taken on the mass shell, that is $p^{o}=\sqrt{p_{i} p^{i}}$, and is thus identified with the energy of the photon. Splitting the integral over $\mathrm{d}^{3} p^{i}$ into magnitude and angular direction leads to

$$
T^{\mu \nu}\left(x^{A}\right) \equiv \frac{1}{(2 \pi)^{3}} e_{a}^{\mu} e_{b}^{\nu}\left(\int I\left(x^{A}, p^{i}\right)\left(p^{o}\right)^{3} N^{a} N^{b} \mathrm{~d} p^{o} \mathrm{~d}^{2} \Omega\right),
$$

where $\mathrm{d}^{2} \Omega$ is the differential solid angle associated with the unit vector $n^{i}$, and where $N^{i} \equiv n^{i}$ and $N^{o} \equiv 1$. This motivates the definition of the (energy-) integrated counterparts of $I, V$ and $P_{\mu \nu}$ which are

$$
\begin{aligned}
\mathcal{I}\left(x^{A}, n^{i}\right) & \equiv \frac{4 \pi}{(2 \pi)^{3}} \int I\left(x^{A}, p^{o}, n^{i}\right)\left(p^{o}\right)^{3} \mathrm{~d} p^{o}, \\
\mathcal{V}\left(x^{A}, n^{i}\right) & \equiv \frac{4 \pi}{(2 \pi)^{3}} \int V\left(x^{A}, p^{o}, n^{i}\right)\left(p^{o}\right)^{3} \mathrm{~d} p^{o}, \\
\mathcal{P}_{\mu \nu}\left(x^{A}, n^{i}\right) & \equiv \frac{4 \pi}{(2 \pi)^{3}} \int P_{\mu \nu}\left(x^{A}, p^{o}, n^{i}\right)\left(p^{o}\right)^{3} \mathrm{~d} p^{o} .
\end{aligned}
$$

$\mathcal{I}$ is the brightness, $\mathcal{V}$ is the degree of linear polarization in units of $\mathcal{I}$, and $\mathcal{P}_{\mu \nu}$ is the tensor of linear polarization in units of $\mathcal{I}$. 


\subsection{Description of massive particles}

For massive particles such as electrons (e), protons (p) or cold dark matter (c), we do not need to describe polarization and thus we can rely solely on a the distribution functions $g_{\mathrm{e}}, g_{\mathrm{p}}$ and $g_{\mathrm{c}}$ (chosen to describe the two helicities). Additionally, following common practice in cosmology, we will refer to electrons and protons together as baryons though electrons are leptons. This is motivated by the fact that most of the mass is carried by protons which are baryons, and the Compton interaction between protons and electrons makes these two components highly interdependent. For a particle with impulsion $q^{a}$, we use the following notation

$n^{i} \equiv \frac{q^{i}}{q^{o}}, \quad \beta=\sqrt{n^{i} n_{i}}, \quad \lambda \equiv \beta q^{o}, \quad \hat{n}^{i}=n^{i} / \beta, \quad \gamma=\left(1-\beta^{2}\right)^{-1 / 2}$,

where now we have to distinguish between the unit vector $\hat{n}^{i}$ and the velocity vector $n^{i}$ because of the mass $m$ of the particles. The stress-energy tensor can be defined in a similar manner to equation (1.15) by

$$
T^{\mu \nu}\left(x^{A}\right) \equiv e_{a}^{\mu} e_{b}^{\nu}\left(\int \delta_{D}^{1}\left(\mathbf{q . q}-m^{2}\right) g\left(x^{A}, q^{c}\right) q^{a} q^{b} \frac{\mathrm{d} q^{o} \mathrm{~d}^{3} q^{i}}{(2 \pi)^{3}}\right) .
$$

Integrating over $q^{o}$, this leads to

$$
T^{\mu \nu}\left(x^{A}\right) \equiv \frac{1}{(2 \pi)^{3}} e_{a}^{\mu} e_{b}^{\nu}\left(\int g\left(x^{A}, q^{i}\right) q^{o} N^{a} N^{b} \mathrm{~d}^{3} q^{i}\right),
$$

where we recall that $N^{a} \equiv\left(1, n^{i}\right)$, and $q^{o}$ is taken on the mass shell $\left(q^{o}=\sqrt{q_{i} q^{i}+m^{2}}\right)$.

\subsection{The fluid limit}

The stress-energy tensor of radiation or matter is equivalent to the one of an imperfect fluid with stress-energy tensor

$$
T_{\mu \nu}=\rho u_{\mu} u_{\nu}+P\left(g_{\mu \nu}+u_{\mu} u_{\nu}\right)+\Pi_{\mu \nu} .
$$

In this decomposition $\rho$ is the energy density, $P$ is the pressure, $u^{\mu}$ is the fluid velocity and $\Pi^{\mu \nu}$, which satisfies $\Pi_{\mu}^{\mu}=u^{\mu} \Pi_{\mu \nu}=0$, is the anisotropic stress. For an isotropic distribution of radiation, that is where $\mathcal{I}\left(x^{A}, p^{i}\right)$ depends only on the magnitude of $p^{i}$, it is straightforward to show by comparison of the expressions (1.15) and (1.22) that $P=\rho / 3$. Similarly, for a set of heavy particles (or non-relativistic particles), that is with $\sqrt{q^{i} q_{i}} \ll m$, the pressure satisfies $P \ll \rho$ and the anisotropic stress tensor is also similarly small. For cold dark matter, it is assumed that the mass of particles is large enough so that we can use this approximation.

In the case of electrons and protons, that is baryons, the Coulomb interaction ensures that the distribution of momenta follows a Fermi-Dirac distribution in the reference frame where they have no bulk velocity [2], at least as long as the baryonic matter is ionized. This distribution is isotropic in this adapted frame and depends only on $\lambda$

$$
g_{\mathrm{fd}}(\lambda)=\left(\exp \left[\frac{\sqrt{\left(\lambda^{2}+m^{2}\right)}-\mu}{T}\right]+1\right)^{-1} .
$$


As a consequence, the anisotropic stress vanishes, and in this adapted frame the baryons are then ideally described by the energy density and the pressure

$$
\begin{aligned}
& \rho_{\mathrm{b}} \equiv \frac{4 \pi}{(2 \pi)^{3}} \int g_{\mathrm{fd}}(\lambda) \sqrt{\lambda^{2}+m^{2}} \lambda^{2} \mathrm{~d} \lambda, \\
& P_{\mathrm{b}} \equiv \frac{4 \pi}{3(2 \pi)^{3}} \int g_{\mathrm{fd}}(\lambda) \frac{\lambda^{4}}{\sqrt{\lambda^{2}+m^{2}}} \mathrm{~d} \lambda .
\end{aligned}
$$

If we can neglect the chemical potential $\mu$, which is the case in the cosmological context, then for non-relativistic particles we obtain

$$
\begin{aligned}
& \rho_{\mathrm{b}} \simeq m\left(1+\frac{3 T}{2 m}\right) n_{\mathrm{b}}, \\
& P_{\mathrm{b}} \simeq n_{\mathrm{b}} T .
\end{aligned}
$$

The baryonic matter is ionized roughly until recombination where the temperature of photons is of order $T_{\mathrm{LSS}} \simeq 0.25 \mathrm{eV}$ [5]. For electrons, the thermal correction is of order $T_{\mathrm{LSS}} / m_{\mathrm{e}} \simeq 0.25 / 511000 \simeq 0.510^{-6}$, and this ratio is even $m_{\mathrm{p}} / m_{\mathrm{e}} \simeq 1836$ times smaller for protons. We thus deduce that the baryons either have no anisotropic stress because of Coulomb interaction, or have a very tiny pressure and anisotropic stress after recombination has occurred. However, as discussed in section 6.2 this thermal correction is still of order of the metric perturbations and should not in principle be ignored for second order computations. Nevertheless, the thermal corrections are not relevant for computing the bispectrum and it is for this reason that we will, from now on, drop terms in $T / m$ and describe baryons as cold matter (but not dark since it can interact with radiation). It should be mentionned that for neutrinos these conclusions are not valid anymore since they are very light [67]. We will here assume that they are light enough to be treated as collisionless radiation, and the equations which govern their evolution can be found by setting $\sigma_{\mathrm{T}}=0$ in the equations for photons, where $\sigma_{\mathrm{T}}$ is the Thomson cross section.

\subsection{Multipole expansion for radiation}

Functions of $p^{a}$ can be viewed as functions of $\left(p^{o}, n^{a}\right)$ and we can separate the dependence into the energy and the direction of the momentum. The dependence in the direction can be further expanded in multipoles using projected symmetric trace-free (PSTF) tensors, where projected means that they are orthogonal to $\mathbf{e}_{o}$. For instance, $I$ can be expanded as

$$
I\left(x^{A}, p^{o}, n^{a}\right)=\sum_{\ell=0}^{\infty} \underline{I_{\underline{a_{\ell}}}}\left(x^{A}, p^{o}\right) n \underline{a_{\ell}},
$$

where the $I_{a_{\ell}} \equiv I_{a_{1} \ldots a_{\ell}}$ are PSTF. For the lowest multipole, i.e. the one corresponding to $\ell=0, \overline{\text { we }}$ use the notation $I_{\emptyset}$. Note that we have defined the notation $n \underline{a_{\ell}} \equiv$ $n^{a_{1}} \ldots n^{a_{\ell}}$. We also remind that $n^{a} \equiv \mathbf{n} . \mathbf{e}^{a} \equiv n^{\mu} e_{\mu}^{a}$. Since $\mathbf{n}$ is projected, $n^{o}=0$ and thus if any of the indices in $I_{a_{\ell}}$ is $o$, then the multipoles is chosen to vanish. These multipoles can be obtained by performing the following integrals

$$
I_{\underline{a_{\ell}}}\left(x^{A}, p^{o}\right)=\Delta_{\ell}^{-1} \int I\left(x^{A}, p^{o}, n^{a}\right) n_{\left.\underline{\left\langle a_{\ell}\right.}\right\rangle} \mathrm{d}^{2} \Omega, \quad \Delta_{\ell} \equiv 4 \pi \frac{\ell !}{(2 \ell+1) ! !},
$$

where $\langle\ldots\rangle$ means the symmetric trace-free part. A similar expansion can be performed on $V$, by replacing $I$ by $V$ in the above expressions, as well as for their 
energy integrated counterparts. Note that in particular

$$
\begin{aligned}
& \mathcal{I}^{\emptyset}=T^{o o}, \\
& \mathcal{I}^{i}=4 \pi \Delta_{1}^{-1} T^{o i}, \\
& \mathcal{I}^{i j}=4 \pi \Delta_{2}^{-1} T^{\langle i j\rangle} .
\end{aligned}
$$

As for $P_{a b} \equiv P_{\mu \nu} e_{a}^{\mu} e_{b}^{\nu}$, it can be expanded in electric and magnetic type components according to [54, 58, 68, 69.

$P_{a b}\left(x^{A}, p^{a}\right)=\sum_{\ell=2}^{\infty}\left[E_{a b \underline{c_{\ell-2}}}\left(x^{A}, p^{o}\right) n \underline{c_{\ell-2}}-n_{c} \epsilon_{(a}^{c d} B_{b) \underline{d c_{\ell-2}}}\left(x^{A}, p^{o}\right) n \underline{c_{\ell-2}}\right]^{\mathrm{TT}}$,

where the notation TT denotes the transverse (to $\mathbf{n}$ ) symmetric trace-free part, which for a second rank tensor is

$$
\left[X_{a b}\right]^{\mathrm{TT}} \equiv S_{(a}^{c} S_{b)}^{d} X_{c d}-\frac{1}{2} S_{a b} S^{c d} X_{c d}
$$

The electric and magnetic multipoles can be obtained by performing the integrals

$$
\begin{aligned}
& E_{\underline{a_{\ell}}}\left(x^{A}, p^{o}\right)=M_{\ell}^{2} \Delta_{\ell}^{-1} \int n_{\left\langle\underline{a_{\ell-2}}\right.} P_{\left.a_{\ell-1} a_{\ell}\right\rangle}\left(x^{A}, p^{o}, n^{a}\right) \mathrm{d}^{2} \Omega, \\
& B_{\underline{a_{\ell}}}\left(x^{A}, p^{o}\right)=M_{\ell}^{2} \Delta_{\ell}^{-1} \int n_{b} \epsilon_{\left\langle a_{\ell}\right.}^{b d} n_{a_{\ell-2}} P_{\left.a_{\ell-1}\right\rangle d}\left(x^{A}, p^{o}, n^{a}\right) \mathrm{d}^{2} \Omega,
\end{aligned}
$$

where

$$
M_{\ell}=\sqrt{\frac{2 \ell(\ell-1)}{(\ell+1)(\ell+2)}}
$$

\subsection{Transformation rules under a change of frame}

1.7.1. The photon momentum So far, everything was defined with respect to an observer having a velocity $\mathbf{e}_{o}$. How does all this machinery transform when the radiation is observed by an observer with a different velocity $\tilde{\mathbf{e}}_{o}$ ? Since two velocities can be related by a Lorentz transformation, there exists a vector $\mathbf{v}$ such that

$$
\tilde{\mathbf{e}}_{o}=\gamma\left(\mathbf{e}_{o}+\mathbf{v}\right), \quad \gamma \equiv \frac{1}{\sqrt{1-\mathbf{v} \cdot \mathbf{v}}} \quad v^{o} \equiv \mathbf{v} \cdot \mathbf{e}^{o}=0 .
$$

We deduce immediately that the magnitude and the direction unit vector of the photon momentum transform as

$$
\begin{aligned}
p^{\tilde{o}} & \equiv \mathbf{p} . \tilde{\mathbf{e}}^{o}=\gamma p^{o}(1-\mathbf{n} \cdot \mathbf{v}) \\
\tilde{\mathbf{n}} & =\frac{1}{\gamma(1-\mathbf{v} \cdot \mathbf{n})}\left(\mathbf{e}_{o}+\mathbf{n}\right)-\gamma\left(\mathbf{e}_{o}+\mathbf{v}\right) .
\end{aligned}
$$

We remind that the direction, as observed by the transformed observer, is given by the decomposition $\mathbf{p}=p^{\tilde{o}}\left(\tilde{\mathbf{e}}_{o}+\tilde{\mathbf{n}}\right)$. These rules imply the following transformation rule for the screen projector

$$
\tilde{S}_{\mu \nu}=S_{\mu \nu}+\frac{2 \gamma}{p^{\tilde{o}}} p_{(\mu} S_{\nu) \rho} v^{\rho}+\left(\frac{\gamma}{p^{\tilde{o}}}\right)^{2} p_{\mu} p_{\nu} S_{\alpha \beta} v^{\alpha} v^{\beta}
$$

which implies directly the following useful relations

$$
\tilde{S}_{\mu \nu}=\tilde{S}_{\mu}{ }^{\rho} \tilde{S}_{\nu}{ }^{\sigma} S_{\rho \sigma}, \quad \tilde{n}^{\mu} \tilde{\epsilon}_{\mu \rho \sigma}=n^{\mu} \epsilon_{\mu \alpha \beta} \tilde{S}_{\rho}^{\alpha} \tilde{S}_{\sigma}^{\beta} .
$$


The last relation can also be demonstrated easily by noting that $n^{\mu} \epsilon_{\mu \alpha \beta} \tilde{S}_{\rho}^{\alpha} \tilde{S}_{\sigma}^{\beta}$ is by construction orthogonal to $\tilde{\mathbf{e}}_{o}$ and $\tilde{\mathbf{n}}$, and since it is also obviously antisymmetric in its two free indices, it has to be proportional to $\tilde{n}^{\mu} \tilde{\epsilon}_{\mu \rho \sigma}$. By contracting both expressions with themselves we obtain that they are indeed equal.

The rest of the tetrad can be transformed without rotation, that is with a pure boost, by

$$
\tilde{e}^{a}=\Lambda_{b}^{a} e^{b}, \quad \tilde{e}_{a}=e_{b}\left(\Lambda^{-1}\right)^{b}{ }_{a}=\Lambda_{a}^{b} e_{b},
$$

and the components of this transformation are given by

$$
\Lambda_{o}^{o}=\gamma, \quad \Lambda_{i}^{o}=\Lambda_{o}^{i}=-\gamma v_{i}, \quad \Lambda_{j}^{i}=\delta_{j}^{i}+\frac{\gamma^{2}}{1+\gamma} v^{i} v_{j},
$$

where we remind that $v^{i}$ is the component of $\mathbf{v}$ along the tetrad $\mathbf{e}^{i}$, that is $v^{i}=\mathbf{v} \cdot \mathbf{e}^{i}$. The transformation rule for the photon direction when expressed along tetrads is thus

$$
\tilde{n}^{\tilde{\imath}} \equiv \tilde{\mathbf{n}} \cdot \tilde{\mathbf{e}}^{i}=\frac{1}{\gamma(1-\mathbf{n} \cdot \mathbf{v})}\left[n^{i}+\frac{\gamma^{2}}{(1+\gamma)} \mathbf{n} \cdot \mathbf{v} v^{i}-\gamma v^{i}\right] .
$$

1.7.2. The radiation multipoles It can be easily checked that for a vector orthogonal to $\mathbf{p}$, such as the polarization vector, $\tilde{S}_{\nu}^{\mu}{ }_{\nu}{ }_{\sigma}{ }_{\sigma} \epsilon^{\sigma}=\tilde{S}_{\sigma}^{\mu} \epsilon^{\sigma}$. As an immediate consequence, we deduce from equation (1.9) that the screen-projected polarization tensor transforms according to

$$
\tilde{f}_{\mu \nu}\left(x^{A}, p^{\tilde{o}}, \tilde{n}^{\tilde{a}}\right)=\tilde{S}_{\alpha}^{\mu} \tilde{S}_{\beta}^{\nu} f_{\alpha \beta}\left(x^{A}, p^{o}, n^{a}\right) .
$$

We deduce from equation (1.42) that $P_{\mu \nu}$ transforms following the same rule, whereas $I$ and $V$ transform as scalars

$$
\tilde{I}\left(p^{\tilde{o}}, \tilde{n}^{\tilde{a}}\right)=I\left(p^{o}, n^{a}\right), \quad \tilde{V}\left(p^{\tilde{o}}, \tilde{n}^{\tilde{a}}\right)=V\left(p^{o}, n^{a}\right) .
$$

Here and in the rest of this paper, we omit the dependence in the position $x^{A}$ to simplify the notation. We can deduce from equation (1.39) that the differential solid angle transforms according to (this can also be deduced from using the transformation rule of $p^{o}$ and the fact that $\mathrm{d}^{3} p^{i} / p^{o}=p^{o} \mathrm{~d} p^{o} \mathrm{~d}^{2} \Omega$ is a scalar)

$$
\mathrm{d} \tilde{\Omega}=\left[\frac{1}{\gamma(1-\mathbf{v} \cdot \mathbf{n})}\right]^{2} \mathrm{~d} \Omega
$$

and this can be used to deduce the transformation rules of the multipoles

$\underline{\tilde{\tilde{a}}}_{\underline{\tilde{a}_{\ell}}}\left(p^{\tilde{o}}\right)=\Delta_{\ell}^{-1} \int \mathrm{d} \Omega[\gamma(1-\mathbf{v} \cdot \mathbf{n})]^{-2} \sum_{\ell^{\prime}=0}^{\infty} I_{{\underline{\ell_{\ell}}}^{\prime}}\left[p^{\tilde{o}} \gamma^{-1}\left(1-n^{c} v_{c}\right)^{-1}\right] n \underline{b_{\ell^{\prime}}} \tilde{n}_{\left\langle\underline{\left.\tilde{a}_{\ell}\right\rangle}\right.}$.

In the previous integral, $I_{b_{\ell^{\prime}}}\left[p^{\tilde{o}} \gamma^{-1}\left(1-n^{c} v_{c}\right)^{-1}\right]$ has to be considered as a function of the direction $n^{a}$. It is thus necessary to Taylor expand it as

$$
I_{\underline{b_{\ell}}}\left[p^{\tilde{o}} \gamma^{-1}\left(1-n^{c} v_{c}\right)^{-1}\right]=\sum_{n=0}^{\infty} \frac{1}{n !}\left(\frac{\gamma^{-1} n^{c} v_{c}}{1-\gamma^{-1} n^{c} v_{c}}\right)^{n} I_{\underline{b_{\ell}}}^{\{n\}}\left[p^{\tilde{o}} \gamma^{-1}\right],
$$

where we define

$$
I_{\underline{b_{\ell}}}^{\{n\}}\left(p^{o}\right) \equiv\left(p^{o}\right)^{n} \frac{\partial^{n} I_{b_{\ell}}\left(p^{o}\right)}{\partial\left(p^{o}\right)^{n}},
$$


with the conventions $I_{\underline{b_{\ell}}}^{\prime} \equiv I_{\underline{b_{\ell}}}^{\{1\}}$ and $I_{\underline{b_{\ell}}}^{\prime \prime} \equiv I_{\underline{b_{\ell}}}^{\{2\}}$. Under this form, it is then possible to perform the integration using the following well known integrals [70]

$$
\int n^{i_{1}} \ldots n^{i_{k}} \frac{\mathrm{d}^{2} \Omega}{4 \pi}= \begin{cases}0, & \text { if } k=2 p+1, \\ \frac{1}{k+1}\left[\delta^{\left(i_{1} i_{2}\right.} \ldots \delta^{\left.i_{(k-1)} i_{k}\right)}\right], & \text { if } k=2 p .\end{cases}
$$

Note that we have used the standard notation (...) for the symmetrization of indices which leaves unchanged an symmetric tensor and we will also use the notation [...] for the antisymmetrization of indices which leaves unchanged an antisymmetric tensor. The integrals (1.52) used in the transformation rule (1.49) are ideally suited for a tensor calculus package, and we used xAct [71] to compute them.

Since the result of equation (1.49) will involve terms like $I_{\underline{b_{\ell}}}^{\{n\}}\left[p^{\tilde{o}} \gamma^{-1}\right]=I_{\underline{b_{\ell}}}^{\{n\}}\left[p^{o}(1-\right.$ $\left.n^{c} v_{c}\right)$ ], it will require an additional Taylor expansion in order to have the result expressed only in function of the $I_{\underline{b_{\ell}}}^{\{n\}}\left[p^{\tilde{o}}\right]$ or $I_{\underline{b_{\ell}}}^{\{n\}}\left[p^{o}\right]$. Note that the expression of $\tilde{I}_{\tilde{a}_{\ell}}\left(p^{\tilde{o}}\right)$ in function of the $I_{b_{\ell}}^{\{n\}}\left[p^{\tilde{o}}\right]$, which is the choice that we make in the expressions that we report below, does not depend on the direction $n^{i}$, whereas it does when expressed in function of the $I_{\underline{b_{\underline{e}}}}^{\{n\}}\left[p^{o}\right]$ since $p^{o}$ is unambiguously defined from $p^{\tilde{o}}$ only once a direction $n^{i}$ is specified. A similar method, with similar definitions can be used to determine the transformation rules of the electric and magnetic multipoles [58. At first order in the velocity $\mathbf{v}$, we obtain the following transformation rules

$$
\begin{aligned}
& \tilde{I}_{\underline{\tilde{a}_{\ell}}}\left(p^{\tilde{o}}\right)=I_{\underline{a_{\ell}}}\left(p^{\tilde{o}}\right)+\frac{(\ell+2)(\ell+1)}{(2 \ell+3)} v^{b} I_{b \underline{a_{\ell}}}\left(p^{\tilde{o}}\right)+\frac{(\ell+1)}{(2 \ell+3)} v^{b} I_{b \underline{a_{\ell}}}^{\prime}\left(p^{\tilde{o}}\right) \\
& -(\ell-1) v_{\left\langle a_{\ell}\right.} \underline{I_{\left.a_{\ell-1}\right\rangle}}\left(p^{\tilde{o}}\right)+v_{\left\langle a_{\ell}\right.} I_{\left.a_{\ell-1}\right\rangle}^{\prime}\left(p^{\tilde{o}}\right), \\
& \tilde{E}_{\underline{\tilde{a}_{\ell}}}\left(p^{\tilde{o}}\right)=E_{\underline{a_{\ell}}}\left(p^{\tilde{o}}\right)+\frac{(\ell-1)(\ell+2)(\ell+3)}{(\ell+1)(2 \ell+3)} v^{b} E_{b \underline{a_{\ell}}}\left(p^{\tilde{o}}\right)+\frac{(\ell-1)(\ell+3)}{(\ell+1)(2 \ell+3)} v^{b} E_{b \underline{a_{\ell}}}^{\prime}\left(p^{\tilde{o}}\right) \\
& -(\ell-1) v_{\left\langle a_{\ell}\right.} E_{\left.a_{\ell-1}\right\rangle}\left(p^{\tilde{o}}\right)+v_{\left\langle a_{\ell}\right.} E_{\underline{a_{\ell-1}}}^{\prime}\left(p^{\tilde{o}}\right) \\
& -\frac{2}{(\ell+1)} v_{b} \epsilon_{\left\langle a_{\ell}\right.}^{b c} B_{\left.\underline{a_{\ell-1}}\right\rangle c}\left(p^{\tilde{o}}\right)-\frac{2}{(\ell+1)} v_{b} \epsilon_{\left\langle a_{\ell}\right.}^{b c} B_{\left.\underline{a_{\ell-1}}\right\rangle c}^{\prime}\left(p^{\tilde{o}}\right), \\
& \tilde{B}_{\underline{\tilde{a}_{\ell}}}\left(p^{\tilde{o}}\right)=B_{\underline{a_{\ell}}}\left(p^{\tilde{o}}\right)+\frac{(\ell-1)(\ell+2)(\ell+3)}{(\ell+1)(2 \ell+3)} v^{b} B_{b \underline{a_{\ell}}}\left(p^{\tilde{o}}\right)+\frac{(\ell-1)(\ell+3)}{(\ell+1)(2 \ell+3)} v^{b} B_{b \underline{a_{\ell}}}^{\prime}\left(p^{\tilde{o}}\right) \\
& -(\ell-1) v_{\left\langle a_{\ell}\right.} B_{\left.a_{\ell-1}\right\rangle}\left(p^{\tilde{o}}\right)+v_{\left\langle a_{\ell}\right.} B_{\underline{\left.a_{\ell-1}\right\rangle}}^{\prime}\left(p^{\tilde{o}}\right) \\
& +\frac{2}{(\ell+1)} v_{b} \epsilon_{\left\langle a_{\ell}\right.}^{b c} E_{\left.\underline{a_{\ell-1}}\right\rangle c}\left(p^{\tilde{o}}\right)+\frac{2}{(\ell+1)} v_{b} \epsilon_{\left\langle a_{\ell}\right.}^{b c} E_{\left.\underline{a_{\ell-1}}\right\rangle c}^{\prime}\left(p^{\tilde{o}}\right) .
\end{aligned}
$$

As for the energy-integrated counterparts, they transform at first order in $\mathbf{v}$ as

$$
\begin{aligned}
& \tilde{\mathcal{I}}_{\underline{\tilde{a}_{\ell}}}=\mathcal{I}_{\underline{a_{\ell}}}+\frac{(\ell-2)(\ell+1)}{(2 \ell+3)} v^{b} \mathcal{I}_{\underline{\underline{a_{\ell}}}}-(\ell+3) v_{\left\langle a_{\ell}\right.} \underline{\mathcal{I}}_{\underline{\left.a_{\ell-1}\right\rangle}}, \\
& \tilde{\mathcal{E}}_{\underline{\tilde{a}_{\ell}}}=\mathcal{E}_{\underline{a_{\ell}}}-\frac{(\ell-1)(\ell-2)(\ell+3)}{(\ell+1)(2 \ell+3)} v^{b} \mathcal{E}_{b \underline{a_{\ell}}}-(\ell+3) v_{\left\langle a_{\ell}\right.} \mathcal{E}_{\underline{\left.a_{\ell-1}\right\rangle}}-\frac{6}{(\ell+1)} v_{b} \epsilon^{b c}{ }_{\left\langle a_{\ell}\right.} \mathcal{B}_{\left.\underline{a_{\ell-1}}\right\rangle},
\end{aligned}
$$

and

$\tilde{\mathcal{B}}_{\underline{\tilde{a}_{\ell}}}=\mathcal{B}_{\underline{a_{\ell}}}-\frac{(\ell-1)(\ell-2)(\ell+3)}{(\ell+1)(2 \ell+3)} v^{b} \mathcal{B}_{\underline{b a_{\ell}}}-(\ell+3) v_{\left\langle a_{\ell}\right.} \mathcal{B}_{\underline{\left.a_{\ell-1}\right\rangle}}+\frac{6}{(\ell+1)} v_{b} \epsilon^{b c}{ }_{\left\langle a_{\ell}\right.} \mathcal{E}_{\left.a_{\ell-1}\right\rangle} c$.

We report in Appendix A the transformation rules up to second order in $\mathbf{v}$ for multipoles of further interest. 


\subsection{The Liouville equation}

In this section, we present the equation which governs the free-streaming of photons directly in the tetrad basis though nothing prevents it from being expressed with formal indices. The evolution of the polarization tensor is dictated by the Boltzmann equation

$$
L\left[f_{a b}\left(x^{A}, p^{o}, n^{i}\right)\right]=C_{a b}\left(x^{A}, p^{o}, n^{i}\right) .
$$

$L$ is the Liouville operator whose action on TT tensors like the screen-projected polarization tensor $f_{a b}$ is given by [58]

$L\left[f_{a b}\left(x^{A}, p^{a}\right)\right] \equiv S_{a}^{c} S_{b}^{d}\left[p^{h} \nabla_{h} f_{c d}\left(x^{A}, p^{a}\right)+\frac{\partial f_{c d}\left(x^{A}, p^{a}\right)}{\partial p^{h}} \frac{\mathrm{d} p^{h}}{\mathrm{~d} s}\right]$,

where $s$ is the affine parameter along the particle geodesic. $\nabla$ is the covariant derivative which in the tetrad basis is related to the partial derivative by

$p^{h} \nabla_{h} f_{c d}\left(x^{A}, p^{a}\right) \equiv p^{h} \partial_{h} f_{c d}\left(x^{A}, p^{a}\right)+p^{h} \omega_{h c}^{b} f_{b d}\left(x^{A}, p^{a}\right)+p^{h} \omega_{h d}^{b} f_{c b}\left(x^{A}, p^{a}\right)$,

where the $\omega_{a b c}$ are the Ricci rotation coefficients (see Appendix Cland 72 for details). $C_{a b}$ is the collision tensor whose expression will be detailed in section 6 .

In the case where the collision tensor can be ignored, that is when the collision of photons with electrons or protons can be neglected [the latter type of collision can be always ignored compared to the former since its cross section is reduced by a factor $\left.\left(m_{\mathrm{e}} / m_{\mathrm{p}}\right)^{2}\right]$, this reduces to the Liouville equation. The Liouville equation arises from the fact that the polarization vector $\epsilon$ of a photon is parallel transported and thus satisfies $p^{\mu} \nabla_{\mu} \epsilon^{\nu}=0$, and then it follows from the construction (1.9) of the (not screen projected) polarization tensor $F_{\mu \nu}$ that

$$
p^{h} \nabla_{h} F_{c d}\left(x^{A}, p^{a}\right)+\frac{\partial F_{c d}\left(x^{A}, p^{a}\right)}{\partial p^{h}} \frac{\mathrm{d} p^{h}}{\mathrm{~d} s}=0 .
$$

By using the expression (1.5) for $S^{\mu \nu}$ and the property $F_{\mu \nu}\left(x^{A}, p^{a}\right) p^{\mu}=0$, we obtain directly from the previous equation that the screen-projected tensor satisfies $L\left[f_{a b}\left(x^{A}, p^{a}\right)\right]=0$. It can also be shown that the Liouville operator preserves the decomposition of $f_{\mu \nu}$ in an antisymmetric part $(V)$, a trace $(I)$ and a symmetric traceless part $\left(P_{\mu \nu}\right)$, that is

$L\left[f_{a b}\left(x^{A}, p^{a}\right)\right]=\frac{1}{2} L\left[I\left(x^{A}, p^{d}\right)\right] S_{a b}+L\left[P_{a b}\left(x^{A}, p^{a}\right)\right]+\frac{\mathrm{i}}{2} L\left[V\left(x^{A}, p^{d}\right)\right] n^{c} \epsilon_{c a b}$,

with the Liouville operator acting on a scalar valued function like $I$ or $V$ according to

$$
L\left[I\left(x^{A}, p^{a}\right)\right] \equiv p^{h} \partial_{h} I\left(x^{A}, p^{a}\right)+\frac{\partial I\left(x^{A}, p^{a}\right)}{\partial p^{h}} \frac{\mathrm{d} p^{h}}{\mathrm{~d} s} .
$$

To see this, we need only to use the property $\omega_{a[b c]}=\omega_{a b c}$ and remark that

$$
\begin{aligned}
& S_{(a}^{c} S_{b)}^{d} p^{h} \omega_{h c f} S_{d}^{f}=p^{h} \omega_{h c f} S_{(a}^{c} S_{b)}^{f}=0, \\
& S_{[a}^{c} S_{b]}^{d} p^{h} \omega_{h c f} \epsilon_{d}^{f} \propto S_{[a}^{c} S_{b]}^{d} \epsilon_{c f} \epsilon_{d}^{f}=\epsilon_{f[a} \epsilon_{b]}^{f}=0,
\end{aligned}
$$

where we have used the definition $\epsilon_{\mu \nu} \equiv n^{\alpha} \epsilon_{\alpha \mu \nu}$.

Additionally, since the affine parameter $s$ is a scalar, the transformation properties of the Liouville operator and the collision tensor under a local change of frame is the same as the transformation property of $f_{\mu \nu}$ given in equation (1.46) [58]. 


\section{Gauge transformations and gauge invariance for tensors}

The formalism presented in the previous section is very general and can be applied to the description of matter and radiation in any type of space-time. In the cosmological context it proves useful to use the high symmetries of the large scale space-time to find the solutions of the Einstein and Boltzmann equations using a perturbation scheme. We will review the standard perturbation theory for tensors in this section as well as for a scalar valued distribution function in section 3 and extend it to the TV distribution function.

\subsection{First- and second-order perturbations}

We assume that, at lowest order, the universe is well described by a FriedmannLemaître space-time (FL) with Euclidian spatial sections. The most general form of the metric for an almost FL universe is

$$
\begin{aligned}
\mathrm{d} s^{2} & =g_{\mu \nu} \mathrm{d} x^{\mu} \mathrm{d} x^{\nu} \\
& =a(\eta)^{2}\left\{-(1+2 \Phi) \mathrm{d} \eta^{2}+2 \omega_{I} \mathrm{~d} x^{I} \mathrm{~d} \eta+\left[(1-2 \Psi) \delta_{I J}+h_{I J}\right] \mathrm{d} x^{I} \mathrm{~d} x^{J}\right\},
\end{aligned}
$$

where $\eta$ is the conformal time for which the corresponding index is $O$, and $a(\eta)$ is the scale factor. We perform a scalar-vector-tensor (SVT) decomposition as

$$
\begin{aligned}
& \omega_{I}=\partial_{I} B+B_{I}, \\
& h_{I J}=2 H_{I J}+\partial_{I} E_{J}+\partial_{J} E_{I}+2 \partial_{I} \partial_{J} E,
\end{aligned}
$$

where $B_{I}, E_{I}$ and $H_{I J}$ are transverse $\left(\partial^{I} E_{I}=\partial^{I} B_{I}=\partial^{I} H_{I J}=0\right)$, and $H_{I J}$ is traceless $\left(H_{I}^{I}=0\right)$. There are four scalar degrees of freedom $(\Phi, \Psi, B, E)$, four vector degrees of freedom $\left(B_{I}, E_{I}\right)$ and two tensor degrees of freedom $\left(H_{I J}\right)$. As we shall see in section 2.3 , the perturbation variables live on the background spacetime and thus their indices are lowered and raised by the background (conformally transformed) spatial metric and its inverse, that is with $\delta^{I J}$ and $\delta_{I J}$. Each of these perturbation variables can be split in first and second-order parts as

$$
W=W^{(1)}+\frac{1}{2} W^{(2)}
$$

This expansion scheme will refer, as we shall see, to the way gauge transformations and gauge-invariant (GI) variables are defined. First-order variables are solutions of first-order equations which have been extensively studied (see [73] for a review). Second-order equations will involve purely second-order terms, e.g. $W^{(2)}$ and terms quadratic in the first-order variables, e.g. $\left[W^{(1)}\right]^{2}$. There will thus never be any ambiguity about the order of perturbation variables involved as long as the order of the equation considered is known. Consequently, we will often omit to specify the order superscript when there is no risk of confusion.

As we shall see in section 2.3. 4 of the 10 metric perturbations are gauge degrees of freedom and the 6 remaining degrees of freedom reduce to 2 scalars, 2 vectors and 2 tensors. The three types of perturbations decouple at first order and can thus be treated separately. As long as no vector source terms are present, which is generally the case when no magnetic field or topological defect is taken into account, the first order vector modes decay as $a^{-2}$. Thus, we can safely discard them and set $E_{I}^{(1)}=B_{I}^{(1)}=0$. In the following of this work, we shall not include first-order vector modes for the sake of clarity. We checked that our arguments and derivation can trivially (but at the expense of much lengthy expressions) take them into account. 
In the fluid description, the four-velocity of each fluid is decomposed as

$$
u^{A}=\frac{1}{a}\left(\delta_{O}^{A}+V^{A}\right) .
$$

The indices of $V^{A}$ are raised and lowered with the (conformally transformed) background metric, that is with $\eta_{A B}$ and $\eta^{A B}$. The perturbation $V^{A}$ has only three independent degrees of freedom since $\mathbf{u}$ must satisfy $u_{\mu} u^{\mu}=-1$. The spatial components can be decomposed as

$$
V^{I}=\partial^{I} V+\tilde{V}^{I}
$$

$\tilde{V}^{I}$ being the vector degree of freedom $\left(\partial_{I} \tilde{V}^{I}=0\right)$, and $V$ the scalar degree of freedom. As for the energy density and pressure, similarly to any quantity which does not vanish in the background space-time, they are decomposed according to

$$
\rho=\bar{\rho}+\rho^{(1)}+\frac{1}{2} \rho^{(2)}+\ldots, \quad P=\bar{P}+P^{(1)}+\frac{1}{2} P^{(2)}+\ldots .
$$

It is also common practice to define a conformally transformed anisotropic stress by

$$
\pi^{C D}=\frac{1}{a^{2}} \Pi^{C D}, \quad \pi_{D}^{C}=\Pi_{D}^{C}, \quad \pi_{C D}=a^{2} \Pi_{C D} .
$$

It should be mentionned that due to the symmetries of the background space-time, the anisotropic stress is already a perturbed quantity. At first order, the formalism developed by the seminal work of [7] provides a full set of gauge-invariant variables. Thanks to the general covariance of the equations at hand (Einstein equations, conservation equations, Boltzmann equation), it was shown that it was possible to get first-order equations involving only these gauge-invariant variables. In addition, if these gauge invariant variables reduce, in a particular gauge, to the perturbation variables that we use in this particular gauge, then the computation of the equation can be simplified. Actually, we only need to derive the equations in this particular gauge, as long as it is completely fixed, and then to promote by identification our perturbation variables to the gauge-invariant variables. Thus, provided we know this full set of gauge invariant variables, the apparent loss of generality by fixing the gauge in a calculation, is in fact just a way to simplify computations. Eventually we will reinterpret the equations as being satisfied by gauge invariant variables. The full set of first-order gauge-invariant variables is well known and is reviewed in 73 and 74 . As gauge transformations up to any order were developed, it remained uncertain [42, whether or not a full set of gauge-invariant variables could be built for second and higher orders. This has been recently clarified [43, and the autosimilarity of the transformation rules for different orders can be used as a guide to build the gaugeinvariant variables at any order. We present a summary of the ideas presented in 42 . about gauge transformations and the construction of gauge-invariant variables [43] in a shorter version than in our previous paper [45. A summary emphasizing the differences between the active and passive point of views can also be found in 75 .

\subsection{Points identification on manifolds}

When working with perturbations, we consider two manifolds: a background manifold, $\mathcal{M}_{0}$, with associated metric $\bar{g}$, which in our case is the FL space-time, and the physical space-time $\mathcal{M}_{1}$ with the metric $g$. Considering the variation of metric boils down to a comparison between tensor fields on distinct manifolds. Thus, in order to give a sense to " ${ }^{(1)}(P)=g(P)-\bar{g}(\bar{P})$ ", we need to identify the points $P$ and $\bar{P}$ between these 
two manifolds and also to set up a procedure for comparing tensors. This will also be necessary for the comparison of any tensor field.

One solution to this problem [42] is to consider an embedding $4+1$ dimensional manifold $\mathcal{N}=\mathcal{M} \times[0,1]$, endowed with the trivial differential structure induced, and the projections $\mathcal{P}_{\lambda}$ on submanifolds with $\mathcal{P}_{0}(\mathcal{N})=\mathcal{M} \times\{0\}=\mathcal{M}_{0}$ and $\mathcal{P}_{1}(\mathcal{N})=\mathcal{M} \times\{1\}=\mathcal{M}_{1}$. The collection of $\mathcal{M}_{\lambda} \equiv \mathcal{P}_{\lambda}(\mathcal{N})$ is a foliation of $\mathcal{N}$, and each element is diffeomorphic to the physical space-time $\mathcal{M}_{1}$ and the background space-time $\mathcal{M}_{0}$. The gauge choice on this stack of space-times is defined as a vector field $X$ on $\mathcal{N}$ which satisfies $X^{4}=1$ (the component along the space-time slicing $\mathbb{R}$ ). A vector field defines integral curves that are always tangent to the vector field itself, hence inducing a one parameter group of diffeomorphisms $\phi(\lambda,$.$) , also noted \phi_{\lambda}($.$) ,$ a flow, leading in our case from $\phi\left(0, p \in \mathcal{P}_{0}(\mathcal{N})\right)=p \in \mathcal{P}_{0}(\mathcal{N})$ along the integral curves to $\phi\left(1, p \in \mathcal{P}_{0}(\mathcal{N})\right)=q \in \mathcal{P}_{1}(\mathcal{N})$. Due to the never vanishing last component of $X$, the integral curves will always be transverse to the stack of space-times and the points lying on the same integral curve, belonging to distinct space-times, will be identified. Additionally the property $X^{4}=1$ ensures that $\phi_{\lambda, X}\left(\mathcal{P}_{0}(\mathcal{N})\right)=\mathcal{P}_{\lambda}(\mathcal{N})$, i.e. the flow carries a space-time slice to another. This points identification is necessary when comparing tensors, but we already see that the arbitrariness in the choice of a gauge vector field $X$ should not have physical meaning, and this is the well known gauge freedom.

\subsection{Gauge transformations and gauge invariance}

The induced transport, along the flow, of tensors living on the tangent bundle, is determined by the push-forward $\phi_{\star \lambda}$ and the pull-back $\phi_{\lambda}^{\star}$ [72, 75] associated with an element $\phi_{\lambda}$ of the group of diffeomorphisms. These two functions encapsulate the transformation properties of the tangent and co-tangent spaces at each point and its image. Indeed, the pull-back can be linked to the local differential properties of the vector field embedded by the Lie derivatives along the vector field in a Taylor-like fashion (see [72] or 42])

$$
\phi_{X, \lambda}^{\star}(T)=\sum_{k=0}^{k=\infty} \frac{\lambda^{k}}{k !} \mathcal{L}_{X}^{k} T,
$$

for any tensor $T$. The expansion of equation (2.9) on $\mathcal{P}_{0}(\mathcal{N})$ provides a way to compare a tensor field on $\mathcal{P}_{\lambda}(\mathcal{N})$ to the corresponding one on the background space-time $\mathcal{P}_{0}(\mathcal{N})$. The background value being $\left.T_{0} \equiv \mathcal{L}_{X}^{0} T\right|_{\mathcal{P}_{0}(\mathcal{N})}$, we obtain a natural definition for the tensor perturbation

$$
\left.\Delta_{X} T_{\lambda} \equiv \sum_{k=1}^{k=\infty} \frac{\lambda^{k}}{k !} \mathcal{L}_{X}^{k} T\right|_{\mathcal{P}_{0}(\mathcal{N})}=\phi_{X, \lambda}^{\star}(T)-T_{0} .
$$

The subscript $X$ reminds the gauge dependence. We can read the $n$-th order perturbation as

$$
\left.{ }_{X} T^{(n)} \equiv \mathcal{L}_{X}^{n} T\right|_{\mathcal{P}_{0}(\mathcal{N})},
$$

which is consistent with the expansion of perturbation variables of the physical metric in equation (2.4), since the physical space-time is labeled by $\lambda=1$. However, the fact that the intermediate space-time slices $\mathcal{P}_{\lambda}(\mathcal{N})$ are labeled by $\lambda$ removes the absolute meaning of order by order perturbations, as it can be seen from equation (2.10). The 
entire structure embedded by $\mathcal{N}$ is more than just a convenient construction and this will have important consequences in gauge changes as we will now detail.

If we consider two gauge choices $X$ and $Y$, a gauge transformation from $X$ to $Y$ is defined as the diffeomorphism

$$
\phi_{X \rightarrow Y, \lambda}=\left(\phi_{X, \lambda}\right)^{-1}\left(\phi_{Y, \lambda}\right),
$$

and it induces a pull-back which carries the tensor $\Delta_{X} T_{\lambda}$, which is the perturbation in the gauge $X$, to $\Delta_{Y} T_{\lambda}$, which is the perturbation in gauge $Y$. As demonstrated in [42, 62 this family (indexed by $\lambda$ ) of gauge transformations fails to be a one parameter group due to the lack of the composition rule. It should be Taylor expanded using the so called knight-diffeormorphism along a sequence of vector fields $\xi_{n}$. For the two first orders, the expression of this knight-diffeomorphism is

$$
\begin{aligned}
\phi_{Y, \lambda}^{\star}(T) & =\phi_{X \rightarrow Y, \lambda}^{\star} \phi_{X, \lambda}^{\star}(T) \\
& =\phi_{X, \lambda}^{\star}(T)+\lambda \mathcal{L}_{\xi_{1}} \phi_{X, \lambda}^{\star}(T)+\frac{\lambda^{2}}{2 !}\left(\mathcal{L}_{\xi_{2}}+\mathcal{L}_{\xi_{1}}^{2}\right) \phi_{X, \lambda}^{\star}(T)+\ldots
\end{aligned}
$$

The vector fields $\xi_{1}, \xi_{2}$ are related to the gauge vector fields $X$ and $Y$ by $\xi_{1}=Y-X$ and $\xi_{2}=[X, Y]$. By substitution of the perturbation by its expression in equation (2.10), we identify order by order in $\lambda$, and obtain the transformation rules for perturbations order by order. The first and second order transformation rules, on which we will focus our attention, are

$$
\begin{aligned}
{ }_{Y} T^{(1)}-{ }_{X} T^{(1)} & =\mathcal{L}_{\xi_{1}} T_{0}, \\
{ }_{Y} T^{(2)}-{ }_{X} T^{(2)} & =2 \mathcal{L}_{\xi_{1}} T^{(1)}+\left(\mathcal{L}_{\xi_{2}}+\mathcal{L}_{\xi_{1}}^{2}\right) T_{0} .
\end{aligned}
$$

General covariance, i.e. the fact that physics should not depend on a particular choice of coordinates is an incentive to work with gauge-invariant quantities. As we notice from equation (2.14), a tensor $T$ is gauge-invariant up to $n$-th order if it satisfies $\mathcal{L}_{\xi X} T^{(r)}=0$ for any vector field $\xi$ and any $r \leq n$, as can be deduced by recursion. A consequence of this strong condition is that a tensor is gauge-invariant up to order $n$ if and only if $T_{0}$ and all its perturbations of order lower than $n$ either vanish, or are constant scalars, or are combinations of Kronecker deltas with constant coefficients. Einstein equation is of the form $G-T=0$, and for this reason is totally gauge invariant. However, we cannot find non-trivial tensorial quantities (that is, different from $G-T$ ) gauge-invariant up to the order we intend to study perturbations, with which we could express the perturbed set of Einstein equations.

Consequently, we will build, by combinations of perturbed tensorial quantities, gauge-invariant variables. These combinations will not be the perturbation of an underlying tensor. This method will prove to be very conclusive since a general procedure exists for perturbations around FL. Eventually we shall identify observables among these gauge-invariant variables and the fact that they are not the perturbation of a tensor will not matter. It has to be emphasized that the transformation rules of these combinations are not intrinsic and cannot be deduced directly from the knightdiffeomorphism since they are not tensorial quantities. Instead, we have to form the combination before and after the gauge change in order to deduce their transformation rules.

We now summarize the standard way to build gauge-invariant variables up to second order. For simplicity we will not consider the vector part of the gauge transformations at first order, since we will not consider first order vector modes 
in the metric and fluid perturbation variables (again, this could be done, but would just obfuscate the explanations). In the following, we split $\xi_{1}^{\mu}$ and $\xi_{2}^{\mu}$ as

$$
\xi_{1}{ }^{O}=T^{(1)}, \quad \xi_{1}^{I}=\partial^{I} L^{(1)}, \quad \xi_{2}{ }^{O}=T^{(2)}, \quad \xi_{2}^{I}=\partial^{I} L^{(2)}+L^{I(2)}
$$

with $\partial_{I} L^{I(2)}=0$. We conventionally choose to lower and raise the indices of $\partial^{I} L+L^{I}$ with the (conformally transformed) background metric, which implies that

$$
\xi_{1 I}=a^{2} \partial_{I} L^{(1)}, \quad \xi_{2 I}=a^{2} \partial_{I} L^{(2)}+a^{2} L_{I}^{(2)} .
$$

\subsection{First-order gauge-invariant variables}

In the subsequent work we present the transformation rules of perturbed quantities in a simplified notation. Instead of writing ${ }_{Y} W^{(r)}={ }_{X} W^{(r)}+f\left(\xi_{1}, . ., \xi_{r}\right)$, in order to state that the difference between the expression of the $r$-th order pertubed variable $W$ in gauge $Y$ and in gauge $X$ is a function $f$ of the knight-diffeomorphism fields $\xi_{1}, \ldots, \xi_{r}$, we prefer to write $W^{(r)} \rightarrow W^{(r)}+f\left(\xi_{1}, . ., \xi_{r}\right)$. We remind that the expressions of the fields $\left(\xi_{n}\right)_{1 \leq n \leq r}$ necessary for the knight-diffeomorphism are expressed in function of the gauge fields $X$ and $Y$ [see below equation (2.14)]. From the transformation rules (2.14) we deduce that the first-order perturbations of the metric tensor (2.1) transform as

$$
\begin{aligned}
& \Phi^{(1)} \rightarrow \Phi^{(1)}+T^{(1)}+\mathcal{H} T^{(1)} \\
& B^{(1)} \rightarrow B^{(1)}-T^{(1)}+L^{\prime(1)} \\
& \Psi^{(1)} \rightarrow \Psi^{(1)}-\mathcal{H} T^{(1)} \\
& E^{(1)} \rightarrow E^{(1)}+L^{(1)} \\
& H_{I J}^{(1)} \rightarrow H_{I J}^{(1)},
\end{aligned}
$$

while the quantities related to matter transform as

$$
\begin{aligned}
& \rho^{(1)} \rightarrow \rho^{(1)}+\bar{\rho}^{\prime} T^{(1)} \\
& P^{(1)} \rightarrow P^{(1)}+\bar{P}^{\prime} T^{(1)} \\
& V^{(1)} \rightarrow V^{(1)}-L^{\prime(1)} \\
& \pi^{I J(1)} \rightarrow \pi^{I J(1)},
\end{aligned}
$$

where a prime denotes a derivative w.r.t. to conformal time $\eta$, and where $\mathcal{H} \equiv a^{\prime} / a$. From now on, we shall refer to these first-order transformation rules defined by $\xi_{1}$ as $\mathcal{T}_{\xi_{1}}\left(\Phi^{(1)}\right), \mathcal{T}_{\xi_{1}}\left(B^{(1)}\right), \ldots$ or simply $\mathcal{T}\left(\Phi^{(1)}\right), \mathcal{T}\left(B^{(1)}\right), \ldots$ For instance $\mathcal{T}\left(\Phi^{(1)}\right)=$ $\Phi^{(1)}+T^{\prime(1)}+\mathcal{H} T^{(1)}$.

We first note that the first-order tensorial modes and the first-order anisotropic stress are automatically gauge invariant. We can define gauge invariant variables, by transforming $\Phi^{(1)}$ and $\Psi^{(1)}$ towards the Newtonian gauge (NG) [10. This transformation is defined by the vector field $\xi_{\rightarrow \mathrm{NG}}^{(1)}$ decomposed in $T_{\rightarrow \mathrm{NG}}^{(1)}=B^{(1)}-$ $E^{\prime(1)}, L_{\rightarrow \mathrm{NG}}^{(1)}=-E^{(1)}$, and it transforms the perturbation variables as

$B^{(1)} \rightarrow 0$

$E^{(1)} \rightarrow 0$

$\Phi^{(1)} \rightarrow \hat{\Phi}^{(1)} \equiv{ }_{\mathrm{NG}} \Phi^{(1)}=\Phi^{(1)}+\mathcal{H}\left(B^{(1)}-E^{\prime(1)}\right)+\left(B^{(1)}-E^{(1)}\right)^{\prime}$

$\Psi^{(1)} \rightarrow \hat{\Psi}^{(1)} \equiv{ }_{\mathrm{NG}} \Psi^{(1)}=\Psi^{(1)}-\mathcal{H}\left(B^{(1)}-E^{\prime(1)}\right)$. 
Similarly the gauge-invariant variables that would reduce to $\delta \rho, \delta P$ and $v$ are

$$
\begin{aligned}
& \hat{\rho}^{(1)} \equiv{ }_{\mathrm{NG}} \rho^{(1)}=\rho^{(1)}+\bar{\rho}^{\prime}\left(B^{(1)}-E^{\prime(1)}\right) \\
& \delta^{(1)} \hat{P} \equiv{ }_{\mathrm{NG}} P^{(1)}=P^{(1)}+\bar{P}^{\prime}\left(B^{(1)}-E^{\prime(1)}\right) \\
& \hat{V}^{(1)} \equiv{ }_{\mathrm{NG}} V^{(1)}=V^{(1)}+E^{\prime(1)} \\
& \hat{\pi}^{I J(1)} \equiv{ }_{\mathrm{NG}} \pi^{I J(1)}=\pi^{I J(1)} .
\end{aligned}
$$

Since we have ignored the vector gauge degrees of freedom, $B^{(1)}$ and $E^{(1)}$ are the two gauge variant variables of the metric perturbation while $\hat{\Phi}^{(1)}$ and $\hat{\Psi}^{(1)}$ are the gauge-invariant part. As mentionned before, we then force the gauge-invariant variables in the perturbed metric by replacing $\Phi^{(1)}$ with $\hat{\Phi}^{(1)}-\mathcal{H}\left(B^{(1)}-E^{\prime(1)}\right)+$ $\left(B^{(1)}-E^{\prime(1)}\right)^{\prime}$ and applying similar procedures for $\Psi^{(1)}, \rho^{(1)}$ and $P^{(1)}$. When developping Einstein equations, we know that general covariance will eventually keep only gauge-invariant terms. Thus, we can either do a full calculation and witness the terms involving the degrees of freedom $B^{(1)}$ and $E^{(1)}$ disappear, or perform the calculations with $B^{(1)}$ and $E^{(1)}$ set to zero and obtain the perturbed Einstein equations only in function of gauge-invariant variables. The latter simplifies the computation, which is useful when going to higher orders. This procedure means that we decompose the perturbed metric in a gauge-invariant part and a gauge variant part as

$$
g^{(1)} \equiv \hat{g}^{(1)}+\mathcal{L}_{-\xi_{\rightarrow \mathrm{NG}}^{(1)}} \bar{g}
$$

as it can be seen from the transformation rules under a gauge change characterised by $\xi_{1}$

$$
\begin{aligned}
& \hat{g}^{(1)} \rightarrow \hat{g}^{(1)}, \\
& -\xi_{\rightarrow \mathrm{NG}}^{(1)} \rightarrow-\xi_{\rightarrow \mathrm{NG}}^{(1)}+\xi_{1},
\end{aligned}
$$

and that eventually, only $\hat{g}^{(1)}$ will appear in the equations. This property which is not general but happens to hold in the case of cosmological perturbation (i.e. around FL metric) is the key to extend this construction to second order.

It should be noted that this procedure, although achieved by defining gauge invariant variables which reduce to the perturbation variables in the Newtonian gauge, can be extended to other types of gauge-invariant variables which reduce to perturbation variables in another gauge. 


\subsection{Second-order gauge-invariant variables}

For second-order perturbations, equation (2.14) gives the following transformation rules

$$
\begin{aligned}
& \Phi^{(2)} \rightarrow \Phi^{(2)}+T^{\prime(2)}+\mathcal{H} T^{(2)}+S_{\Phi} \\
& B^{(2)} \rightarrow B^{(2)}-T^{(2)}+L^{\prime(2)}+S_{B} \\
& \Psi^{(2)} \rightarrow \Psi^{(2)}-\mathcal{H} T^{(2)}+S_{\Psi} \\
& E^{(2)} \rightarrow E^{(2)}+L^{(2)}+S_{E} \\
& B_{I}^{(2)} \rightarrow B_{I}^{(2)}+L_{I}^{(2)}+S_{B_{I}} \\
& E_{I}^{(2)} \rightarrow E_{I}^{(2)}+L_{I}^{(2)}+S_{E_{I}} \\
& H_{I J}^{(2)} \rightarrow H_{I J}^{(2)}+S_{H_{I J}} \\
& \rho^{(2)} \rightarrow \rho^{(2)}+\bar{\rho}^{\prime} T^{(2)}+S_{\rho} \\
& P^{(2)} \rightarrow P^{(2)}+\bar{P}^{\prime} T^{(2)}+S_{P} \\
& V^{(2)} \rightarrow V^{(2)}-L^{\prime(2)}+S_{V} \\
& \tilde{V}^{I(2)} \rightarrow \tilde{V}^{I(2)}-L^{\prime(2)}+S_{\tilde{V}^{I}} \\
& \pi^{I J(2)} \rightarrow \pi^{I J(2)}+S_{\pi^{I J}}
\end{aligned}
$$

where the source terms are quadratic in the first-order gauge transformation variables $T^{(1)}, L^{(1)}$, and the first order metric perturbations $\Phi^{(1)}, \Psi^{(1)}, B^{(1)}, E^{(1)}$ and $E_{I J}^{(1)}$. We collect the expressions of these terms in Appendix B. In the rest of this paper, we shall refer to these second-order transformation rules associated with $(\xi) \equiv\left(\xi_{1}, \xi_{2}\right)$ as $\mathcal{T}_{(\xi)}\left(\Phi^{(2)}\right), \mathcal{T}_{(\xi)}\left(B^{(2)}\right), \ldots$ or simply $\mathcal{T}\left(\Phi^{(2)}\right), \mathcal{T}\left(B^{(2)}\right), \ldots$. These transformation rules are much more complicated than their first-order counterparts. However, the combination defined by $F \equiv g^{(2)}+2 \mathcal{L}_{\xi_{\rightarrow \mathrm{NG}}^{(1)}} g^{(1)}+\mathcal{L}_{\xi_{\rightarrow \mathrm{NG}}^{(1)}}^{2} \bar{g}$ enjoys the simple transformation rule $F \rightarrow F+\mathcal{L}_{\xi_{2}+\left[\xi_{\rightarrow \mathrm{NG}}^{(1)}, \xi_{1}\right]} \bar{g}$ under a gauge change defined by $\xi_{2}$ and $\xi_{1}$ (see [43]). As a result, its transformation rule mimics the one of first-order pertubations under a gauge change. This means that if we decompose $F$ in the same way as we did for the metric with

$$
\begin{aligned}
& \Phi_{F} \equiv \Phi^{(2)}+S_{\Phi}\left(\xi_{\rightarrow \mathrm{NG}}^{(1)}\right) \\
& \Psi_{F} \equiv \Psi^{(2)}+S_{\Psi}\left(\xi_{\rightarrow \mathrm{NG}}^{(1)}\right) \\
& B_{F} \equiv B^{(2)}+S_{B}\left(\xi_{\rightarrow \mathrm{NG}}^{(1)}\right) \\
& E_{F} \equiv E^{(2)}+S_{E}\left(\xi_{\rightarrow \mathrm{NG}}^{(1)}\right) \\
& B_{F_{I}} \equiv B_{I}^{(2)}+S_{B_{I}}\left(\xi_{\rightarrow \mathrm{NG}}^{(1)}\right) \\
& E_{F_{I}} \equiv E_{I}^{(2)}+S_{E_{I}}\left(\xi_{\rightarrow \mathrm{NG}}^{(1)}\right) \\
& H_{F_{I J}} \equiv H_{I J}^{(2)}+S_{H_{I J}}\left(\xi_{\rightarrow \mathrm{NG}}^{(1)}\right),
\end{aligned}
$$

then the transformation rules for these quantities will be similar to those of equation (2.24), but with the vector $\xi_{2}+\left[\xi_{\rightarrow \mathrm{NG}}, \xi_{1}\right]$ instead of $\xi_{1}$. Consequently, we shall use the same combinations (taking into account the vector contribution at second order since it is does not vanish at this order) in order to construct gauge-invariant 
variables which are

$$
\begin{aligned}
& \hat{\Phi}^{(2)} \equiv \Phi_{F}+\left(B_{F}-E_{F}^{\prime}\right)^{\prime}+\mathcal{H}\left(B_{F}-E_{F}^{\prime}\right) \\
& \hat{\Psi}^{(2)} \equiv \Psi_{F}-\mathcal{H}\left(B_{F}-E_{F}^{\prime}\right) \\
& \hat{\Phi}_{I}^{(2)} \equiv B_{F_{I}}-E_{F_{I}}^{\prime} \\
& \hat{H}_{I J}^{(2)} \equiv H_{F_{I J}} .
\end{aligned}
$$

This procedure is equivalent to transforming quantities in the Newtonian gauge since it transforms $B, E$ and $E^{I}$ into a null value up to second order. This transformation is defined by $\xi_{\rightarrow \mathrm{NG}}^{(2)}$ that we decompose in

$$
\begin{aligned}
& T_{\rightarrow \mathrm{NG}}^{(2)}=B^{(2)}-E^{\prime(2)}+S_{B}\left(\xi_{\rightarrow \mathrm{NG}}^{(1)}\right)-S_{E}^{\prime}\left(\xi_{\rightarrow \mathrm{NG}}^{(1)}\right) \\
& L_{\rightarrow \mathrm{NG}}^{(2)}=-E^{(2)}-S_{E}\left(\xi_{\rightarrow \mathrm{NG}}^{(1)}\right) \\
& L_{\rightarrow \mathrm{NG}}^{I(2)}=-E^{I(2)}-S_{E^{I}}\left(\xi_{\rightarrow \mathrm{NG}}^{(1)}\right) .
\end{aligned}
$$

The second-order gauge-invariant variables can thus also be defined by

$$
\begin{aligned}
& \hat{\Phi}^{(2)} \equiv{ }_{\mathrm{NG}} \Phi^{(2)} \\
& \hat{\Psi}^{(2)} \equiv{ }_{\mathrm{NG}} \Psi^{(2)} \\
& \hat{\Phi}_{I}^{(2)} \equiv{ }_{\mathrm{NG}} E_{I}^{(2)} \\
& \hat{H}_{I J}^{(2)} \equiv{ }_{\mathrm{NG}} H_{I J}^{(2)} \\
& \hat{\rho}^{(2)} \equiv_{\mathrm{NG}} \rho^{(2)} \\
& \hat{P}^{(2)} \equiv{ }_{\mathrm{NG}} P^{(2)} \\
& \hat{V}^{(2)} \equiv{ }_{\mathrm{NG}} V^{(2)} \\
& \hat{\tilde{V}}^{I(2)} \equiv_{\mathrm{NG}} \tilde{V}^{I(2)} \\
& \hat{\pi}^{I J(2)} \equiv{ }_{\mathrm{NG}} \pi^{I J(2)} .
\end{aligned}
$$

where the index NG indicates that we transformed the quantity with the formula (2.14), with the vector fields $\xi_{\rightarrow \mathrm{NG}}^{(1)}$ and $\xi_{\rightarrow \mathrm{NG}}^{(2)}$ defined above. This procedure means that we have split the second-order metric according to

$$
g^{(2)}=\tilde{g}^{(2)}+\mathcal{L}_{-\xi_{\rightarrow \mathrm{NG}}^{(2)}} \bar{g}+2 \mathcal{L}_{-\xi_{\rightarrow \mathrm{NG}}^{(1)}} g^{(1)}-\mathcal{L}_{-\xi_{\rightarrow \mathrm{NG}}^{(1)}} \bar{g},
$$

where $\tilde{g}^{(2)}$ is the gauge-invariant part and $-\xi_{\rightarrow \mathrm{NG}}^{(2)}$ the gauge variant part, as it can be seen from the transformation rules under a gauge change characterised by $\left(\xi_{1}, \xi_{2}\right)$

$$
\begin{aligned}
& \tilde{g}^{(2)} \rightarrow \tilde{g}^{(2)} \\
& -\xi_{\rightarrow \mathrm{NG}}^{(2)} \rightarrow-\xi_{\rightarrow \mathrm{NG}}^{(2)}+\xi_{2}+\left[\xi_{\rightarrow \mathrm{NG}}^{(1)}, \xi_{1}\right] .
\end{aligned}
$$

\section{Gauge transformations and gauge invariance for a distribution function}

\section{1. pre-Riemannian distribution function in the unpolarized case}

So far, we have set up the mathematical framework to identify points between the background space-time and the perturbed space-times through a gauge field $X$. This enabled us to define the perturbation of tensors and to calculate their transformation properties under a gauge transformation. However this allows only to perform a fluid 
treatment of the radiation. In the statistical description for a set of particles, we assume that each particle has a given impulsion $p^{\mu}$ and is located at a given position [76. The equations then have to describe the phase space distribution of the particles. If the number of particles is high enough, we can define a probability density, the one-particle distribution function, of finding a particle in an infinitesimal volume of the phase space. Now, let us focus our attention on this distribution function. The distribution function is a function of the point considered (i.e. its coordinates $x^{A}$ ), and also a function of the tangent space at this point whose coordinate we label by $p^{\nu} \partial_{\nu}$. There is no special reason for this function to be linear in $p^{\nu} \partial_{\nu}$, but we can expand it, without any loss of generality, in power series of tensors according to

$$
f\left(x^{A}, p^{\nu}\right)=\sum_{k} F_{\mu_{1} \ldots \mu_{k}}\left(x^{A}\right) p^{\mu_{1}} \ldots p^{\mu_{k}} .
$$

Here $f$ stands for either the intensity $I$, the degree of circular polarization $V$ or the matter distribution functions $g_{\mathrm{e}}, g_{\mathrm{b}}$ and $g_{\mathrm{c}}$. From the previous section we know the transformation rules for the tensorial quantities $F_{\mu_{1} . . \mu_{k}}$, thus $f$ transforms according to

$$
\mathcal{T}_{(\xi)}\left[f\left(x^{A}, p^{\nu}\right)\right] \equiv \sum_{k} \mathcal{T}_{(\xi)}\left[F_{\mu_{1} . . \mu_{k}}\left(x^{A}\right)\right] p^{\mu_{1}} \ldots p^{\mu_{k}},
$$

where $\mathcal{T}_{(\xi)}$ refers to the knight-diffeomorphism with the set of vectors $\left(\xi_{1}, \xi_{2}, \ldots\right)$.

As we do not necessarily want to refer explicitly to the decomposition in multipoles, we use the fact that for any vector $\xi=\xi^{\mu} \partial_{\mu}$, which defines a flow on the background space-time $\mathcal{P}_{0}(\mathcal{N})$, we can define an induced flow (a natural lift) on the vector tangent bundle $T \mathcal{P}_{0}(\mathcal{N})$ directed by the vector field $T \xi=\left[\xi^{\mu} \partial_{\mu}, p^{\nu}\left(\partial_{\nu} \xi^{\mu}\right) \frac{\partial}{\partial p^{\mu}}\right]$. This implies the useful property

$$
\mathcal{L}_{\xi}\left(F_{\mu_{1} . . \mu_{p}}\right) p^{\mu_{1}} . . p^{\mu_{p}}=\mathcal{L}_{T \xi}\left(F_{\mu_{1} . . \mu_{p}} p^{\mu_{1}} . . p^{\mu_{p}}\right) .
$$

With this definition, we can rewrite the transformation rule for $f$ as

$$
\mathcal{T}_{(\xi)}\left[f\left(x^{A}, p^{\nu}\right)\right]=\mathcal{T}_{(T \xi)}\left[f\left(x^{A}, p^{\nu}\right)\right],
$$

where now $\mathcal{T}_{(T \xi)}$ refers to the knight-diffeomorphism with the set of vectors $\left(T \xi_{1}, T \xi_{2}, \ldots\right)$. The evolution of the distribution function is dictated by the Boltzmann equation, and its collision term can be expressed in the local Minkowskian frame defined by a tetrad fields $\mathbf{e}_{a}$, from known particles physics. For this reason, the framework developed to define gauge transformations for a general manifold has to be extended to the case of Riemannian manifold. Instead of using the coordinates basis $\partial_{A}$ to express a vector of tangent space as $\mathbf{p}=p^{A} \partial_{A}$, we prefer to use the tetrads basis $\mathbf{e}_{a}$ and write $\mathbf{p}=p^{a} \mathbf{e}_{a}$. In terms of coordinates, this means that the distribution function is a function of $x^{A}$ and $p^{a}$, and that we favor the expansion (1.28) instead of the expansion (3.1). When expressing the physics with the tetrad fields, the metric is not just one of the many tensors of the theory whose properties under a gauge transformation we need to know, but rather a central feature of the manifold, since it determinates the tetrads (up to a Lorentz tranformation) required to express the distribution function. 


\subsection{Perturbation of tetrads}

3.2.1. Pulling back the tetrad With the formalism developed for tensors, we carry this tetrad field onto the background space-time using a gauge field $X$ with

$$
\begin{aligned}
& X \mathbf{e}_{a} \equiv \phi_{\lambda, X}^{\star}\left(\mathbf{e}_{a}\right)=\sum_{k=0}^{k=\infty} \frac{\lambda^{k}}{k !} \mathcal{L}_{X}^{k} \mathbf{e}_{a} \\
& \left.{ }_{X} \mathbf{e}_{a}^{(n)} \equiv \mathcal{L}_{X}^{n} \mathbf{e}_{a}\right|_{\mathcal{P}_{0}(\mathcal{N})} \quad \overline{\mathbf{e}}_{a} \equiv{ }_{X} \mathbf{e}_{a}^{(0)},
\end{aligned}
$$

and similar formulas for $\mathbf{e}^{a}$.

As $\overline{\mathbf{e}}_{a}$ is a basis of the tangent space on the background space-time (and $\overline{\mathbf{e}}^{a}$ a basis of its dual space), ${ }_{X} \mathbf{e}_{a}$ and ${ }_{X} \mathbf{e}^{a}$ which also lie on the background space-time can be expressed in the generic form

${ }_{X} \mathbf{e}_{a}={ }_{X} R_{a}^{b} \overline{\mathbf{e}}_{b}, \quad{ }_{X} \mathbf{e}^{b}=\overline{\mathbf{e}}^{a}{ }_{X} S_{a}^{b}, \quad{ }_{X} R_{a}^{c}{ }_{X} S_{c}^{b}={ }_{X} S_{a}^{c}{ }_{X} R_{c}^{b}=\delta_{a}^{b}$,

where

$$
{ }_{X} R_{a b} \equiv \sum_{k} \frac{\lambda^{k}}{k !}{ }_{X} R_{a b}^{(k)}, \quad{ }_{X} S_{a b} \equiv \sum_{k} \frac{\lambda^{k}}{k !}{ }_{x} S_{a b}^{(k)} .
$$

Order by order, this reads

$$
{ }_{X} \mathbf{e}_{a}^{(n)}={ }_{X} R_{a}^{(n) b} \overline{\mathbf{e}}_{b}, \quad{ }_{X} \mathbf{e}^{b(n)}=\overline{\mathbf{e}}^{a}{ }_{X} S_{a}^{(n) b} .
$$

3.2.2. Normalization condition Tetrads are four vector fields which satisfy equation (1.1) and are thus related to the metric. Consequently, the perturbations of the tetrad defined above are partly related to the perturbations of the metric. When pulled back to the background space-time, equation (1.1) implies

$$
\begin{aligned}
\phi_{\lambda, X}^{\star}\left(\eta_{a b}\right)=\eta_{a b} & =\phi_{\lambda, X}^{\star}\left(e_{a}^{\mu} e_{b}^{\nu} g_{\mu \nu}\right) \\
& =\phi_{\lambda, X}^{\star}\left(e_{a}^{\mu}\right) \phi_{\lambda, X}^{\star}\left(e_{b}^{\nu}\right) \phi_{\lambda, X}^{\star}\left(g_{\mu \nu}\right) .
\end{aligned}
$$

Identifying order by order we get in particular for the first and second orders

$$
\begin{aligned}
& \overline{\mathbf{e}}_{b \cdot X} \mathbf{e}_{a}^{(1)}+\overline{\mathbf{e}}_{a} \cdot X \mathbf{e}_{b}^{(1)}+{ }_{x} g^{(1)}\left(\overline{\mathbf{e}}_{a}, \overline{\mathbf{e}}_{b}\right)=0, \\
& \overline{\mathbf{e}}_{b \cdot X} \mathbf{e}_{a}^{(2)}+\overline{\mathbf{e}}_{a} \cdot X \mathbf{e}_{b}^{(2)}+{ }_{x} g^{(2)}\left(\overline{\mathbf{e}}_{a}, \overline{\mathbf{e}}_{b}\right)+{ }_{X} \mathbf{e}_{b}^{(1)} \cdot X \mathbf{e}_{a}^{(1)} \\
& +{ }_{x} g^{(1)}\left({ }_{X} \mathbf{e}_{a}^{(1)}, \overline{\mathbf{e}}_{b}\right)+{ }_{x} g^{(1)}\left(\overline{\mathbf{e}}_{a},{ }_{X} \mathbf{e}_{b}^{(1)}\right)=0,
\end{aligned}
$$

where a dot product here stands for $\bar{g}($,$) . From the constraints (3.10), we can$ determine the symmetric part of $R_{a b}^{(n)}$ as

$$
\begin{aligned}
{ }_{X} R_{(a b)}^{(1)}= & -\frac{1}{2}{ }_{x} g^{(1)}\left(\overline{\mathbf{e}}_{a}, \overline{\mathbf{e}}_{b}\right), \\
{ }_{X} R_{(a b)}^{(2)}= & -\frac{1}{2}{ }_{x} g^{(2)}\left(\overline{\mathbf{e}}_{a}, \overline{\mathbf{e}}_{b}\right)-{ }_{x} g^{(1)}\left({ }_{X} R_{a c}^{(1)} \overline{\mathbf{e}}^{c}, \overline{\mathbf{e}}_{b}\right) \\
& -{ }_{x} g^{(1)}\left(\overline{\mathbf{e}}_{a},{ }_{X} R_{b c}^{(1)} \overline{\mathbf{e}}^{c}\right)-{ }_{X} R_{a}^{(1) c}{ }_{X} R_{b c}^{(1)},
\end{aligned}
$$

which are related to the components of the inverse by

$$
\begin{aligned}
& { }_{X} S_{a b}^{(1)}=-{ }_{X} R_{a b}^{(1)}, \\
& { }_{X} S_{a b}^{(2)}=-{ }_{X} R_{a b}^{(2)}+{ }{ }_{X} R_{a}^{(1) c}{ }_{X} R_{c b}^{(1)} .
\end{aligned}
$$


The antisymmetric part, ${ }_{x} R_{[a b]}$, still remains to be chosen as it corresponds to the Lorentz transformation freedom (boost and rotation), which is allowed by the definition (1.1). A first and easy choice would be ${ }_{X} R_{[a b]}^{(n)}=0$ for any $n$. However, as mentioned above, we eventually want to decompose a vector $p^{A} \partial_{A}$ on tangent space as

$$
p^{B} \partial_{B}=p^{a} \mathbf{e}_{a}=p^{a} e_{a}^{B} \partial_{B},
$$

and identify $p^{o}$ with the energy and $p^{i}$ with the momentum (although conserved quantities are generally ill-defined in general relativity, energy and momentum can be defined when performing perturbations around a maximally symmetric background [77. as it is the case here). When working with coordinates, we want to express physical quantities, as measured by a set of fundamental observers that we need to choose. If we choose the velocity of these fundamental observers as orthonormal to the constant time coordinate hypersurfaces, then we require [78] $\mathbf{e}^{o} \sim \mathrm{d} \eta$, which is equivalent to choose ${ }_{x} S_{i o}^{(n)}=0$ for any $n$. This choice allows us to fix the boost in $S^{(n)}$ by imposing the condition ${ }_{X} S_{[i o]}^{(n)}=-{ }_{X} S_{[o i]}^{(n)}=-{ }_{X} S_{(i o)}^{(n)}$, and it can be checked by recursion that this implies

$$
{ }_{X} R_{[i o]}^{(n)}=-{ }_{X} R_{[o i]}^{(n)}=-{ }_{X} R_{(i o)}^{(n)} .
$$

We also fix the rotation by requiring $X S_{[i j]}^{(n)}=0$, and it can be checked similarly, that this implies ${ }_{X} R_{[i j]}^{(n)}=0$.

\subsection{Gauge transformation of tetrads}

Under a gauge transformation, we can deduce the transformation properties of the tetrad from those of the perturbed metric. In the FL case, we use a natural background tetrad associated to Cartesian coordinates

$$
\overline{\mathbf{e}}_{o} \equiv\left(\partial_{O}\right) / a, \quad \overline{\mathbf{e}}_{i} \equiv\left(\partial_{I}\right) / a
$$

in order to evaluate equation (3.11). We report the detailed expressions for the transformation of the tetrads for the first and second orders in Appendix C. Since the tetrads are constrained by equation (3.9), the transformation is necessarily of the form

$$
\mathcal{T}\left(\mathbf{e}_{a}\right)=\Lambda_{a}^{b} \mathcal{T}_{\text {knight }}\left(\mathbf{e}_{b}\right), \quad \mathcal{T}\left(\mathbf{e}^{a}\right)=\Lambda_{b}^{a} \mathcal{T}_{\text {knight }}\left(\mathbf{e}^{b}\right),
$$

where $\mathcal{T}_{\text {knight }}$ refers to the transformation that one would obtain by considering each of the four vectors fields in the tetrad as independent vector fields, whose transformation rule is calculated using the knight diffeomorphism of equations (2.14). As a result of the choice $R_{[i j]}=S_{[i j]}=0$, we get that $\Lambda_{i}^{j}=\delta_{i}^{j}$, and the three vectors $\mathbf{e}_{i}$ in a tetrad transform as independent vector fields. Similarly, if a vector field $\mathbf{W}$ satisfies $\mathbf{W} . \mathbf{e}_{o}=0$, then $W^{i}$ transform as a scalar field under gauge transformations (recall that $\left.W^{i} \equiv \mathbf{e}^{i} . \mathbf{W}\right)$. This property extends of course to higher rank tensors, which means that the tetrad components of a projected tensor are to be considered as scalars under gauge transformations. 


\subsection{Gauge transformation of a distribution function}

3.4.1. The perturbed distribution function with a tetrad Now that the transformation properties of the tetrads are known, we turn to the general transformation of a distribution function $f\left(x^{A}, p^{a}\right)$. First, by using the tetrad we can deduce a multipole decomposition of the type (1.28) from the decomposition (3.1) by

$$
\begin{aligned}
f\left(x^{A}, p^{\mu}\right) & \equiv \sum_{\ell} F_{\mu_{1} \ldots \mu_{\ell}}\left(x^{A}\right) p^{\mu_{1}} \ldots p^{\mu_{\ell}} \\
& =\sum_{\ell}\left[F_{\mu_{1} \ldots \mu_{p}}\left(x^{A}\right) e_{a_{1}}^{\mu_{1}} \ldots e_{a_{\ell}}^{\mu_{\ell}}\right] p^{a_{1}} \ldots p^{a_{\ell}} \\
& \equiv \sum_{\ell} F_{a_{\ell}}\left(x^{A}\right) p \underline{a_{\ell}} \\
& \equiv f\left(x^{A}, p^{a}\right) .
\end{aligned}
$$

Since the momentum is constrained to be on the mass shell, only three of the four components are independent, and we can thus choose the multipoles $F_{a_{\ell}}\left(x^{A}\right)$ to be projected, that is to say if any of their indices is $o$, then they vanish. Note that in the case of radiation, that is for $f \equiv I$, then $F_{a_{\ell}}=\left(p^{o}\right)^{\ell} I_{a_{\ell}}$.

We do not need any additional identification procedure for the tangent spaces through a gauge field, in order to identify points of the tangent space of the slices $T \mathcal{P}_{\lambda}(\mathcal{N})$. Indeed, once the metric and a gauge field $X$ are chosen, there exists a natural identification with the tetrad fields. First, and as mentioned before, we identify the points of $\mathcal{N}$ which lie on the same integral curves of $X$, that is, we identify a point $P \in \mathcal{P}_{0}(\mathcal{N})$ and $\Phi_{\lambda, X}(P) \in \mathcal{P}_{\lambda}(\mathcal{N})$. Then, we identify vectors of their respective tangent spaces, if the coordinates of these vectors in their respective local tetrad frames $\overline{\mathbf{e}}_{a}$ and $\mathbf{e}_{a}$, are the same. To be short, we identify $p^{a} \mathbf{e}_{a}$ and $p^{a} \overline{\mathbf{e}}_{a}$. The multipoles $F_{a_{\ell}}\left(x^{A}\right)$ are scalar fields, and they can be pulled back on the background space-time using the gauge field $X$, and we define in this way perturbations

$$
\Phi_{\lambda, X}^{\star}\left[F_{\underline{a_{\ell}}}\left(x^{A}\right)\right] \equiv{ }_{X} F_{\underline{a_{\ell}}}\left(x^{A}\right) \equiv \sum_{\lambda} \frac{\lambda^{n}}{n !}{ }_{X} F_{\underline{a_{\ell}}}^{(n)}\left(x^{A}\right) .
$$

This perturbation scheme induces a perturbation procedure for the distribution function $f$ as

$$
\begin{aligned}
X f\left(x^{A}, p^{a}\right) & \equiv \sum_{n} \frac{\lambda^{n}}{n !} X f^{(n)}\left(x^{A}, p^{a}\right), \\
X f^{(n)}\left(x^{A}, p^{a}\right) & \equiv \sum_{\ell}{ }_{X} F_{\underline{a_{\ell}}}^{(n)}\left(x^{A}\right) p \underline{a_{\ell}} .
\end{aligned}
$$

It is essential to stress that $p^{a}$ is not a perturbed quantity, it is a coordinate of the locally Minkowskian tangent space. However, the tetrad field allows us to see $\mathbf{p}$ as a perturbed vector since $\mathbf{p}\left(p^{a}\right)=\mathbf{e}_{a} p^{a}$. In other words, for a given $p^{a}$, there is an associated vector whose order by order perturbation in a given gauge $X$ is given by ${ }_{X} \mathbf{p}^{(n)} \equiv{ }_{X} \mathbf{e}_{a}^{(n)} p^{a}$.

3.4.2. Gauge transformation of a distribution function We can deduce the transformation rule under a gauge change directly on the form (3.19), pulled back to the background space-time,

$\mathcal{T}\left[{ }_{X} f\left(x^{A}, p^{a}\right)\right] \equiv \sum_{\ell} \mathcal{T}\left[{ }_{X} \mathcal{F}_{\mu_{1} \ldots \mu_{\ell}}\left(x^{A}\right)\right] \mathcal{T}\left({ }_{X} e_{a_{1}}^{\mu_{1}}\right) \ldots \mathcal{T}\left({ }_{x} e_{a_{\ell}}^{\mu_{\ell}}\right) p^{a_{1}} \ldots p^{a_{\ell}}$ 
The first factor in this expression is tensorial. Exactly as for the pre-Riemannian case, its transformation rule is dictated by the knight-diffeomorphism, whereas we get the transformation rules of the tetrads from equations (C.2) and equations (C.4). As we do not necessarily want to refer explicitly to the multipole expansion, the first factor is rewritten by considering $f$ as a function of $p^{\mu}$ using $p^{a}={ }_{x} e_{\nu}^{a} p^{\nu}$, and applying equation (3.4). We then have to consider the resulting distribution function as a function of $p^{a}$, knowing that the inversion is now given by $\mathbf{p}\left(p^{a}\right)=\mathcal{T}\left(\mathbf{e}_{a}\right) p^{a}$. This will account for $\mathcal{T}\left({ }_{x} e_{a_{1}}^{\mu_{1}}\right) \ldots \mathcal{T}\left({ }_{x} e_{a_{\ell}}^{\mu_{\ell}}\right)$ in equation (3.22). In a compact form it reads

$$
\mathcal{T}\left[{ }_{x} f\left(x^{A}, p^{a}\right)\right]=\left.\mathcal{T}_{(T \xi)}\left\{{ }_{x} f\left[x^{A}, e_{\mu}^{a} p^{\mu}\right]\right\}\right|_{p^{\mu}=\mathcal{T}\left(e_{b}^{\mu}\right) p^{b}} .
$$

To obtain an order by order formula, we explicit these three steps using a Taylor expansion. First, we use that

$$
{ }_{x} f\left(x^{A}, p^{a}\right)=\left[\exp \left(\bar{e}_{\mu}^{b} p^{\mu}{ }_{X} S_{b}{ }^{a} \frac{\partial}{\partial p^{a}}\right){ }_{x} f\right]\left(x^{A}, \bar{e}_{\mu}^{b} p^{\mu}\right) \equiv_{x} g\left(x^{A}, p^{\mu}\right),
$$

in order to consider $f$ as a function of $p^{\mu}$. We then Taylor expand again the result of the knight-diffeomorphism in order to read the result as a function of $p^{a}$,

$\left.\mathcal{T}\left[{ }_{x} f\left(x^{A}, p^{a}\right)\right]=\left[\exp \left(\bar{e}_{b}^{\mu} p^{a} \mathcal{T}\left({ }_{x} R_{a}{ }^{b}\right) \frac{\partial}{\partial p^{\mu}}\right) \mathcal{T}_{(T \xi)}{ }_{{ }_{x}} g\right)\right]\left(x^{A}, \bar{e}_{a}^{\mu} p^{a}\right)$.

The derivatives in the previous expressions have to be ordered on the right in each term of the expansion in power series of the exponential. When identifying order by order, we need to take into account the expansion in $R_{a b}$ and $S_{a b}$, in the exponentials and also in the knight-diffeomorphism. The detail of the transformation properties of the first and second-order distribution function can be found in 45. We report here the final result of the transformation rule for the first order and the second order distribution function for radiation

$$
\begin{aligned}
\mathcal{T}\left[{ }_{X} f^{(1)}\right]={ }_{X} f^{(1)}+\frac{\partial \bar{f}}{\partial p^{o}} p^{o} n^{i} \partial_{I} T+T \frac{\partial \bar{f}}{\partial \eta} & \\
\mathcal{T}\left({ }_{x} f^{(2)}\right)= & { }_{X} f^{(2)}+\frac{\partial \bar{f}}{\partial \eta}\left(T^{(2)}+T T^{\prime}+\partial_{I} T \partial^{I} L\right) \\
& +\frac{\partial \bar{f}}{\partial p^{o}}\left\{p^{i} \partial_{I} T^{(2)}-2 p^{j}\left[\left(\partial_{I} \partial_{J} E+E_{I J}+\partial_{I} \partial_{J} L\right) \partial^{I} T-\Psi \partial_{J} T\right]\right. \\
& \left.\quad+p^{o} \partial_{I} T \partial^{I} T+p^{i}\left(T \partial_{I} T\right)^{\prime}+p^{i} \partial_{I}\left(\partial^{J} L \partial_{J} T\right)+2 \Phi p^{i} \partial_{I} T\right\} \\
& +\frac{\partial^{2} \bar{f}}{\partial\left(p^{o}\right)^{2}}\left(p^{i} \partial_{I} T p^{j} \partial_{J} T\right)+2 \frac{\partial^{2} \bar{f}}{\partial \eta \partial p^{o}} T p^{i} \partial_{I} T+\frac{\partial^{2} \bar{f}}{\partial \eta^{2}} T^{2} \\
& +2 \frac{\partial_{X} f^{(1)}}{\partial p^{o}} p^{j} \partial_{J} T+2 \frac{\partial_{X} f^{(1)}}{\partial p^{i}} p^{o} \partial^{I} T+2 \partial^{I} L \partial_{I_{X}} f^{(1)}+2 T \frac{\partial_{X} f^{(1)}}{\partial \eta}
\end{aligned}
$$

In order to derive this formula, no use has been made of the normalization of the momentum. Consequently in the above transformation rule, $f$ stands either for $I, V$ or for $g_{\mathrm{e}}, g_{\mathrm{b}}$ (where we conventionally prefer to use the notation $\mathbf{q}$ instead of $\mathbf{p}$ for the momentum of particles). Note here that the Einstein implicit summation rule still applies on index of tetrad type $(i, j, k \ldots)$ when contracted with indices of coordinate type $(I, J, K \ldots)$. This type of contraction arises from the fact that we have chosen a background tetrad field adapted to the coordinate system, that is with $\bar{e}_{a}^{B} \sim \delta_{a}^{B}$ and $\bar{e}_{B}^{a} \sim \delta_{B}^{a}$. 
3.4.3. Gauge transformation of the linear polarization tensor Now if we want to describe polarized radiation, it is straightforward to generalize this calculation. Indeed it is projected, that is $f_{o i}=f_{i o}=f_{o o}=0$, and thanks to the remark at the end of section 3.3, each of the nine components $f_{i j}$ can be treated like a scalar valued distribution function, and the same property is valid for $P_{i j}$. Furthermore, since for symmetry reasons the radiation is not polarized at the background level, that is $\bar{P}_{i j}=0$, its transformation rule reduces to

$\mathcal{T}\left[{ }_{X} P_{k l}^{(1)}\right]={ }_{X} P_{k l}^{(1)}$,

$$
\begin{aligned}
\mathcal{T}\left({ }_{X} P_{k l}^{(2)}\right)= & { }_{X} P_{k l}^{(2)}+2 \frac{\partial_{X} P_{k l}^{(1)}}{\partial p^{o}} p^{j} \partial_{J} T \\
& +2 \frac{\partial_{X} P_{k l}^{(1)}}{\partial p^{i}} p^{o} \partial^{I} T+2 \partial^{I} L \partial_{I_{X}} P_{k l}^{(1)}+2 T \frac{\partial_{X} P_{k l}^{(1)}}{\partial \eta} .
\end{aligned}
$$

We can then form first-order and second-order gauge invariant intensity function, degree of circular polarization and tensor of linear polarization, by transforming these quantities in the Newtonian gauge

$$
\begin{aligned}
& \hat{I}^{(1)} \equiv_{{ }_{\mathrm{NG}}} I^{(1)} \equiv \mathcal{T}_{\xi_{\rightarrow \mathrm{NG}}^{(1)}}\left({ }_{X} I^{(1)}\right) \\
& \hat{V}^{(1)} \equiv_{\mathrm{NG}} V^{(1)} \equiv \mathcal{T}_{\xi_{\rightarrow \mathrm{NG}}^{(1)}}\left({ }_{X} V^{(1)}\right) \\
& \hat{P}_{i j}^{(1)} \equiv_{{ }_{\mathrm{NG}}} P_{i j} \equiv \mathcal{T}_{\xi_{\rightarrow \mathrm{NG}}^{(1)}}\left({ }_{X} P_{i j}^{(1)}\right), \\
& \hat{I}^{(2)} \equiv_{{ }_{\mathrm{NG}}} I^{(2)} \equiv \mathcal{T}_{\left(\xi_{\rightarrow \mathrm{NG}}^{(1)}, \xi_{\rightarrow \mathrm{NG}}^{(2)}\right)}\left({ }_{X} I^{(2)}\right) \\
& \hat{V}^{(2)} \equiv_{\mathrm{NG}} V^{(2)} \equiv \mathcal{T}_{\left(\xi_{\rightarrow \mathrm{NG}}^{(1)}, \xi_{\rightarrow \mathrm{NG}}^{(2)}\right)}\left({ }_{X} V^{(2)}\right) \\
& \hat{P}_{i j}^{(2)} \equiv_{\mathrm{NG}} P_{i j}^{(2)} \equiv \mathcal{T}_{\left(\xi_{\rightarrow \mathrm{NG}}^{(1)}, \xi_{\rightarrow \mathrm{NG}}^{(2)}\right)}\left({ }_{X} P_{i j}^{(2)}\right) .
\end{aligned}
$$

The energy integrated counterparts $\hat{\mathcal{I}}^{(n)}, \hat{\mathcal{V}}^{(n)}$ and $\hat{\mathcal{P}}_{i j}^{(n)}$ with $n=1,2$, follow from a definition similar to equations (1.16). Since the transformation rule (3.27) is valid also for the electrons and protons (or for baryons which is the sum of these two components) distribution functions, the corresponding gauge invariant distribution functions can be defined in a similar manner and we name them $\hat{g}^{(1)}$ and $\hat{g}^{(2)}$ for the first and second order respectively.

\section{The gauge invariant Liouville equation for radiation}

Now that we have a complete formalism to handle the perturbations of the fluid quantities and of the distribution functions, we will use it to express the perturbations of the dynamical equations satisfied by these quantities. In this section we focus on the perturbative expansion of the Liouville operator and in section 6 we detail the perturbative expansion of the collision term. In both cases we will recover the standard 
first order result and the novelty lies in the second order perturbation.

In practice, we want to express the Boltzmann (or the Liouville equation if relevant) in function of $\eta$ rather than the affine parameter $s$, since we want to perform an integration on coordinates. We should thus multiply equation (1.59) by $\frac{\mathrm{d} s}{\mathrm{~d} \eta}=1 / p^{O}$. However, there is no point multiplying with the full expression of $\frac{\mathrm{d} s}{\mathrm{~d} \eta}$ since when focusing on the $n$-th order of the Boltzmann equation, the perturbations $\left(\frac{\mathrm{d} s}{\mathrm{~d} \eta}\right)^{(k)}$ with $0<k<n$ will multiply the $(n-k)$-th order of the perturbed Boltzmann equation and these terms will thus vanish. This is the reason why we will instead multiply only by $\left(\frac{\mathrm{d} s}{\mathrm{~d} \eta}\right)^{(0)}=\left(1 / p^{O}\right)^{(0)}=a / p^{o}$, and we will use the notation

$$
L^{\#}[] \equiv L[]\left(\frac{\mathrm{d} s}{\mathrm{~d} \eta}\right)^{(0)}, \quad C_{a b}^{\#}[] \equiv C_{a b}\left(\frac{\mathrm{d} s}{\mathrm{~d} \eta}\right)^{(0)} .
$$

In order to compute the Liouville operator, we need to calculate the perturbed expression of $\frac{\mathrm{d} p^{\circ}}{\mathrm{d} \eta}$ and $\frac{\mathrm{d} n^{i}}{\mathrm{~d} \eta}$. They can be obtained from the geodesic equation

$$
\frac{\mathrm{d} p_{a}}{\mathrm{~d} s}+\omega_{b a c} p^{c} p^{b}=0, \quad \text { with } \quad \frac{\mathrm{d} p_{a}}{\mathrm{~d} s} \equiv p^{b} \partial_{b} p_{a} \equiv p^{b} e_{b}{ }^{C} \partial_{C}\left(p_{a}\right),
$$

that we pull back to the background space-time in order to extract order by order equations

$$
\left(\frac{\mathrm{d} p_{a}}{\mathrm{~d} s}\right)^{(n)}=-\omega_{b a c}^{(n)} p^{c} p^{b}, \quad n=0,1,2,
$$

and from the perturbative expansion of $p^{O}$

$$
\left(\frac{\mathrm{d} \eta}{\mathrm{d} s}\right)^{(n)}=\left(p^{O}\right)^{(n)}=p^{a} e_{a}^{(n) O}, \quad n=0,1,2 .
$$

It should be noted that the transformation properties of $L^{\#}[]$ under a local change of frame is not similar to that of $L[]$ due to the factor $1 / p^{o}$ which transforms according to equation (1.39) 58.

The result of the Liouville operator up to second order is of the form

$L\left[\bar{X}, X^{(1)}, X^{(2)}\right]=\bar{L}[\bar{X}]+L^{(1)}\left[\bar{X}, X^{(1)}\right]+\frac{1}{2}\left(L^{(2)}\left[\bar{X}, X^{(2)}\right]+L^{(1)(1)}\left[\bar{X}, X^{(1)}\right]\right)$,

where $X$ stands for either $I, V$ or $P_{a b}$. Similarly to Einstein equations, the second order Boltzmann equation will involve either terms which are linear in purely second order quantities that we gather in $L^{(2)}$ [], or terms quadratic in first order quantities that we gather in $L^{(1)(1)}[]$. The linear dependence in these purely second order quantities is, as usual, the same as for the linear dependence obtained for the first order equation with respect to the first order quantities, and this means $L^{(2)}[]=L^{(1)}[]$. In principle, once presented the expression of $L^{(1)}\left[\bar{X}, X^{(1)}\right]$, we only need to report the expression of $L^{(1)(1)}\left[\bar{X}, X^{(1)}\right]$ to fully express the second order equations. In practice however we will report both since we chose to neglect the first order vector degrees of freedom but we cannot neglect them at second order and they will thus contribute to $L^{(2)}[]$. Additionally, we also choose to neglect the first order tensorial perturbations. Though they are generated in standard models of inflation, their amplitude is expected to be much smaller than first order scalar perturbations, and furthermore they decay once they enter the Hubble radius. Similarly to vector modes, we will not neglect the tensor 
modes at second order and their contribution will be also reported in $L^{(2)}[]$.

Since the expressions in the decomposition (4.5) have a dependence in $\left(p^{o}, n^{i}\right)$, we will also perform a multipolar expansion according to equation (1.28) for the equations on $I$ and $V$ (with multipoles $L[I]_{a_{\ell}}$ and $L[V]_{a_{\ell}}$ ) and according to equation (1.33) for the equation involving $P_{a b}$ (with electric and magnetic type multipoles being respectively $L[E]_{a_{\ell}}$ and $\left.L[B]_{a_{\ell}}\right)$.

We will also define the energy integrated Liouville operator by

$$
\mathcal{L}[] \equiv \frac{4 \pi}{(2 \pi)^{3}} \int L[]\left(p^{o}\right)^{3} \mathrm{~d} p^{o},
$$

and use similar definitions for $L^{\#}[], C_{a b}$ and $C_{a b}^{\#}$, and this will lead to define multipoles for these quantities, with an obvious choice of notation. We recall that since the polarization tensor is a function of the position coordinates $x^{A}$, that we do not write explicitly, and of the momentum components $p^{a}$, then the Liouville operator and the collision tensor have a dependence in the same variables. However, in order to simplify the notation, we will not write the dependence in $p^{a}$ throughout this section, which is dedicated to the Liouville operator for radiation, and throughout section 5 , which is dedicated to the Liouville operator for baryons, but we will restore it in section 6 dedicated to the collision tensor.

\subsection{The background Liouville operator}

At the background level, space is homogeneous and isotropic. Consequently, the radiation is not polarized and its distribution function depends neither on the direction $n^{i}$ of the photon nor on the position in space $x^{I}$. It only depends on $p^{o}$ and $\eta$, which implies that $\frac{\partial \bar{I}}{\partial n^{i}}=\frac{\partial \bar{I}}{\partial x^{T}}=\bar{P}_{a b}=0$. Since the background geodesic equation implies $\left(\frac{\partial p^{o}}{\partial \eta}\right)^{(0)}=-\mathcal{H} p^{o}$, the Liouville operator reads at the background level

$$
\bar{L}^{\#}[\bar{I}]=\frac{\partial \bar{I}}{\partial \eta}-\mathcal{H} p^{o} \frac{\partial \bar{I}}{\partial p^{o}}
$$

where we recall that the derivative w.r.t. $\eta$ is to be taken at $p^{a}$ fixed. Its energy integrated counterpart reads

$$
\overline{\mathcal{L}}^{\#[\overline{\mathcal{I}}]}=\frac{\partial \overline{\mathcal{I}}}{\partial \eta}+4 \mathcal{H} \overline{\mathcal{I}} .
$$

\subsection{The Liouville operator at first order}

The equation required to express the Liouville operator is the first order of equation 4.3 which in the Newtonian gauge leads to

$$
\left(\frac{\mathrm{d} p^{o}}{\mathrm{~d} \eta}\right)^{(1)}=p^{o}\left[-n^{i} \partial_{I} \Phi^{(1)}+\Psi^{(1)^{\prime}}\right] .
$$

and we obtain that the first order perturbed Liouville operator can be expressed in function of gauge invariant quantities by

$L^{\#(1)}\left[\bar{I}, \hat{I}^{(1)}\right]=\frac{\partial \hat{I}^{(1)}}{\partial \eta}+n^{j} \partial_{J} \hat{I}^{(1)}-\mathcal{H} p^{o} \frac{\partial \hat{I}^{(1)}}{\partial p^{o}}+\left[-n^{j} \partial_{J} \hat{\Phi}^{(1)}+\hat{\Psi}^{(1)^{\prime}}\right] p^{o} \frac{\partial \bar{I}}{\partial p^{o}}$.

Its energy integrated counterpart is thus given by

$\mathcal{L}^{\#(1)}\left[\overline{\mathcal{I}}, \hat{\mathcal{I}}^{(1)}\right]=\frac{\partial \hat{\mathcal{I}}^{(1)}}{\partial \eta}+n^{j} \partial_{J} \hat{\mathcal{I}}^{(1)}+4 \mathcal{H} \hat{\mathcal{I}}^{(1)}+4\left[n^{j} \partial_{J} \hat{\Phi}^{(1)}-\hat{\Psi}^{(1)^{\prime}}\right] \overline{\mathcal{I}}$. 
It will prove more convenient to decompose it in multipoles which are given by

$$
\begin{aligned}
\mathcal{L}^{\#(1)}\left[\overline{\mathcal{I}}, \hat{\mathcal{I}}^{(1)}\right]_{\underline{i_{\ell}}}= & \underline{\hat{\mathcal{I}}}_{\underline{i_{\ell}}}^{\prime(1)}+4 \mathcal{H} \hat{\mathcal{I}}_{\underline{i_{\ell}}}^{(1)}+\frac{\ell+1}{(2 \ell+3)} \partial^{J} \hat{\mathcal{I}}_{j \underline{i_{\ell}}}^{(1)}+\partial_{\left\langle I_{\ell}\right.} \underline{\hat{\mathcal{I}}_{i_{\ell-1}}^{(1)}} \\
& +4 \delta_{\ell}^{1} \overline{\mathcal{I}} \partial_{I_{1}} \hat{\Phi}^{(1)}-4 \delta_{\ell}^{0} \overline{\mathcal{I}} \hat{\Psi}^{\prime}(1) .
\end{aligned}
$$

As for $V$, it follows the same equation as $I$ but we will see in section 6 that it is not excited by the collisions and remains null.

Following the same method for the linear polarization tensor leads to

$$
\begin{aligned}
& L^{\#(1)}\left[\hat{P}_{c d}^{(1)}\right]=S_{c}^{a} S_{d}^{b}\left[\frac{\partial \hat{P}_{a b}^{(1)}}{\partial \eta}+n^{j} \partial_{J} \hat{P}_{a b}^{(1)}-\mathcal{H} p^{o} \frac{\partial \hat{P}_{a b}^{(1)}}{\partial p^{o}}\right], \\
& \mathcal{L}^{\#(1)}\left[\hat{\mathcal{P}}_{c d}^{(1)}\right]=S_{c}^{a} S_{d}^{b}\left[\frac{\partial \hat{\mathcal{P}}_{a b}^{(1)}}{\partial \eta}+n^{j} \partial_{J} \hat{\mathcal{P}}_{a b}^{(1)}+4 \mathcal{H} \hat{\mathcal{P}}_{a b}^{(1)}\right] .
\end{aligned}
$$

We can extract the electric and magnetic type multipoles of the energy integrated Liouville operator, and they read

$$
\begin{aligned}
& \mathcal{L}^{\#(1)}\left[\hat{\mathcal{E}}^{(1)}\right]_{\underline{i_{\ell}}}=\hat{\mathcal{E}}_{\underline{i_{\ell}}}^{(1)}+4 \mathcal{H} \hat{\mathcal{E}}_{\underline{i_{\ell}}}^{(1)}+\frac{(\ell-1)(\ell+3)}{(\ell+1)(2 \ell+3)} \partial^{J} \hat{\mathcal{E}}_{\underline{j \underline{\ell}}_{\underline{\ell}}}^{(1)}+\partial_{\left\langle I_{\ell}\right.} \hat{\mathcal{E}}_{\underline{\left.i_{\ell-1}\right\rangle}}^{(1)} \\
& -\frac{2}{(\ell+1)}\left(\operatorname{curl} \hat{\mathcal{B}}^{(1)}\right)_{\underline{i_{\ell}}} \\
& \mathcal{L}^{\#(1)}\left[\hat{\mathcal{B}}^{(1)}\right]_{\underline{i_{\ell}}}=\hat{\mathcal{B}}_{\underline{i_{\underline{\ell}}}}^{\prime(1)}+4 \mathcal{H} \hat{\mathcal{B}}_{\underline{i_{\underline{\ell}}}}^{(1)}+\frac{(\ell-1)(\ell+3)}{(\ell+1)(2 \ell+3)} \partial^{J} \hat{\mathcal{B}}_{j_{\underline{j}}}^{(1)}+\partial_{\left\langle I_{\ell}\right.} \hat{\mathcal{B}}_{\underline{\left.i_{\ell-1}\right\rangle}}^{(1)} \\
& +\frac{2}{(\ell+1)}\left(\operatorname{curl} \hat{\mathcal{E}}^{(1)}\right)_{\underline{i_{\ell}}},
\end{aligned}
$$

where

$$
(\operatorname{curl} X)_{\underline{i_{\ell}}} \equiv \epsilon_{j k\left\langle i_{\ell}\right.} \partial^{J} X_{\left.\underline{i_{\ell-1}}\right\rangle}{ }^{k} .
$$

However, it can be shown that when the first order tensor and vector modes are neglected, which is our case in this paper, the first order magnetic type multipoles are not excited and remain null [15. We will thus discard them from the computation in the following of this paper in order to simplify the intricate expressions of second order perturbations. In order to be consistent with equation (4.16) we will also have to neglect $\left(\operatorname{curl} \hat{\mathcal{E}}^{(1)}\right)_{\underline{i_{\ell}}}$.

\subsection{The Liouville operator at second order}

Following the same method as for the first order case, we obtain the second order evolution of the energy $p^{o}$ which in the Newtonian gauge reads

$$
\begin{gathered}
\left(\frac{\mathrm{d} p^{o}}{\mathrm{~d} \eta}\right)^{(2)}=p^{o}\left[-n^{i} \partial_{I} \Phi^{(2)}+\Psi^{(2)^{\prime}}+\left(\partial_{I} B_{J}^{(2)}-H_{I J}^{(2)}\right) n^{i} n^{j}\right. \\
\left.+2(\Phi-\Psi) n^{i} \partial_{I} \Phi+4 \Psi \Psi^{\prime}\right]
\end{gathered}
$$

We also need the first order photon trajectory which in the Newtonian gauge reads

$$
\left(\frac{\mathrm{d} x^{I}}{\mathrm{~d} \eta}\right)^{(1)}=\left(\frac{p^{I}}{p^{O}}\right)^{(1)}=n^{i}(\Phi+\Psi),
$$


and the first order lensing equation which gives the evolution of the direction $n^{i}$

$$
\left(\frac{\mathrm{d} n^{i}}{\mathrm{~d} \eta}\right)^{(1)}=-S^{i j} \partial_{J}(\Psi+\Phi) .
$$

We then obtain the gauge invariant second order Liouville operator

$$
\begin{aligned}
& L^{\#(2)}\left[\bar{I}, \hat{I}^{(2)}\right]=\frac{\partial \hat{I}^{(2)}}{\partial \eta}+n^{j} \partial_{J} \hat{I}^{(2)}-\mathcal{H} p^{o} \frac{\partial \hat{I}^{(2)}}{\partial p^{o}} \\
& +\left[-n^{j} \partial_{J} \hat{\Phi}^{(2)}+\hat{\Psi}^{(2)^{\prime}}+\left(\partial_{I} \hat{\Phi}_{J}^{(2)}-\hat{H}_{I J}^{(2)^{\prime}}\right) n^{i} n^{j}\right] p^{o} \frac{\partial \bar{I}}{\partial p^{o}}, \\
& L^{\#(1)(1)}\left[\bar{I}, \hat{I}^{(1)}\right]=2(\hat{\Phi}+\hat{\Psi}) n^{i} \partial_{I} \hat{I}^{(1)}-2 S^{i j}\left(\partial_{J} \hat{\Psi}+\partial_{J} \hat{\Phi}\right) \frac{\partial \hat{I}^{(1)}}{\partial n^{i}} \\
& +2 \frac{\partial \hat{I}^{(1)}}{\partial p^{o}} p^{o}\left[-n^{i} \partial_{I} \hat{\Phi}+\hat{\Psi}^{\prime}\right]+\left[2(\hat{\Phi}-\hat{\Psi}) n^{i} \partial_{I} \hat{\Phi}+4 \hat{\Psi} \hat{\Psi}^{\prime}\right] p^{o} \frac{\partial \bar{I}}{\partial p^{o}} \\
& -2 \hat{\Phi} L^{\#(1)}\left[\bar{I}, \hat{I}^{(1)}\right] \text {. }
\end{aligned}
$$

Its energy integrated version is

$$
\begin{aligned}
\mathcal{L}^{\#(2)}\left[\overline{\mathcal{I}}, \hat{\mathcal{I}}^{(2)}\right]= & \frac{\partial \hat{\mathcal{I}}^{(2)}}{\partial \eta}+n^{j} \partial_{J} \hat{\mathcal{I}}^{(2)}+4 \mathcal{H} \hat{\mathcal{I}}^{(2)} \\
& -4 \overline{\mathcal{I}}\left[-n^{j} \partial_{J} \hat{\Phi}^{(2)}+\hat{\Psi}^{(2)^{\prime}}+\left(\partial_{I} \hat{\Phi}_{J}^{(2)}-\hat{H}_{I J}^{(2)^{\prime}}\right) n^{i} n^{j}\right], \\
\mathcal{L}^{\#(1)(1)}\left[\overline{\mathcal{I}}, \hat{\mathcal{I}}^{(1)}\right]= & 2(\hat{\Phi}+\hat{\Psi}) n^{i} \partial_{I} \hat{\mathcal{I}}^{(1)}-2 S^{i j}\left(\partial_{J} \hat{\Psi}+\partial_{J} \hat{\Phi}\right) \frac{\partial \hat{\mathcal{I}}^{(1)}}{\partial n^{i}} \\
& -8 \hat{\mathcal{I}}^{(1)}\left[-n^{i} \partial_{I} \hat{\Phi}+\hat{\Psi}^{\prime}\right]-4 \overline{\mathcal{I}}\left[2(\hat{\Phi}-\hat{\Psi}) n^{i} \partial_{I} \hat{\Phi}+4 \hat{\Psi} \hat{\Psi}^{\prime}\right] \\
& -2 \hat{\Phi} \mathcal{L}^{\#(1)}\left[\overline{\mathcal{I}}, \hat{\mathcal{I}}^{(1)}\right] .
\end{aligned}
$$

We recall again that we have omitted some exponents stating that some quantities are first order perturbations variables, since at second order, the terms involved are either involving purely second order perturbations or terms quadratic in first order quantities, and there is thus never any ambiguity. Note also that, if $L^{\#}[]$ and $\mathcal{L}^{\#}[]$ had been defined with $(\mathrm{d} s / \mathrm{d} \eta)$ instead of $(\mathrm{d} s / \mathrm{d} \eta)^{(0)}$ in the definition (4.1), the last line of equations (4.22) and (4.24) would not be there, and this enables us to compare with the results of [48, 49] where such different choice has been made (though it is then inconsistent with the collision term of [48] which has been calculated with $(\mathrm{d} s / \mathrm{d} \eta)^{(0)}$, but not with the collision term reported in 49 which seems to be defined with $(\mathrm{d} s / \mathrm{d} \eta)$ though it is stated that it is the same expression as in 48]). We can then extract the multipoles of these equations and we obtain

$$
\begin{aligned}
& \left.\mathcal{L}^{\#(2)}\left[\overline{\mathcal{I}}, \hat{\mathcal{I}}^{(2)}\right]_{\underline{i_{\ell}}}=\hat{\mathcal{I}}_{\underline{i_{\ell}}}^{(2)}+4 \mathcal{H} \hat{\mathcal{I}}_{\underline{i_{\ell}}}^{(2)}+\frac{\ell+1}{(2 \ell+3)} \partial^{J} \hat{\mathcal{I}}_{j_{\underline{i_{\ell}}}}^{(2)}+\partial_{\left\langle I_{\ell}\right.} \hat{\mathcal{I}}_{\underline{i_{\ell-1}}}^{(2)}\right\rangle \\
& -4 \delta_{\ell}^{2} \overline{\mathcal{I}}\left(\partial_{\left(I_{1}\right.} \hat{\Phi}_{\left.I_{2}\right)}^{(2)}-\hat{H}_{I_{1} I_{2}}^{(2)^{\prime}}\right)+4 \delta_{\ell}^{1} \overline{\mathcal{I}} \partial_{I_{1}} \hat{\Phi}^{(2)}-4 \delta_{\ell}^{0} \overline{\mathcal{I}} \hat{\Psi}^{\prime(2)}, \\
& \mathcal{L}^{\#(1)(1)}\left[\hat{\mathcal{I}}^{(1)}\right]_{\underline{i_{\ell}}}=\frac{(\ell+1)}{(2 \ell+3)}\left[8 \partial^{J} \Phi-2(\ell+2) \partial^{J}(\Phi+\Psi)+2 \Psi \partial^{J}\right] \hat{\mathcal{I}}_{j \underline{i_{\ell}}}^{(1)} \\
& +\left[8 \partial_{\left\langle I_{1}\right.} \Phi+2(\ell-1) \partial_{\left\langle I_{1}\right.}(\Phi+\Psi)+2 \Psi \partial_{\left\langle I_{1}\right.}\right] \underline{\hat{\mathcal{I}}_{\left.i_{\ell-1}\right\rangle}^{(1)}} \\
& -8 \Psi^{\prime} \hat{\mathcal{I}}_{\underline{i_{\ell}}}^{(1)}-2 \Phi \hat{\mathcal{I}}_{\underline{i_{\ell}}}^{(1)^{\prime}}-8 \mathcal{H} \Phi \hat{\mathcal{I}}_{i_{\underline{\ell}}}^{(1)} \\
& +4 \delta_{\ell}^{1} \overline{\mathcal{I}}\left[2(\Psi-2 \Phi) \partial_{I} \Phi\right]+4 \delta_{\ell}^{0} \overline{\mathcal{I}}\left[(2 \Phi-4 \Psi) \Psi^{\prime}\right] \text {. }
\end{aligned}
$$


Following the same method as for the intensity part of the Liouville operator, we obtain the Liouville operator for the linear polarization tensor

$$
\begin{aligned}
& L^{\#(2)}\left[\hat{P}_{c d}^{(2)}\right]=S_{c}^{a} S_{d}^{b}\left[\frac{\partial \hat{P}_{a b}^{(2)}}{\partial \eta}+n^{j} \partial_{J} \hat{P}_{a b}^{(2)}-\mathcal{H} p^{o} \frac{\partial \hat{P}_{a b}^{(2)}}{\partial p^{o}}\right], \\
& L^{\#(1)(1)}\left[\hat{P}_{c d}^{(1)}\right]=S_{c}^{a} S_{d}^{b}\left[2(\hat{\Phi}+\hat{\Psi}) n^{i} \partial_{I} \hat{P}_{a b}^{(1)}-2 S^{i j}\left(\partial_{J} \hat{\Psi}+\partial_{J} \hat{\Phi}\right) \frac{\partial \hat{P}_{a b}^{(1)}}{\partial n^{i}}\right. \\
& \left.+2 \frac{\partial \hat{P}_{a b}^{(1)}}{\partial p^{o}} p^{o}\left(-n^{i} \partial_{I} \hat{\Phi}+\hat{\Psi}^{\prime}\right)-2 \hat{\Phi} L^{\#(1)}\left[\hat{P}_{a b}^{(1)}\right]\right],
\end{aligned}
$$

and its energy integrated counterpart

$$
\begin{aligned}
& \mathcal{L}^{\#(2)}\left[\hat{\mathcal{P}}_{c d}^{(2)}\right]=S_{c}^{a} S_{d}^{b}\left[\frac{\partial \hat{\mathcal{P}}_{a b}^{(2)}}{\partial \eta}+n^{j} \partial_{J} \hat{\mathcal{P}}_{a b}^{(2)}+4 \mathcal{H} \hat{\mathcal{P}}_{a b}^{(2)}\right] \\
& \mathcal{L}^{\#(1)(1)}\left[\hat{\mathcal{P}}_{c d}^{(1)}\right]=S_{c}^{a} S_{d}^{b}[ {\left[2(\hat{\Phi}+\hat{\Psi}) n^{i} \partial_{I} \hat{\mathcal{P}}_{a b}^{(1)}-2 S^{i j}\left(\partial_{J} \hat{\Psi}+\partial_{J} \hat{\Phi}\right) \frac{\partial \hat{\mathcal{P}}_{a b}^{(1)}}{\partial n^{i}}\right.} \\
&\left.-8 \hat{\mathcal{P}}_{a b}^{(1)}\left(-n^{i} \partial_{I} \hat{\Phi}+\hat{\Psi}^{\prime}\right)-2 \hat{\Phi} \mathcal{L}^{\#(1)}\left[\hat{\mathcal{P}}_{a b}^{(1)}\right]\right] .
\end{aligned}
$$

We extract the electric and magnetic type multipoles

$$
\begin{aligned}
& \left.\mathcal{L}^{\#(2)}\left[\hat{\mathcal{E}}^{(2)}\right]_{\underline{i_{\ell}}}=\hat{\mathcal{E}}_{\underline{i_{\ell}}}^{(2)}+4 \mathcal{H} \hat{\mathcal{E}}_{\underline{i_{\underline{\ell}}}}^{(2)}+\frac{(\ell-1)(\ell+3)}{(\ell+1)(2 \ell+3)} \partial^{J} \hat{\mathcal{E}}_{\underline{j} \underline{i_{\ell}}}^{(2)}+\partial_{\left\langle I_{\ell}\right.} \hat{\mathcal{E}}_{\underline{i_{\ell-1}}}^{(2)}\right\rangle \\
& -\frac{2}{(\ell+1)}\left(\operatorname{curl} \hat{\mathcal{B}}^{(2)}\right)_{\underline{i_{\ell}}} \text {, } \\
& \mathcal{L}^{\#(1)(1)}\left[\hat{\mathcal{E}}^{(1)}\right]_{\underline{i_{\ell}}}=\frac{(\ell-1)(\ell+3)}{(2 \ell+3)(\ell+1)}\left[8 \partial^{J} \hat{\Phi}-2(\ell+2) \partial^{J}(\hat{\Phi}+\hat{\Psi})+2 \hat{\Psi} \partial^{J}\right] \hat{\mathcal{E}}_{j \underline{i_{\ell}}} \\
& +\left[8 \partial_{\left\langle I_{\ell}\right.} \hat{\Phi}+2(\ell-1) \partial_{\left\langle I_{\ell}\right.}(\hat{\Phi}+\hat{\Psi})+2 \hat{\Psi} \partial_{\left\langle I_{\ell}\right.}\right] \hat{\mathcal{E}}_{\underline{\left.i_{\ell-1}\right\rangle}} \\
& -8 \hat{\Psi}^{\prime} \hat{\mathcal{E}}_{i_{\ell}}-2 \hat{\Phi} \hat{\mathcal{E}}_{\underline{i_{\ell}}}^{\prime}-8 \mathcal{H} \hat{\Phi} \hat{\mathcal{E}}_{i_{\ell}}, \\
& \mathcal{L}^{\#(2)}\left[\hat{\mathcal{B}}^{(2)}\right]_{\underline{i_{\ell}}}=\hat{\mathcal{B}}_{\underline{i_{\underline{\ell}}}}^{(2)}+4 \mathcal{H} \hat{\mathcal{B}}_{\underline{i_{\underline{\ell}}}}^{(2)}+\frac{(\ell-1)(\ell+3)}{(\ell+1)(2 \ell+3)} \partial^{J} \hat{\mathcal{B}}_{\underline{i_{\underline{i}}}}^{(2)}+\partial_{\left\langle I_{\ell}\right.} \hat{\mathcal{B}}_{\underline{\left.i_{\ell-1}\right\rangle}}^{(2)} \\
& +\frac{2}{(\ell+1)}\left(\operatorname{curl} \hat{\mathcal{E}}^{(2)}\right)_{\underline{i_{\ell}}} \text {, } \\
& \mathcal{L}^{\#(1)(1)}\left[\hat{\mathcal{B}}^{(1)}\right]_{\underline{i_{\ell}}}=\frac{2}{\ell+1} \epsilon_{j k\left\langle i_{\ell}\right.}\left[-2 \partial^{J}(\hat{\Phi}+\hat{\Psi}) \hat{\mathcal{E}}_{\underline{{ }_{i \ell-1}}}^{k}+2(\hat{\Phi}+\hat{\Psi}) \partial^{J} \hat{\mathcal{E}}^{k} \underline{\left.{ }_{i_{\ell-1}}\right\rangle}\right. \\
& \left.+8 \partial^{J} \hat{\Phi} \hat{\mathcal{E}}^{k}{ }_{\underline{\left.i_{\ell-1}\right\rangle}}\right] \text {. }
\end{aligned}
$$


4.3.1. Dependence on the choice of the tetrad We chose in section 3.2 .2 to align the tetrad $\mathbf{e}^{o}$ with the form $(\mathrm{d} \eta)$, which means that the corresponding observers follow worldlines always orthogonal to the constant time hypersurfaces. Alternatively, we could have chosen to align $\mathbf{e}_{o}$ with the vector $\left(\partial_{\eta}\right)$, which would then in turn correspond to observers of constant spatial coordinates [9]. These two choices are related by a boost parameterized by the shift vector $\omega^{I}$, and in the case of the Newtonian gauge, the parameter velocity which characterizes this boost reduces to $\hat{\Phi}^{I}$. This change in the tetrad used to decompose the momentum induces a transformation of the distribution function. Up to second order the two gauge invariant distribution functions (with obvious notation) are related by

$$
\begin{aligned}
& \hat{I}_{\left(\partial_{\eta}\right)}^{(1)}=\hat{I}_{(\mathrm{d} \eta)}^{(1)} \\
& \hat{I}_{\left(\partial_{\eta}\right)}^{(2)}=\hat{I}_{(\mathrm{d} \eta)}^{(2)}+\frac{\partial \bar{I}_{(\mathrm{d} \eta)}}{\partial p^{o}} p^{o} \hat{\Phi}_{I}^{(2)} n^{i} .
\end{aligned}
$$

It can be checked that the equation satisfied by $\hat{I}_{\left(\partial_{\eta}\right)}^{(2)}$ is the same as $\hat{I}_{(\mathrm{d} \eta)}^{(2)}$ [equation (4.21)] with $\partial_{I} \hat{\Phi}_{J}^{(2)} n^{i} n^{j}$ replaced by $-\hat{\Phi}_{J}^{\prime(2)} n^{j}$, and thus our result is consistent with [15, 48] where $\hat{I}_{\left(\partial_{\eta}\right)}^{(2)}$ is used.

What would be the best choice then? In our case (the same choice is performed in [9]), $e_{I}^{o}=0$. We can check that if there is no scalar perturbation, then the coordinates of the acceleration defined by $a_{\nu} \equiv\left(e^{o}\right)^{\mu} \nabla_{\mu} e_{\nu}^{o}$ read

$$
a_{I}=\left(e_{I}^{o}\right)^{\prime}+\mathcal{H} e_{I}^{o} .
$$

We thus conclude that the choice that we made corresponds to picking observers which accelerate the less, that is which accelerate only because of gradients in the gravitational potential, and thus this class of observers is the closest to freely falling observers.

\section{The gauge invariant Liouville equation for baryons}

\subsection{The background and first order Liouville operator}

We follow the same method as for radiation. The main difference lies in the fact that $n^{i}$ is not a unit vector anymore. Consequently we cannot drop terms like $n_{i} n^{i}=\beta^{2}$. Furthermore it is not interesting to split the dependence in the momentum into a dependence in its energy and in the direction of its spatial part, since the magnitude of the spatial momentum $(\lambda)$ cannot be identified with the energy $q^{o}$. At the background level, the evolution of $q^{o}, q^{i}$ and $\lambda$ are dictated by

$$
\left(\frac{\mathrm{d} q^{o}}{\mathrm{~d} \eta}\right)^{(0)}=-\mathcal{H} q^{o} \beta^{2}, \quad\left(\frac{\mathrm{d} \lambda}{\mathrm{d} \eta}\right)^{(0)}=-\mathcal{H} \lambda, \quad\left(\frac{\mathrm{d} q^{i}}{\mathrm{~d} \eta}\right)^{(0)}=-\mathcal{H} q^{i},
$$

and we thus obtain for the background Liouville operator

$$
\bar{L}^{\#}[\bar{g}]=\frac{\partial \bar{g}}{\partial \eta}-\mathcal{H} q^{i} \frac{\partial \bar{g}}{\partial q^{i}}=\frac{\partial \bar{g}}{\partial \eta}-\mathcal{H} \lambda \frac{\partial \bar{g}}{\partial \lambda} .
$$

In order to compute the Liouville operator at first order, we need the evolution of the particle spatial momentum magnitude $\lambda$ at first order, which from equation (4.3) is given in the Newtonian gauge by

$$
\left(\frac{\mathrm{d} \lambda}{\mathrm{d} \eta}\right)^{(1)}=\frac{1}{\beta}\left(\frac{\mathrm{d} q^{o}}{\mathrm{~d} \eta}\right)^{(1)}=\lambda\left[-\frac{1}{\beta^{2}} n^{i} \partial_{I} \Phi^{(1)}+\Psi^{(1)^{\prime}}\right] .
$$


and this leads to the following first order gauge invariant Liouville operator

$L^{\#(1)}\left[\bar{g}, \hat{g}^{(1)}\right]=\frac{\partial \hat{g}^{(1)}}{\partial \eta}+n^{j} \partial_{J} \hat{g}^{(1)}-\mathcal{H} q^{i} \frac{\partial \hat{g}^{(1)}}{\partial q^{i}}+\left[-\frac{1}{\beta^{2}} n^{j} \partial_{J} \hat{\Phi}^{(1)}+\hat{\Psi}^{(1)^{\prime}}\right] q^{k} \frac{\partial \bar{g}}{\partial q^{k}}$.

\subsection{The second order Liouville operator}

At second order, the evolution of $\lambda$ is also obtained from equation (4.3), and in the Newtonian gauge we obtain

$$
\begin{gathered}
\left(\frac{\mathrm{d} \lambda}{\mathrm{d} \eta}\right)^{(2)}=\frac{1}{\lambda}\left(\frac{\mathrm{d} q^{o}}{\mathrm{~d} \eta}\right)^{(2)}=\frac{\lambda}{\beta^{2}}\left\{-n^{i} \partial_{I} \Phi^{(2)}+\left[\Psi^{(2)^{\prime}} n_{i} n^{i}+\left(\partial_{I} B_{J}^{(2)}-H_{I J}^{(2)^{\prime}}\right) n^{i} n^{j}\right]\right. \\
\left.+2(\Phi-\Psi) n^{i} \partial_{I} \Phi+4 \beta^{2} \Psi \Psi^{\prime}\right\}
\end{gathered}
$$

We also need to use the first order evolution of $q^{i}$ which in the Newtonian gauge reads

$$
\left(\frac{\mathrm{d} q^{i}}{\mathrm{~d} \eta}\right)^{(1)}=q^{i} \Psi^{\prime}-q^{o} \partial^{I} \Phi-q^{o}\left(\beta^{2} \gamma^{i j}-n^{i} n^{j}\right) \partial_{J} \Psi .
$$

Finally we need the first order photon trajectory given in the Newtonian gauge by

$$
\left(\frac{\mathrm{d} x^{I}}{\mathrm{~d} \eta}\right)^{(1)}=\left(\frac{q^{i}}{q^{o}}\right)^{(1)}=n^{i}(\Phi+\Psi) .
$$

With this we obtain the second order gauge invariant Liouville operator for massive particles

$$
\begin{aligned}
L^{\#(2)}\left[\bar{g}, \hat{g}^{(2)}\right]= & \frac{\partial \hat{g}^{(2)}}{\partial \eta}+n^{j} \partial_{J} \hat{g}^{(2)}-\mathcal{H} q^{i} \frac{\partial \hat{g}^{(2)}}{\partial q^{i}} \\
& +\left[-\frac{1}{\beta^{2}} n^{j} \partial \hat{\Phi}^{(2)}+\hat{\Psi}^{(2)^{\prime}}+\frac{1}{\beta^{2}}\left(\partial_{I} \hat{\Phi}_{J}^{(2)}-\hat{H}_{I J}^{(2)^{\prime}}\right) n^{i} n^{j}\right] q^{k} \frac{\partial \bar{g}}{\partial q^{k}} \\
L^{\#(1)(1)}\left[\bar{g}, \hat{g}^{(1)}\right]= & 2 n^{i}(\hat{\Phi}+\hat{\Psi}) \partial_{I} \hat{g}^{(1)}-2\left[\left(\beta^{2} \delta^{i j}-n^{i} n^{j}\right) \partial \hat{\Psi}_{J}+\partial^{I} \hat{\Phi}\right] \frac{\partial \hat{g}^{(1)}}{\partial q^{i}} q^{o} \\
& +\left[\frac{2}{\beta^{2}}(\hat{\Phi}-\hat{\Psi}) n^{j} \partial_{J} \hat{\Phi}+4 \hat{\Psi} \hat{\Psi}^{\prime}\right] q^{i} \frac{\partial \bar{g}}{\partial q^{i}}+2 \frac{\partial \hat{g}^{(1)}}{\partial q^{i}} q^{i} \hat{\Psi}^{\prime}-2 \hat{\Phi} L^{\#(1)}\left[\bar{g}, \hat{g}^{(1)}\right]
\end{aligned}
$$

\subsection{The fluid limit}

If we choose a tetrad, not necessarily adapted to this bulk velocity, then the components of the stress-energy tensor are given in function of the energy density and pressure by

$$
\begin{aligned}
& T^{o o}=\rho+(\rho+P)\left[\left(u^{o}\right)^{2}-1\right]=\int \frac{\mathrm{d}^{3} q^{k}}{(2 \pi)^{3}} g\left(q^{k}\right) q^{o}, \\
& T^{o i}=\rho u^{o} u^{i}=\int \frac{\mathrm{d}^{3} q^{k}}{(2 \pi)^{3}} g\left(q^{k}\right) q^{i}, \\
& T^{i j}=\rho u^{i} u^{i}+P \delta^{i j}=\int \frac{\mathrm{d}^{3} q^{k}}{(2 \pi)^{3}} g\left(q^{k}\right) \frac{q^{i} q^{j}}{q^{o}} .
\end{aligned}
$$

In general for a distribution function $g\left(q^{b}\right)$, its associated stress-energy tensor conservation tensor $\nabla_{\mu} T^{\mu \nu}$ is obtained by [1]

$$
a \nabla_{\mu} T^{\mu b}=\int L^{\#}[g] q^{b} \frac{\mathrm{d}^{3} q^{i}}{(2 \pi)^{3}},
$$


where we recall that $a$ is the scale factor. We can then perform a perturbative expansion of this expression according to the expansion (2.7) and obtain for baryons the perturbation of the continuity equation

$$
\begin{aligned}
a \overline{\left(\nabla_{\mu} T^{\mu o}\right)}=\int & \frac{\mathrm{d}^{3} q^{k}}{(2 \pi)^{3}} q^{o} \bar{L}^{\#}[\bar{g}]=\left(\bar{T}^{o o}\right)^{\prime}+3 \mathcal{H} \bar{T}^{o o}+\mathcal{H} \bar{T}_{i}^{i}, \\
a\left(\nabla_{\mu} T^{\mu o}\right)^{(1)}= & \int \frac{\mathrm{d}^{3} q^{k}}{(2 \pi)^{3}} q^{o} L^{\#(1)}\left[\bar{g}, \hat{g}^{(1)}\right] \\
= & \left(\hat{T}^{o o(1)}\right)^{\prime}+3 \mathcal{H} \hat{T}^{o o(1)}+\mathcal{H} \hat{T}_{i}^{i(1)}+\partial_{I} \hat{T}^{o i(1)}-\hat{\Psi}^{\prime}\left(3 \bar{T}^{o o}+\bar{T}_{i}^{i}\right), \\
a\left(\nabla_{\mu} T^{\mu o}\right)^{(2)}= & \int \frac{\mathrm{d}^{3} q^{k}}{(2 \pi)^{3}} q^{o} L^{\#(2)}\left[\bar{g}, \hat{g}^{(1)}, \hat{g}^{(2)}\right] \\
= & \left(\hat{T}^{o o(2)}\right)^{\prime}+3 \mathcal{H} \hat{T}^{o o(2)}+\mathcal{H} \hat{T}_{i}^{i(2)}+\partial_{I} \hat{T}^{o i(2)}-\hat{\Psi}^{\prime}(2)\left(3 \bar{T}^{o o}+\bar{T}_{i}^{i}\right) \\
& +2(\hat{\Psi}+\hat{\Phi}) \partial_{I} \hat{T}^{o i(1)}-2 \hat{\Psi}^{\prime}\left(3 \hat{T}^{o o(1)}+\hat{T}_{, i}^{i(1)}\right)+4 \hat{T}^{o i(1)} \partial_{I}(\hat{\Phi}-\hat{\Psi}) \\
& -4\left(3 \bar{T}^{o o}+\bar{T}_{\mathrm{b}, i}^{i}\right) \hat{\Psi}^{\prime} \hat{\Psi} \\
& -2 \hat{\Phi}\left[\left(\hat{T}^{o o(1)}\right)^{\prime}+3 \mathcal{H} \hat{T}^{o o(1)}+\mathcal{H} \hat{T}_{i}^{i(1)}+\partial_{I} \hat{T}^{o i(1)}-\hat{\Psi}^{\prime}\left(3 \bar{T}^{o o}+\bar{T}_{i}^{i}\right)\right],
\end{aligned}
$$

and the perturbation of the Euler equation

$$
\begin{aligned}
a\left(\nabla_{\mu} T^{\mu i}\right)^{(0)}= & 0 \\
a\left(\nabla_{\mu} T^{\mu i}\right)^{(1)}= & \int \frac{\mathrm{d}^{3} q^{k}}{(2 \pi)^{3}} q^{i} L^{\#(1)}\left[\bar{g}, \hat{g}^{(1)}\right] \\
= & \left(\hat{T}^{o i(1)}\right)^{\prime}+4 \mathcal{H} \hat{T}^{o i(1)}+\partial_{J} \hat{T}^{j i(1)}+\partial_{J} \hat{\Phi}\left(\bar{T}^{o o} \delta^{i j}+\bar{T}^{i j}\right), \\
a\left(\nabla_{\mu} T^{\mu i}\right)^{(2)}= & \int \frac{\mathrm{d}^{3} q^{k}}{(2 \pi)^{3}} q^{i} L^{\#(2)}\left[\bar{g}, \hat{g}^{(1)}, \hat{g}^{(2)}\right] \\
= & \left(\hat{T}^{o i(2)}\right)^{\prime}+4 \mathcal{H} \hat{T}^{o i(2)}+\partial_{J} \hat{T}^{j i(2)}+\partial_{J} \hat{\Phi}^{(2)}\left(\bar{T}^{o o} \delta^{i j}+\bar{T}^{i j}\right) \\
& -2 \hat{\Phi}\left[\left(\hat{T}^{o i(1)}\right)^{\prime}+4 \mathcal{H} \hat{T}^{o i(1)}\right]+(2 \hat{\Psi}-4 \hat{\Phi}) \partial_{J} \hat{\Phi}^{(1)}\left(\bar{T}^{o o} \delta^{i j}+\bar{T}^{i j}\right) \\
& +2 \partial_{J} \hat{\Phi}\left(\hat{T}^{o o(1)} \delta^{i j}+\hat{T}^{i j(1)}\right)-8 \hat{\Psi}^{\prime} \hat{T}^{o i(1)}+2 \hat{\Psi} \partial_{J} \hat{T}^{i j(1)} \\
& +2\left(\partial^{I} \hat{\Psi} \hat{T}_{k}^{k(1)}-3 \partial_{J} \hat{\Psi} \hat{T}^{i j(1)}\right) .
\end{aligned}
$$

In order to further specify the continuity and Euler equations, we need to express the perturbations of the stress-energy tensor components in function of the perturbations of the fluid quantities. Due to the strong coupling between electrons and protons through Coulomb interactions when matter is ionized, the baryons cannot develop significant anisotropic stress before recombination and thus the corresponding stress-energy tensor is of the form

$$
T^{\mu \nu}=(P+\rho) u^{\mu} u^{\nu}+P g^{\mu \nu} .
$$


Indeed, the strong interactions between electrons and protons ensure that they follow a Fermi-Dirac distribution of energies in their rest frame (that is where they have no bulk velocity). We also note that since $\bar{u}^{i}=0$, then the normalization condition of $u_{a} u^{a}=-1$ implies

$$
u^{o(1)}=0, \quad u^{o(2)}=u^{i(1)} u_{i(1)} .
$$

Furthermore we can identify the velocity $v^{i}$ and the spatial momentum $u^{i}$ up to second order in perturbation since

$$
u^{i}=\frac{1}{\sqrt{1-v_{j} v^{j}}} v^{i} .
$$

We then deduce the following perturbative expansion of the stress-energy tensor in function of the fluid quantities

$$
\begin{aligned}
\bar{T}^{o o} & =\bar{\rho}, \\
T^{o o(1)} & =\rho^{(1)} \\
T^{o o(2)} & =\rho^{(2)}+2(\bar{\rho}+\bar{P}) u^{i(1)} u_{i}^{(1)}, \\
\bar{T}^{o i} & =0, \\
T^{o i(1)} & =(\bar{\rho}+\bar{P}) u^{i(1)}, \\
T^{o i(2)} & =(\bar{\rho}+\bar{P})\left(u^{i(2)}\right)+2\left(\rho^{(1)}+P^{(1)}\right) u^{i(1)}, \\
\bar{T}^{i j} & =\bar{P} \delta^{i j} \\
T^{i j(1)} & =P^{(1)} \delta^{i j}, \\
T^{i j(2)} & =P^{(2)} \delta^{i j}+2(\bar{\rho}+\bar{P}) u^{i(1)} u^{j(1)} .
\end{aligned}
$$

By using these expressions, we obtain the following gauge invariant conservation and Euler equations from equations 5.14 (5.19)

$$
\begin{aligned}
a \overline{\left(\nabla_{\mu} T^{\mu o}\right)}=\bar{\rho}^{\prime}+ & 3 \mathcal{H}(\bar{\rho}+\bar{P}) \\
a\left(\nabla_{\mu} T^{\mu o}\right)^{(1)}= & \hat{\rho}^{(1)^{\prime}}+3 \mathcal{H}\left(\hat{\rho}^{(1)}+\hat{P}^{(1)}\right) \\
& +(\bar{\rho}+\bar{P}) \partial_{I} \hat{u}^{i(1)}-3(\bar{\rho}+\bar{P}) \hat{\Psi}^{\prime} \\
a\left(\nabla_{\mu} T^{\mu o}\right)^{(2)}= & \hat{\rho}^{(2)^{\prime}}+3 \mathcal{H}\left(\hat{\rho}^{(2)}+\hat{P}^{(2)}\right)+6 \mathcal{H}(\bar{\rho}+\bar{P}) \hat{u}^{i(1)} \hat{u}_{i}^{(1)} \\
& +(\bar{\rho}+\bar{P}) \partial_{I} \hat{u}^{i(2)}-3(\bar{\rho}+\bar{P}) \hat{\Psi}^{(2)^{\prime}}+2\left(\bar{\rho}^{\prime}+\bar{P}^{\prime}\right) \hat{u}^{i(1)} \hat{u}_{i}^{(1)} \\
& +2 \mathcal{H}(\bar{\rho}+\bar{P}) \hat{u}^{i(1)} \hat{u}_{i}^{(1)}+2 \partial_{I}\left[\left(\hat{\rho}^{(1)}+\hat{P}^{(1)}\right) \hat{u}^{i(1)}\right]-6 \hat{\Psi}^{\prime}\left(\hat{\rho}^{(1)}+\hat{P}^{(1)}\right) \\
& +2(\hat{\Phi}+\hat{\Psi})(\bar{\rho}+\bar{P}) \partial_{I} \hat{u}^{i(1)}+4(\bar{\rho}+\bar{P}) \hat{u}^{i(1)} \partial_{I}\left(\hat{\Phi}^{(1)}-\hat{\Psi}^{\prime}\right) \\
& -12 \hat{\Psi} \hat{\Psi}^{\prime}(\bar{\rho}+\bar{P})+4(\bar{\rho}+\bar{P}) \hat{u}^{i(1)} \hat{u}_{i}^{(1)^{\prime}}-2 \hat{\Phi} a\left(\nabla_{\mu} T^{\mu o}\right)^{(1)}
\end{aligned}
$$




$$
\begin{aligned}
a\left(\nabla_{\mu} T^{\mu i}\right)^{(1)}= & {\left[(\bar{\rho}+\bar{P}) \hat{u}^{i(1)}\right]^{\prime}+4 \mathcal{H}\left[(\bar{\rho}+\bar{P}) \hat{u}^{i(1)}\right] } \\
& +\partial^{I} \hat{P}^{(1)}+\partial^{I} \hat{\Phi}(\bar{\rho}+\bar{P}) \\
a\left(\nabla_{\mu} T^{\mu i}\right)^{(2)}= & {\left[(\bar{\rho}+\bar{P}) \hat{u}^{i(2)}\right]^{\prime}+4 \mathcal{H}\left[(\bar{\rho}+\bar{P}) \hat{u}^{i(2)}\right] } \\
& +\partial^{I} \hat{P}^{(2)}+(\bar{\rho}+\bar{P}) \partial^{I} \hat{\Phi}^{(2)}+2(\bar{\rho}+\bar{P}) \partial_{J}\left(\hat{u}^{i(1)} \hat{u}^{j(1)}\right) \\
& +2\left[\left(\hat{\rho}^{(1)}+\hat{P}^{(1)}\right) \hat{u}^{i(1)}\right]^{\prime}+8 \mathcal{H}\left[\left(\hat{\rho}^{(1)}+\hat{P}^{(1)}\right) \hat{u}^{i(1)}\right] \\
& -2(\hat{\Phi}+\hat{\Psi})\left\{\left[(\bar{\rho}+\bar{P}) \hat{u}^{i(1)}\right]^{\prime}+4 \mathcal{H}\left[(\bar{\rho}+\bar{P}) \hat{u}^{i(1)}\right]\right\} \\
& -4(\bar{\rho}+\bar{P}) \hat{\Phi} \partial^{I} \hat{\Phi}+2 \partial^{I} \hat{\Phi}\left(\hat{\rho}^{(1)}+\hat{P}^{(1)}\right)-8 \hat{\Psi}^{\prime}(\bar{\rho}+\bar{P}) \hat{u}^{i(1)} \\
& +2 \hat{\Psi} a\left(\nabla \mu T^{\mu i}\right)^{(1)} .
\end{aligned}
$$

\subsection{Comparison with the coordinates approach}

In the literature, the fluid equation are usually derived starting directly from the stressenergy tensor and in components associated with coordinates. It is straightforward to compare with our results since

$$
\nabla_{\mu} T^{\mu A}=\nabla_{\mu} T^{\mu i} e_{i}^{A} .
$$

At first order we have

$$
a\left(\nabla_{\mu} T^{\mu A}\right)^{(1)}=\left(\nabla_{\mu} T^{\mu a}\right)^{(1)},
$$

and at second order

$$
\begin{aligned}
& a\left(\nabla_{\mu} T^{\mu O}\right)^{(2)}=\left[\left(\nabla_{\mu} T^{\mu o}\right)^{(2)}+2 \hat{\Phi}^{(1)}\left(\nabla_{\mu} T^{\mu o}\right)^{(1)}\right], \\
& a\left(\nabla_{\mu} T^{\mu I}\right)^{(2)}=\left[\left(\nabla_{\mu} T^{\mu i}\right)^{(2)}-2 \hat{\Psi}^{(1)}\left(\nabla_{\mu} T^{\mu i}\right)^{(1)}\right] .
\end{aligned}
$$

The presentation that we have chosen in the previous section makes the use of these relations simple. However, in order to succeed this comparison it should be noticed that 45

$$
\begin{aligned}
& v^{i(1)}=V^{I(1)}, \\
& v^{i(2)}=V^{I(2)}-2 \Psi V^{I(1)},
\end{aligned}
$$

since the results in the literature for the fluid approximation are so far expressed in function of $V^{I}$. 


\section{Collision term}

\subsection{General expression}

The general expression of the collision term is given by [70, 79, 80,

$$
\begin{aligned}
& C_{\mathbf{u} \kappa \omega}(\mathbf{p})=S_{\mathbf{u}_{\kappa}}{ }_{\kappa} S_{\mathbf{u}_{\omega}{ }^{\beta}}(2 \pi)^{4} \int \mathrm{D} \mathbf{q} \int \mathrm{D}^{\prime} \int \mathrm{D} \mathbf{q}^{\prime} \delta_{D}^{4}\left(\mathbf{p}^{\prime}+\mathbf{q}-\mathbf{p}-\mathbf{q}^{\prime}\right) \\
& \left\{g_{\mathrm{e}}(\mathbf{q}) f_{\mu \nu}\left(\mathbf{p}^{\prime}\right) \mathcal{M}_{\alpha \beta}^{\mu \nu}\left(\mathbf{p}^{\prime}, \mathbf{q} ; \mathbf{p}, \mathbf{q}^{\prime}\right)\right. \\
& -g_{\mathrm{e}}\left(\mathbf{q}^{\prime}\right)\left[\eta^{\sigma \tau} U_{\alpha \beta}(\mathbf{p}) f_{\mu \nu}(\mathbf{p})\right] \mathcal{M}_{\sigma \tau}^{\mu \nu}\left(\mathbf{p}, \mathbf{q}^{\prime} ; \mathbf{p}^{\prime}, \mathbf{q}\right) \\
& \left.+\left[g_{\mathrm{e}}(\mathbf{q})-g_{\mathrm{e}}\left(\mathbf{q}^{\prime}\right)\right] f_{\mu \nu}\left(\mathbf{p}^{\prime}\right) \mathcal{M}_{\sigma \tau}^{\mu \nu}\left(\mathbf{p}^{\prime}, \mathbf{q} ; \mathbf{p}, \mathbf{q}^{\prime}\right) \mathcal{N}_{\mathbf{q}}{ }^{\sigma \tau}{ }_{\alpha \beta}(\mathbf{p})\right\},
\end{aligned}
$$

where we used the notation D $p \equiv \delta_{D}^{1}\left(\mathbf{p} \cdot \mathbf{p}-m^{2}\right) \frac{\mathrm{d}^{4} p^{a}}{(2 \pi)^{3}}$. This expression accounts for the incoming transitions in the second line and the outgoing transitions in the third line. We have neglected the Pauli blocking terms $\left(1-g_{\mathrm{e}} / 2\right)$ since for the physics of the $\mathrm{CMB}$, the electrons are much more diluted than the photons. However we include the stimulated emission in the fourth line and discuss later its relevance, and this requires the use of the stimulated emission matrix

$\mathcal{N}_{\mathbf{q}}{ }^{\sigma \tau}{ }_{\alpha \beta}(\mathbf{p}, \mathbf{q}) \equiv$

$\frac{1}{2}\left[f_{\alpha \beta}(\mathbf{p}) S_{\mathbf{q}}{ }^{\sigma \tau}+\left(S_{\mathbf{q}_{\alpha}}{ }^{\sigma} S_{\mathbf{q} \beta}{ }^{\tau}-S_{\mathbf{q}}{ }^{\sigma \tau} S_{\mathbf{q}_{\alpha \beta}}\right) I(\mathbf{p})+f^{\star \sigma \tau}(\mathbf{p}) S_{\mathbf{q}_{\alpha \beta}}\right]$.

In this definition we recall that the projectors $S_{\mathbf{q}}{ }^{\alpha \beta}$ are taken with respect to an observer having a velocity $\mathbf{q} / m$, which means that $S_{\mathbf{q}}{ }^{\alpha \beta} q_{\beta}=0$, and $f^{\star \sigma \tau}$ is the complex conjugate of $f^{\sigma \tau}$. The stimulated emission in the case of polarized light is discussed in 64, 80, 81. These treatments give the stimulated emission matrix in terms of the Stokes parameters. However, we do not want to refer to them since they imply and choice of the angle in the polarization plane basis, but it can be checked that the expression given here for the stimulated emission tensor is equivalent to the expression of these references when expressed in terms of the Stokes parameters. These parameters are an intermediary step between the polarization tensor and the multipoles which is not compulsory, and we thus intentionally bypass them in our derivations. In the end, this method will prove more powerful because it is computationally more straightforward.

The Dirac function in equation (6.1) ensures momentum conservation and $\mathcal{M}^{\mu \nu}{ }_{\sigma \tau}\left(\mathbf{p}, \mathbf{q} ; \mathbf{p}^{\prime}, \mathbf{q}^{\prime}\right)$ is the transition tensor for the process

$$
\gamma(\mathbf{p})+e^{-}(\mathbf{q}) \longleftrightarrow \gamma\left(\mathbf{p}^{\prime}\right)+e^{-}\left(\mathbf{q}^{\prime}\right) .
$$

Its expression is given by

$$
\begin{aligned}
& \mathcal{M}^{\mu \nu}{ }_{\alpha \beta}\left(\mathbf{p}, \mathbf{q} ; \mathbf{p}^{\prime}, \mathbf{q}^{\prime}\right)= \\
& \frac{3}{2} \pi \sigma_{\mathrm{T}} m_{\mathrm{e}}^{2}\left\{{Q_{\mathbf{q}}{ }_{\alpha \beta}{ }^{\mu \nu}}^{\prime}+\frac{1}{4}\left[\frac{\mathbf{p}^{\prime} \cdot \mathbf{q}}{\mathbf{p} \cdot \mathbf{q}}+\frac{\mathbf{p . q}}{\mathbf{p}^{\prime} \cdot \mathbf{q}}-2\right]\left(S_{\mathbf{q}}{ }^{\mu \nu} S_{\mathbf{q}_{\alpha \beta}}^{\prime}+{Q_{\mathbf{q}}{ }_{\alpha \beta}}^{\mu \nu}-Q_{\mathbf{q}_{\alpha \beta}}{ }^{\nu \mu}\right)\right\}
\end{aligned}
$$

where

$$
Q_{\mathbf{q}_{\alpha \beta}}^{\mu \nu} \equiv S_{\mathbf{q}}{ }_{\gamma}^{\mu} S_{\mathbf{q} \delta}{ }^{\nu} S_{\mathbf{q} \alpha}^{\prime}{ }_{\alpha}^{\gamma} S_{\mathbf{q}_{\beta}}^{\prime \delta} \equiv S_{\mathbf{q}}{ }_{\gamma}^{\mu}(\mathbf{p}) S_{\mathbf{q} \delta}{ }^{\nu}(\mathbf{p}) S_{\mathbf{q}}{ }_{\alpha}^{\gamma}\left(\mathbf{p}^{\prime}\right) S_{\mathbf{q}}{ }_{\beta}^{\delta}\left(\mathbf{p}^{\prime}\right)
$$

The detail of its derivation can be found in [79. Intuitively, it consists in transforming the incoming polarization tensor to the rest frame of the incoming electron, and 
applying the Klein-Nishina formula for the amplitude of the scattering process. Thanks to the reversibility of the scattering process, the transition tensor satisfies

$$
\mathcal{M}_{\alpha \beta}^{\mu \nu}\left(\mathbf{p}^{\prime}, \mathbf{q}^{\prime} ; \mathbf{p}, \mathbf{q}\right)=S_{\mathbf{q}^{\prime}}{ }_{\omega}^{\mu} S_{\mathbf{q}^{\prime}}{ }_{\tau}^{\nu} \mathcal{M}^{\omega \tau}{ }_{\sigma \lambda}\left(\mathbf{p}, \mathbf{q} ; \mathbf{p}^{\prime}, \mathbf{q}^{\prime}\right) S_{\mathbf{q}^{\prime}}{ }_{\alpha}{ }_{\alpha} S_{\mathbf{q}^{\prime}}{ }_{\beta}{ }_{\beta},
$$

as it can be checked directly on its expression (6.4). It is then possible to recast the collision term as

$$
\begin{aligned}
& C_{\mathbf{u} \kappa \omega}(\mathbf{p})=S_{\mathbf{u}}{ }_{\kappa}^{\alpha} S_{\mathbf{u} \omega}^{\beta} \int \mathrm{Dq} \int \mathrm{D}^{\prime} \int \mathrm{Dq}^{\prime}(2 \pi)^{4} \delta_{D}^{4}\left(\mathbf{p}^{\prime}+\mathbf{q}-\mathbf{p}-\mathbf{q}^{\prime}\right) \\
& \times g_{\mathrm{e}}(\mathbf{q}) f_{\mu \nu}\left(\mathbf{p}^{\prime}\right) \mathcal{M}^{\mu \nu}{ }_{\sigma \tau}\left(\mathbf{p}^{\prime}, \mathbf{q} ; \mathbf{p}, \mathbf{q}^{\prime}\right)\left\{\delta_{\alpha}^{\sigma} \delta_{\beta}^{\tau}+\mathcal{N}_{\mathbf{q}}{ }^{\sigma \tau}{ }_{\alpha \beta}(\mathbf{p})\right\} \\
& -S_{\mathbf{u} \kappa}^{\alpha} S_{\mathbf{u} \omega}^{\beta} \int \operatorname{DqD}^{\prime} \operatorname{Dq}^{\prime}(2 \pi)^{4} \delta_{D}^{4}\left(\mathbf{p}+\mathbf{q}-\mathbf{p}^{\prime}-\mathbf{q}^{\prime}\right) \\
& \times g_{\mathrm{e}}(\mathbf{q})\left\{\eta^{\sigma \tau} U_{\alpha \beta}(\mathbf{p}) f_{\mu \nu}(\mathbf{p})+\mathcal{N}_{\mathbf{q}_{\mu \nu \alpha \beta}}(\mathbf{p}) f^{\sigma \tau}\left(\mathbf{p}^{\prime}\right)\right\} \mathcal{M}_{\sigma \tau}^{\mu \nu}\left(\mathbf{p}, \mathbf{q} ; \mathbf{p}^{\prime}, \mathbf{q}^{\prime}\right) .
\end{aligned}
$$

The remaining two screen projectors at the very beginning of equations (6.16.7) ensure that the result is read in the required frame (usually the cosmological frame). The form of the expressions (6.16.7) is manifestly covariant. First, the measures Dp and Dq, the Dirac function and the electron distribution function $g_{\mathrm{e}}(\mathbf{p})$ are Lorentz invariant. Second, given the transformation rule (1.46) for the polarization tensor, the screen projectors in the transition tensor and the stimulated emission tensor ensure that $f_{\mu \nu}\left(\mathbf{p}^{\prime}\right) \mathcal{M}_{\alpha \beta}^{\mu \nu}\left(\mathbf{p}^{\prime}, \mathbf{q} ; \mathbf{p}, \mathbf{q}^{\prime}\right)$ and $f_{\mu \nu}\left(\mathbf{p}^{\prime}\right) \mathcal{M}^{\mu \nu}{ }_{\sigma \tau}\left(\mathbf{p}^{\prime}, \mathbf{q} ; \mathbf{p}, \mathbf{q}^{\prime}\right) f_{\mathbf{q}}^{\star \sigma \tau}(\mathbf{p})$ are independent from the frame used to evaluate $f_{\mu \nu}$. Finally we note that the screen projectors $S_{\mathbf{u} \kappa}^{\alpha} S_{\mathbf{u}}{ }_{\omega}^{\beta}$ are defined with respect to the observer having a velocity $\mathbf{u}$, which is the velocity with respect to which the collision tensor in equations (6.116.7) is defined (in our case $\left.u_{\mu}=(\mathrm{d} \eta)_{\mu}\right)$. Hence they ensure that the expression of the collision tensor is independent from the observer (that is the frame) used to define the unit polarization tensor $U_{\alpha \beta}$ appearing in the third line of equation (6.1) and the fourth line of equation (6.7), and also independent from the observer used to define the polarization tensor $f_{\alpha \beta}$ appearing in the stimulated emission tensor. Additionally these screen projectors makes it straightforward to check that the collision tensor transforms as in the rule (1.46).

Given this discussion, it is in principle possible to express this collision term in the fundamental tetrad basis, in order to specify further the collision tensor. However, all the screen projectors appearing in the transition matrix and in the stimulated emission tensor are taken with respect to the observer with velocity $\mathbf{q}$ which varies throughout the integration $\int$ Dq. In order to simplify the computations, it would thus be helpful to use an intermediate tetrad basis adapted to each momentum $\mathbf{q}$ in this integration. Additionally, instead of expressing the collision tensor in the fundamental frame or tetrad, one would prefer to compute it, that is to obtain it explicitly in function of the radiation multipoles, in the electrons rest frame. In a second step, we would then transform the collision term in the fundamental frame using its transformation rule which is given by equation (1.46), and the transformation rules of the radiation multipoles and momentum components under this change of frame that we gave in section 1.7. We thus need for this final step to determine up to which order in the electrons velocity we need to compute this change of frame to be consistent with the perturbative expansion of the Boltzmann equation. 


\subsection{A double perturbative expansion}

When performing a perturbative expansion, we need to expand both in the metric perturbations and in the velocity perturbations. However these perturbations are of different type and also have a different magnitude. The magnitude of the metric perturbations are typically of order $10^{-5}$ for the processes involved in the CMB, and thus the bulk velocities of the radiation and matter distribution are also of the same amplitude. However the temperature of radiation $\left(T_{\mathrm{r}}\right)$ and the temperature of electrons $\left(T_{\mathrm{e}}\right)$, which are nearly identical around recombination are approximately of order $T_{\mathrm{r}} / m_{\mathrm{e}} \simeq T_{\mathrm{e}} / m_{\mathrm{e}} \simeq 10^{-6}$ and thus the thermal velocity of electrons is of order $10^{-3}$. Consequently, when going up to second order in metric perturbations, we would need in principle to keep perturbations up to fourth order in velocities as they can in the end lead to terms of order $\left(T_{\mathrm{r}} / m_{\mathrm{e}}\right)^{2}, T_{\mathrm{r}} T_{\mathrm{e}} / m_{\mathrm{e}}^{2}$ and $\left(T_{\mathrm{e}} / m_{\mathrm{e}}\right)^{2}$, which can be comparable to the second order perturbations of the metric if the numeric coefficients in front of these terms are quite large. It can be shown 82, 83, 84 that in fact, due to Comptonization processes, these terms will be of order $\left(\bar{T} / m_{\mathrm{e}}\right)^{2} \times\left(T_{\mathrm{r}}-T_{\mathrm{e}}\right) / \bar{T}$ and will be thus completely negligible in our context. However we would certainly need to retain terms up to third order in the baryons velocity, that we will call accordingly to this discussion of order $3 / 2$ in the metric perturbation (though there is no such half integer perturbation for the metric).

We will now apply this method to perform a perturbative expansion of the collision term

- in section 6.3 for electrons having no thermal dispersion and choosing for this computation the rest frame of electrons which is also the rest frame of baryons,

- in section 6.4 for electrons having a thermal dispersion, choosing the same frame for the computation,

- in section 6.5 for the general case, accounting for the bulk velocity of electrons by transforming the result obtained in the baryons rest frame toward the fundamental frame.

\subsection{Distribution of cold electrons with no bulk velocity}

It will prove convenient to calculate the collision term in the Boltzmann equation starting from a very simple case. We will first assume that the free electrons have only a bulk velocity and no thermal dispersion in their velocity distribution function $g_{\mathrm{e}}\left(q^{i}\right)$. Furthermore, we will choose to align the first vector of the tetrad $\mathbf{e}_{o}$ (an adapted tetrad) with this bulk velocity that is to work in the baryons rest frame in order to specify the collision tensor given in equation (6.7). In that case the electron distribution function is explicitly given by $g_{\mathrm{e}}\left(q^{i}\right)=(2 \pi)^{3} \delta_{D}^{3}\left(q^{i}\right) n_{\mathrm{e}}$ which makes the integration on electrons momenta trivial. In the baryons rest frame, the velocity of the incoming electron is aligned with the tetrad $\mathbf{e}_{o}$, and we obtain from the momentum conservation in the collision

$$
\frac{1}{q^{o} q^{\prime o}}=\frac{1}{m_{\mathrm{e}}^{2}}\left[1-\frac{\left(p^{i}-p^{\prime i}\right)\left(p_{i}-p_{i}^{\prime}\right)}{2 m_{\mathrm{e}}^{2}}\right] .
$$

The second term in the brackets of this expression is of order $\left(T_{\mathrm{r}} / m_{\mathrm{e}}\right)^{2}$, that is comparable to second order perturbations in the metric, and according to the discussion in section 6.2 they can be ignored. We then need to expand the Dirac 
function. We first integrate on the spatial components of the outgoing electron momentum since the integrand does not depend on it in the form (6.7) of the collision tensor. The remaining one-dimensional Dirac function is handled by following the standard steps that can be found in 2, and we obtain

$\delta_{D}^{1}\left(p^{\prime o}+q^{o}-p^{o}-q^{o o}\right)=\delta_{D}^{1}\left(p^{o}-p^{o}\right)-\frac{\left(p^{i}-p^{i}\right)\left(p_{i}-p_{i}^{\prime}\right)}{2 m_{\mathrm{e}}} \frac{\partial}{\partial p^{\prime o}} \delta_{D}^{1}\left(p^{\prime o}-p^{o}\right)$,
$\delta_{D}^{1}\left(p^{\prime o}+q^{\prime o}-p^{o}-q^{o}\right)=\delta_{D}^{1}\left(p^{o}-p^{o o}\right)+\frac{\left(p^{i}-p^{\prime i}\right)\left(p_{i}-p_{i}^{\prime}\right)}{2 m_{\mathrm{e}}} \frac{\partial}{\partial p^{\prime o}} \delta_{D}^{1}\left(p^{\prime o}-p^{o}\right)$.

Additionally, using the fact the tetrad corresponds to the rest frame of all electrons,

$$
\frac{1}{p^{o}}=\frac{1}{p^{\prime o}}+\frac{1}{m_{\mathrm{e}}}\left(1-n^{i} n_{i}^{\prime}\right),
$$

which is more conveniently expressed as

$$
\frac{p^{\prime o}}{p^{o}}=1+\frac{p^{\prime o}}{m_{\mathrm{e}}}\left(1-n^{i} n_{i}^{\prime}\right) .
$$

As a consequence, the term in square brackets in the definition (6.4) of the transition matrix is of order $\left(p^{o} / m_{\mathrm{e}}\right)^{2} \sim\left(T_{\mathrm{r}} / m_{\mathrm{e}}\right)^{2}$ and is comparable to second order perturbations of the metric. This would not have been the case if the tetrad was not adapted to the incoming electron velocity and in that case the term in brackets in equation (6.4) would be of order $T_{\mathrm{r}} / m_{\mathrm{e}}$. This property is important since, given the discussion in section 6.2, they can be ignored. Finally using

$$
n_{\mathrm{e}}=\int \frac{\mathrm{d}^{3} q^{i}}{(2 \pi)^{3}} g_{\mathrm{e}}\left(q^{h}\right),
$$

the collision term is expressed as

$$
C_{a b}\left(p^{h}\right)=n_{\mathrm{e}} \sigma_{\mathrm{T}}\left[C_{a b, \mathrm{~T}}\left(p^{h}\right)+C_{a b, \mathrm{R}}\left(p^{h}\right)\right],
$$

where

$$
\begin{aligned}
& C_{a b, \mathrm{~T}}\left(p^{h}\right)=p^{o}\left[\frac{3}{2} \int \frac{\mathrm{d}^{2} \Omega^{\prime}}{4 \pi} S_{a}^{c} S_{b}^{d} f_{c d}\left(p^{\prime h}\right)-f_{a b}\left(p^{h}\right)\right] \\
& C_{a b, \mathrm{R}}\left(p^{h}\right)=-\frac{3}{2} \int \mathrm{d} p^{\prime o} p^{\prime o} \int \frac{\mathrm{d}^{2} \Omega^{\prime}}{4 \pi}\left[\frac{\left(p^{o}\right)^{2}+\left(p^{\prime o}\right)^{2}-2 p^{\prime o} p^{o} n^{i} n_{i}^{\prime}}{2 m_{\mathrm{e}}}\right] \frac{\partial \delta_{D}^{1}\left(p^{\prime o}-p^{o}\right)}{\partial p^{\prime o}}(6.16) \\
& \times\left\{S^{c d} f_{c d}\left(p^{\prime h}\right)\left[f_{a b}\left(p^{h}\right)-I\left(p^{h}\right) \frac{S_{a b}}{2}\right]+\left[1+I\left(p^{h}\right)\right] S_{a}^{c} S_{b}^{d} f_{c d}\left(p^{\prime h}\right)\right\}+2 \frac{\left(p^{o}\right)^{2}}{m_{\mathrm{e}}} f_{a b}\left(p^{h}\right) .
\end{aligned}
$$

We recall that in these expressions, the screen projector $S^{c d}$ is defined with respect to the photon with momentum $\mathbf{p}$, and thus $S^{c d} f_{c d}\left(p^{\prime h}\right) \neq I\left(p^{\prime h}\right)$. Note also that $S^{c d} S_{c d}^{\prime}=1+\left(\mathbf{n} \cdot \mathbf{n}^{\prime}\right)^{2}[79$, and this helps recovering from the trace of the Thomson term (6.15) the standard form of the Thomson collision term for unpolarized radiation. Using the integrals (1.52) we find explicitly the Thomson and the recoil terms which are given by

$$
\begin{aligned}
\frac{C_{a b, \mathrm{~T}}\left(p^{h}\right)}{p^{o}}= & \frac{1}{2} S_{a b}\left[-I\left(p^{o}, n^{i}\right)+I_{\emptyset}\left(p^{o}\right)+\frac{1}{10} I_{c d}\left(p^{o}\right) n^{c} n^{d}-\frac{3}{5} E_{c d}\left(p^{o}\right) n^{c} n^{d}\right] \\
& +\left[-P_{a b}\left(p^{o}, n^{i}\right)-\frac{1}{10} I_{a b}\left(p^{o}\right)+\frac{3}{5} E_{a b}\left(p^{o}\right)\right]^{\mathrm{TT}} \\
& +\frac{1}{2} \mathrm{i} \epsilon_{a b c} n^{c}\left[-V\left(p^{o}, n^{i}\right)+\frac{1}{2} V_{d}\left(p^{o}\right) n^{d}\right],
\end{aligned}
$$




$$
\begin{gathered}
\frac{C_{a b, \mathrm{R}}\left(p^{h}\right)}{p^{o}}=\frac{p^{o}}{m_{\mathrm{e}}}\left\{\frac { 1 } { 2 } S _ { a b } \left\{2 I\left(p^{o}, n^{i}\right)+\left[1+I\left(p^{o}, n^{i}\right)\right]\left(2+p^{o} \frac{\partial}{\partial p^{o}}\right)\right.\right. \\
{\left[I_{\emptyset}\left(p^{o}\right)-\frac{2}{5} I_{c}\left(p^{o}\right) n^{c}+\frac{1}{10} I_{c d}\left(p^{o}\right) n^{c} n^{d}-\frac{3}{70} I_{c d h}\left(p^{o}\right) n^{c} n^{d} n^{h}\right.} \\
\left.\left.-\frac{3}{5} E_{c d}\left(p^{o}\right) n^{c} n^{d}+\frac{1}{7} E_{c d h}\left(p^{o}\right) n^{c} n^{d} n^{h}\right]\right\} \\
+\left\{P_{a b}\left(p^{o}, n^{i}\right)\left[2+I_{\emptyset}^{\prime}\left(p^{o}\right)+2 I_{\emptyset}\left(p^{o}\right)\right]+\left[1+I_{\emptyset}\left(p^{o}\right)\right]\left(2+p^{o} \frac{\partial}{\partial p^{o}}\right)\right. \\
{\left[+\frac{3}{5} E_{a b}\left(p^{o}\right)-\frac{1}{7} E_{a b c}\left(p^{o}\right) n^{c}-\frac{2}{5} B_{c a}\left(p^{o}\right) \epsilon_{b}^{c d} n_{d}\right.} \\
+\frac{1}{10} I_{a b}\left(p_{a b c}^{o} n^{c}\left\{V\left(p^{o}, n^{i}\right)\left[2+I_{\emptyset}^{\prime}\left(p^{o}\right)+2 I_{\emptyset}\left(p^{o}\right)\right]+\left[1+I_{\emptyset}\left(p^{o}\right) n^{c}\right]\right\}\right. \\
\left.\left.\left[-\frac{1}{2} V_{\emptyset}\left(p^{o}\right)+\frac{1}{2} V_{c}\left(p^{o}\right) n^{c}-\frac{1}{5} V_{c d}\left(p^{o}\right) n^{c} n^{d}\right]\right\}\right\} .
\end{gathered}
$$

We recall that, according to the notation of section 1.7.2, $I_{a_{\ell}}^{\prime}\left(p^{o}\right) \equiv p^{o} \frac{\partial}{\partial p^{o}} I_{a_{\ell}}\left(p^{o}\right)$. We see clearly by examining the antisymmetric part of the Thomson and recoil terms, i.e. the terms proportional to $\mathrm{i} \epsilon_{a b c} n^{c}$, that the circular polarization is not excited and thus remains null if it is initially so. From now on, we will discard the antisymmetric term in the polarization tensor and in the Boltzmann equation. Additionally, since there is a small amount of energy transferred from the electrons to the photons in the recoil term $C_{a b, \mathrm{R}}$, there is also energy transferred from the photons to the electrons, and it is incompatible with a cold distribution of electrons. In order to have a consistent treatment of the collision term, we can still work in the electrons rest frame but we have at least to consider a thermal distribution of electrons. Consequently we turn to this case in the next section, but the results obtained for a cold distribution of electrons can be used to derive the collision tensor for this more general case.

\subsection{Thermal distribution of electrons with no bulk velocity}

If the distribution of the velocities of free electrons is thermal then we can apply the previous method for each of the electrons. That is for each momentum $\mathbf{q}$ in the integral on distribution function $g_{\mathrm{e}}(\mathbf{q})$, we choose a tetrad field $\tilde{\mathbf{e}}_{a}$ whose timelike vector $\tilde{\mathbf{e}}_{o}$ is aligned with this momentum $\left(\tilde{\mathbf{e}}_{o}=\mathbf{q} / m_{\mathrm{e}}\right)$. We then calculate the collision rate per electron defined in equations (6.15) and (6.16) in this frame [that is $\sigma_{\mathrm{T}} \tilde{n}_{\mathrm{e}} \tilde{C}_{\tilde{a} \tilde{b}, \mathrm{~T}}\left(p^{\tilde{c}}\right)$ and $\left.\sigma_{\mathrm{T}} \tilde{n}_{\mathrm{e}} \tilde{C}_{\tilde{a} \tilde{b}, \mathrm{R}}\left(p^{\tilde{c}}\right)\right]$, and since these quantities transform similarly to equation (1.46), then the collision term is expressed by

$C_{a b}\left(p^{h}\right)=\sigma_{\mathrm{T}} \int \frac{\mathrm{d}^{3} q^{i}}{(2 \pi)^{3} q^{o}} m_{\mathrm{e}} g_{\mathrm{e}}\left(q^{h}\right) S_{a}^{\tilde{c}} S_{b}^{\tilde{d}}\left[\tilde{\mathcal{C}}_{\tilde{c} \tilde{d}, \mathrm{~T}}\left(p^{\tilde{h}}\right)+\tilde{\mathcal{C}}_{\tilde{c} \tilde{d}, \mathrm{R}}\left(p^{\tilde{h}}\right)\right]$.

In practice this means that we need to use the transformation rules of the radiation multipoles, of the energy and of the momentum direction, in order to express everything inside this integral in function of the multipoles taken in the fundamental frame. The interest lies in the fact that integrals on the electron distribution function 
with odd powers of $\mathbf{v}$ (the velocity of the electron considered) vanish. Since we need only to go up to third order in the thermal velocities, then in that case we can restrict to second order in thermal velocity.

However we have many reasons to follow a much simpler track. First the terms coming from the second order change of frame (that is second order in $\mathbf{v}$ ) will lead after integration on the electron momentum to terms having a factor at least $T_{\mathrm{e}} / m_{\mathrm{e}} \equiv\left(\bar{T}+\delta T_{\mathrm{e}}\right) / m_{\mathrm{e}}$, and following the discussion of section 6.2. this factor can be comparable to first order perturbations in the metric $\left(\bar{T} / m_{\mathrm{e}}\right)$ or second order $\left(\delta T_{\mathrm{e}} / m_{\mathrm{e}}\right)$. Since we stop our expansion at second order, we can have in each of this term either a quantity whose smallest non-vanishing perturbation is the first order $\left(I_{\underline{a} \ell}\right.$ for $\left.\ell \geq 1\right)$ or a quantity whose smallest non-vanishing perturbation is the background $\left(I_{\emptyset}\right)$. If it is the latter case, then the term contributes to the famous Kompaneets collision term [85]. If it is the former case, then the term contributes to what can considered as corrections to the Kompaneets term for anisotropic radiation, but since it is linear in first order perturbations, this type of term cannot contribute to the bispectrum [44]. Similarly, the terms coming from the recoil term have an overall factor of $p^{\tilde{o}} / m_{\mathrm{e}}$ which is typically of order $T_{\mathrm{r}} / m_{\mathrm{e}}$, and for these terms the same reasoning applies. Consequently, when interested in the bispectrum, we should in principle just keep the Kompaneets term. However the expression of this famous Kompaneets collision term which is contained in the total collision term (6.19) is

$C_{c d, K}\left(p^{h}\right)=n_{\mathrm{e}} \sigma_{\mathrm{T}} \frac{S_{c d}}{2} \frac{1}{m_{\mathrm{e}} p^{o}} \frac{\partial}{\partial p^{o}}\left\{\left(p^{o}\right)^{4}\left[T_{\mathrm{e}} \frac{\partial I_{\emptyset}\left(p^{o}\right)}{\partial p^{o}}+I_{\emptyset}\left(p^{o}\right)\left(1+\frac{1}{2} I_{\emptyset}\left(p^{o}\right)\right)\right]\right\}$,

and it can be checked that for $I_{\emptyset}\left(p^{\circ}\right) / 2$ following a Bose-Einstein distribution of temperature $T_{\mathrm{r}}=T_{\mathrm{e}}$, then the Kompaneets collision term exactly vanishes, and if these two temperatures are close, as it is the case when recombination occurs, then it is of order $\left(T_{\mathrm{r}}-T_{\mathrm{e}}\right) / m_{\mathrm{e}}$. The Kompaneets contribution, which is at least a second order quantity in the sense of the discussion in section 6.2 bears thus only a linear response in the (primordial) first order metric perturbations through $\bar{T} / m_{\mathrm{e}}\left(T_{\mathrm{r}}^{(1)}-T_{\mathrm{e}}^{(1)}\right) / \bar{T}$. Consequently it cannot contribute to the bispectrum generated by evolution, and we will ignore it in the rest of this paper. Note also that on the form (6.20) of the Kompaneets collision term, it is obvious that $C_{c d, K}^{\#} \equiv a C_{c d, K} / p^{o}$ does conserve the photons number density [2, 46] and not the energy density (it is known to induce spectral distortions) as it is not the case on the expression (4.38) given in [48. Hence, the integrated counterpart of the Kompaneets term, $\mathcal{C}_{a b}^{\#}$, does not vanish and it is only because we focus on the bispectrum that we may discard it.

Gathering all these remarks, we conclude that for a thermal distribution of electrons with no bulk velocity, the collision term is given by

$$
C_{a b}\left(p^{c}\right)=n_{\mathrm{e}} \sigma_{\mathrm{T}} S_{a}^{c} S_{b}^{d} \mathcal{C}_{c d, \mathrm{~T}}\left(p^{c}\right)+\mathrm{O}(1)\left[\mathrm{O}\left(T_{\mathrm{e}} / m_{\mathrm{e}}\right)+\mathrm{O}\left(p^{o} / m_{\mathrm{e}}\right)\right],(6.2
$$

and that the terms of order $\mathrm{O}\left(T_{\mathrm{e}} / m_{\mathrm{e}}\right)$ and $\mathrm{O}\left(p^{o} / m_{\mathrm{e}}\right)$ are irrelevant for the purpose of computing the bispectrum generated by evolution. This method which consists in working in the baryons rest frame simplifies the physical interpretation of the collision tensor. Indeed on the form (6.17) and (6.21) we clearly see that in this frame in the tight coupling limit (that is when $\left.C_{a b, \mathrm{~T}}=0\right)$ there is no polarization $\left(P_{a b}=0\right)$ and the radiation is moving with the baryons as if it was a single fluid since the only nonvanishing multipole is $\mathcal{I}_{\emptyset}$. This terminates rigorously the discussion in section II.C.2 of 44. (see also the discussion at the end of section 6.6). 


\subsection{Thermal distribution with bulk velocity}

If the distribution of electrons has indeed a bulk velocity in the cosmological frame, then we just need to transform the result obtained in the baryons rest frame to the cosmological frame, and in this process, we keep only the terms which can contribute up to second order when performing perturbations. For the sake of comparison with literature, we only report the intensity part of the collision term and present here $C^{\#}$ rather than $C$. We obtain

$$
\begin{aligned}
S^{a b} C_{a b}^{\#}\left(p^{h}\right)=\tau^{\prime}\{ & {\left[-I\left(p^{o}, n^{i}\right)+I_{\emptyset}\left(p^{o}\right)+\frac{1}{10} I_{c d}\left(p^{o}\right) n^{c} n^{d}\right](1-\mathbf{n} \cdot \mathbf{v}) } \\
& -I_{\emptyset}^{\prime}\left(p^{o}\right) \mathbf{n} \cdot \mathbf{v}+\frac{7}{10} I_{c}\left(p^{o}\right) v^{c}+\frac{3}{10} I_{c}^{\prime}\left(p^{o}\right) v^{c}+\frac{2}{10} I_{c d}\left(p^{o}\right) n^{c} n^{d} \mathbf{n} \cdot \mathbf{v} \\
& -\frac{1}{10} I_{c d}^{\prime}\left(p^{o}\right) n^{c} n^{d} \mathbf{n} \cdot \mathbf{v}-\frac{1}{10} I_{c}\left(p^{o}\right) n^{c} \mathbf{n} \cdot \mathbf{v}+\frac{1}{10} I_{c}^{\prime}\left(p^{o}\right) n^{c} \mathbf{n} \cdot \mathbf{v} \\
& -\frac{1}{5} I_{c d}\left(p^{o}\right) n^{c} v^{d}+\frac{6}{35} I_{c d h}\left(p^{o}\right) n^{c} n^{h} v^{d}+\frac{3}{70} I_{c d h}^{\prime}\left(p^{o}\right) n^{c} n^{h} v^{d} \\
& +I_{\emptyset}^{\prime}\left(p^{o}\right) \mathbf{v} \cdot \mathbf{v}+\frac{3}{20} I_{\emptyset}^{\prime \prime}\left(p^{o}\right) \mathbf{v} \cdot \mathbf{v}+\frac{11}{20} I_{\emptyset}^{\prime \prime}\left(p^{o}\right)(\mathbf{n} \cdot \mathbf{v})^{2}+I_{\emptyset}^{\prime}\left(p^{o}\right)(\mathbf{n} \cdot \mathbf{v})^{2} \\
& -\frac{3}{5} E_{c d}\left(p^{o}\right) n^{c} n^{d}(1+\mathbf{v} \cdot \mathbf{n})+\frac{3}{5} E_{c d}^{\prime}\left(p^{o}\right) n^{c} n^{d} \mathbf{v} \cdot \mathbf{n}+\frac{6}{5} E_{c d}\left(p^{o}\right) n^{c} v^{d} \\
& -\frac{4}{7} E_{c d h}\left(p^{o}\right) n^{c} n^{d} v^{h}-\frac{1}{7} E_{c d h}^{\prime}\left(p^{o}\right) n^{c} n^{d} v^{h} \\
& \left.+\frac{2}{5} B_{c d}\left(p^{o}\right) \epsilon^{f h d} v_{h} n_{f} n^{c}+\frac{2}{5} B_{c d}^{\prime}\left(p^{o}\right) \epsilon^{f h d} v_{h} n_{f} n^{c}\right\}+\mathrm{O}\left(T_{\mathrm{e}} / m_{\mathrm{e}}, p^{o} / m_{\mathrm{e}}\right),
\end{aligned}
$$

where $\tau^{\prime} \equiv a n_{\mathrm{e}} \sigma_{\mathrm{T}} \delta$.

Though we have also obtained the full collision term for the polarization part, we only report here the result of its energy integrated counterpart, in order to simplify the expressions obtained.

$$
\begin{aligned}
\mathcal{C}_{a b}^{\#}\left(n^{i}\right)=\tau^{\prime} \frac{1}{2} S_{a b}[ & \left(-\mathcal{I}\left(n^{i}\right)+\mathcal{I}_{\emptyset}+\frac{1}{10} \mathcal{I}_{c d} n^{c} n^{d}\right)(1-\mathbf{n} \cdot \mathbf{v}) \\
& -\frac{1}{2} \mathcal{I}_{c} v^{c}-\frac{1}{2} \mathcal{I}_{c} n^{c} \mathbf{n} \cdot \mathbf{v}+\frac{3}{5} \mathcal{I}_{c d} n^{c} n^{d} \mathbf{n} \cdot \mathbf{v} \\
& -\frac{1}{5} \mathcal{I}_{c d} n^{c} v^{d}+4 \mathcal{I}_{\emptyset} \mathbf{n} . \mathbf{v}-\mathcal{I}_{\emptyset} \mathbf{v} \cdot \mathbf{v}+7 \mathcal{I}_{\emptyset}(\mathbf{v} \cdot \mathbf{n})^{2} \\
& \left.-\frac{3}{5} \mathcal{E}_{c d} n^{c} n^{d}(1+5 \mathbf{v} \cdot \mathbf{n})+\frac{6}{5} \mathcal{E}_{c d} n^{c} v^{d}-\frac{6}{5} \mathcal{B}_{c d} \epsilon^{f h d} v_{h} n_{f} n^{c}\right] \\
+\tau^{\prime}[- & \mathcal{P}_{a b}\left(n^{i}\right)(1-\mathbf{n} \cdot \mathbf{v})+\left(-\frac{1}{10} \mathcal{I}_{a b}+\frac{3}{5} \mathcal{E}_{a b}\right)(1+3 \mathbf{v} \cdot \mathbf{n}) \\
+ & \left.\frac{6}{5} \mathcal{E}_{a c} n^{c} v_{b}-\frac{1}{5} \mathcal{I}_{a c} n^{c} v_{b}+\frac{6}{5} \mathcal{B}_{a}{ }^{d} \epsilon_{b h d} v^{h}+\frac{1}{2} \mathcal{I}_{a} v_{b}-\mathcal{I}_{\emptyset} v_{a} v_{b}\right]^{\mathrm{TT}} \\
+\mathcal{O}( & \left.T_{\mathrm{e}} / m_{\mathrm{e}}, p^{o} / m_{\mathrm{e}}\right) .
\end{aligned}
$$

$\S$ Note here that $n_{\mathrm{e}}$ is the electron number density as seen in the electrons rest frame. Though we have changed the frame for expressing the collision term we keep this definition for the electron number density since this is the most physical. However, a change of frame only affects the number density by a factor $\gamma \simeq 1+\mathbf{v} \cdot \mathbf{v} / 2$, and since the background collision term vanishes, the second order perturbation of the collision term is not affected by the precise choice of frame used to define the electron number density. 
The trace part of this energy-integrated collision term as well as its non-energy integrated counterpart which is given in equation (6.22) reproduce the results obtained previously in [46, 48, 86] for unpolarized radiation.

\subsection{Multipole decomposition}

This last expression can then be decomposed in multipoles, in order to separate the intensity from the linear polarization part.

$$
\begin{aligned}
\frac{1}{\tau^{\prime}} \mathcal{C}^{\#}[\mathcal{I}]_{\underline{a_{\ell}}}= & -\mathcal{I}_{\underline{a_{\ell}}}+\frac{(\ell+1)}{(2 \ell+3)} \mathcal{I}_{\underline{a_{\ell}} b} v^{b}+\mathcal{I}_{\left\langle\underline{a_{\ell-1}}\right.} v_{\left.a_{\ell}\right\rangle}+\delta_{\ell}^{0}\left[\frac{4}{3} \mathcal{I}_{\emptyset} v_{b} v^{b}-\frac{2}{3} \mathcal{I}_{b} v^{b}+\mathcal{I}_{\emptyset}\right] \\
& +\delta_{\ell}^{1}\left[3 \mathcal{I}_{\emptyset} v_{a_{1}}\right]+\delta_{\ell}^{3}\left[\frac{1}{2} \mathcal{I}_{\left\langle a_{1} a_{2}\right.} v_{\left.a_{3}\right\rangle}-3 \mathcal{E}_{\left\langle a_{1} a_{2}\right.} v_{\left.a_{3}\right\rangle}\right] \\
& +\delta_{\ell}^{2}\left[\frac{1}{10} \mathcal{I}_{a_{1} a_{2}}-\frac{3}{5} \mathcal{E}_{a_{1} a_{2}}-\frac{1}{2} \mathcal{I}_{\left\langle a_{1}\right.} v_{\left.a_{2}\right\rangle}+7 \mathcal{I}_{\emptyset} v_{\left\langle a_{1}\right.} v_{\left.a_{2}\right\rangle}-\frac{6}{5} \epsilon_{b c\left\langle a_{1}\right.} v^{b} \mathcal{B}_{\left.a_{2}\right\rangle}^{c}\right] \\
\frac{1}{\tau^{\prime}} \mathcal{C}^{\#}[\mathcal{E}]_{\underline{a_{\ell}}}= & -\mathcal{E}_{a_{\ell}}+\frac{(\ell-1)(\ell+3)}{(2 \ell+3)(\ell+1)} \mathcal{E}_{a_{\ell} b} v^{b}+\mathcal{E}_{\left\langle a_{\ell-1}\right.} v_{\left.a_{\ell}\right\rangle} \\
& +\delta_{\ell}^{3}\left[-\frac{1}{2} \mathcal{I}_{\left\langle a_{1} a_{2}\right.} v_{\left.a_{3}\right\rangle}+3 \mathcal{E}_{\left\langle a_{1} a_{2}\right.} v_{\left.a_{3}\right\rangle}\right]-\frac{2}{\ell+1} \epsilon_{b c\left\langle a_{\ell}\right.} v^{b} \mathcal{B}_{\left.a_{\ell-1}\right\rangle}^{c} \\
& +\delta_{\ell}^{2}\left[-\frac{1}{10} \mathcal{I}_{a_{1} a_{2}}+\frac{3}{5} \mathcal{E}_{a_{1} a_{2}}+\frac{1}{2} \mathcal{I}_{\left\langle a_{1}\right.} v_{\left.a_{2}\right\rangle}-\mathcal{I}_{\emptyset} v_{\left\langle a_{1}\right.} v_{\left.a_{2}\right\rangle}+\frac{6}{5} \epsilon_{b c\left\langle a_{1}\right.} v^{b} \mathcal{B}_{\left.a_{2}\right\rangle}^{c}\right] \\
\frac{1}{\tau^{\prime}} \mathcal{C}^{\#}[\mathcal{B}]_{\underline{a_{\ell}}}= & -\mathcal{B}_{\underline{a_{\ell}}}+\frac{(\ell-1)(\ell+3)}{(2 \ell+3)(\ell+1)} \mathcal{B}_{a_{\ell} b} v^{b}+\mathcal{B}_{\left\langle\underline{a_{\ell-1}}\right.} v_{\left.a_{\ell}\right\rangle} \\
& +\frac{2}{\ell+1} \epsilon_{b c\left\langle a_{\ell}\right.} v^{b} \mathcal{E}_{\underline{\left.a_{\ell-1}\right\rangle}}^{c}+\delta_{\ell}^{2}\left[\frac{4}{5} \epsilon_{b c\left\langle a_{1}\right.} v^{b} \mathcal{E}_{\left.a_{2}\right\rangle}^{c}-\frac{2}{15} \epsilon_{b c\left\langle a_{1}\right.} v^{b} \mathcal{I}_{\left.a_{2}\right\rangle}^{c}\right]
\end{aligned}
$$

We recall that the multipoles are projected so all indices $a, b, c \ldots$ in the above expressions could in fact be replaced by $i, j, k \ldots$..

In the case where there is no polarization, the expression 6.24 for the intensity part of the collision is nearly consistent with equations (64-67) of [57. Indeed the only discrepancy is the coefficient in front of $\mathcal{I}_{\emptyset} v_{\left\langle a_{1}\right.} v_{\left.a_{2}\right\rangle}$, which in our case is 7 and in the case of [57] is 3. In their notation, this discrepancy arises from a missing factor 6 in their equation (60) in front of the factor $\left(v_{B}^{c} e_{c}\right)^{2}$. The terms in the first bracket of the r.h.s in this equation can be traced directly to the expansion of $\left[\gamma_{B}\left(1-v_{B}^{c} e_{c}\right)\right]^{-3}$, arising from the change of frame between the baryons rest frame and the fundamental frame. Indeed, in the definition 4.6 applied to the collision tensor, $C[]$ is a scalar but (noting here $\tilde{\mathbf{e}}_{a}$ the tetrad in the baryons rest frame) $\left(p^{o}\right)^{3} \mathrm{~d} p^{o}=\left(p^{\tilde{o}}\right)^{3} \mathrm{~d} p^{\tilde{o}}\left[\gamma\left(1-n^{i} v_{i}\right)\right]^{-4}$, and in the definition 4.1 of $\mathcal{C}^{\#}$ there is an additional factor $1 / p^{o}=\gamma\left(1-n^{i} v_{i}\right) / p^{\tilde{o}}$. As a consequence, the factor -3 in front of the term $\rho_{R} v_{B}^{\langle a} v_{B}^{b\rangle}$ in equation (63) of [57] should be -7 .

In the tight coupled limit, that is when we can neglect (when compared to the Liouville operator) the r.h.s of equations (6.24]6.26), then it is obvious that

$$
\begin{aligned}
& \mathcal{E}_{\underline{i_{\ell}}}=\mathcal{B}_{\underline{i_{\ell}}}=\mathcal{I}_{i_{\underline{\ell}}}=0, \quad \text { if } \quad \ell \geq 3, \\
& \mathcal{E}_{i j}=\mathcal{B}_{i j}=0,
\end{aligned}
$$




$$
\begin{aligned}
& \frac{2}{15} \mathcal{I}_{i j}=T_{\langle i j\rangle}=\frac{4}{3} \mathcal{I}_{\emptyset} v_{\langle i} v_{j\rangle}, \\
& \mathcal{I}_{i}=4 \mathcal{I}_{\emptyset} v_{i} .
\end{aligned}
$$

We recover of course that since there is no polarization in the baryons rest frame, as noted at the end of section 6.4. then there is no polarization in any frame, and we check from the comparison with a perturbed stress-energy tensor with no anisotropic stress given in equations (5.28 5.31) that the multipoles $\mathcal{I}_{a}$ and $\mathcal{I}_{a b}$, which vanish in the baryons rest frame, are non-vanishing only because of the change of frame, and consequently in this tight coupled limit there is a quadrupole but no anisotropic stress 87.

\subsection{Perturbative expansion of the collision term}

Though this result might appear to be general since we have not performed a perturbative expansion around a FL space-time, it is not general since we have kept terms quadratic in the change of frame velocity $\mathbf{v}$ only when they multiply $\mathcal{I}_{\emptyset}$, since this is the only quantity which will have a background contribution. The full second order transformation (in $\mathbf{v}$ ) can be computed using the transformation rules at second order in $\mathbf{v}$ for the multipoles involved in the Thomson collision term (6.17) and we have reported them in Appendix A This full result at second order in the bulk velocity $\mathbf{v}$ has already been derived in [88, but without polarization. By developing with the expansion (2.4) the intensity, the electric and the magnetic multipoles along with $\tau^{\prime}$ and $\mathbf{v}$, we can perform a perturbative expansion of the collision term following the same notations as for the Liouville operator expansion, that is as in equation (4.5). The background collision term vanishes. Since we have decided to discard the first order magnetic multipole of radiation (because we can neglect first order vector and tensor perturbations), there is no contribution to the magnetic multipole of the collision term. The first order intensity and electric multipoles read

$$
\begin{aligned}
& \mathcal{C}^{\#(1)}\left[\overline{\mathcal{I}}, \hat{\mathcal{I}}^{(1)}\right]_{\underline{a_{\ell}}}=\bar{\tau}^{\prime}\left\{-\hat{\mathcal{I}}_{\underline{a_{\ell}}}^{(1)}+\delta_{\ell}^{0} \hat{\mathcal{I}}_{\emptyset}^{(1)}+\delta_{\ell}^{1} 4 \overline{\mathcal{I}}_{\emptyset} \hat{v}_{a_{1}}^{(1)}+\delta_{\ell}^{2}\left[\frac{1}{10} \hat{\mathcal{I}}_{a_{1} a_{2}}^{(1)}-\frac{3}{5} \hat{\mathcal{E}}_{a_{1} a_{2}}^{(1)}\right]\right\}, \\
& \mathcal{C}^{\#(1)}\left[\hat{\mathcal{E}}^{(1)}\right]_{\underline{a_{\ell}}}=\bar{\tau}^{\prime}\left\{-\hat{\mathcal{E}}_{\underline{a_{\ell}}}^{(1)}+\delta_{\ell}^{2}\left[-\frac{1}{10} \hat{\mathcal{I}}_{a_{1} a_{2}}^{(1)}+\frac{3}{5} \hat{\mathcal{E}}_{a_{1} a_{2}}^{(1)}\right]\right\} .
\end{aligned}
$$

At second order, the linear contribution is given by

$$
\begin{aligned}
& \mathcal{C}^{\#(2)}\left[\overline{\mathcal{I}}, \hat{\mathcal{I}}^{(2)}\right]_{\underline{a_{\ell}}}={\overline{\tau^{\prime}}}^{\prime}\left\{-\hat{\mathcal{I}}_{\underline{a_{\ell}}}^{(2)}+\delta_{\ell}^{0} \hat{\mathcal{I}}_{\emptyset}^{(2)}+\delta_{\ell}^{1} 4 \overline{\mathcal{I}}_{\emptyset} \hat{v}_{a_{1}}^{(2)}+\delta_{\ell}^{2}\left[\frac{1}{10} \hat{\mathcal{I}}_{a_{1} a_{2}}^{(2)}-\frac{3}{5} \hat{\mathcal{E}}_{a_{1} a_{2}}^{(2)}\right]\right\}, \\
& \mathcal{C}^{\#(2)}\left[\hat{\mathcal{E}}^{(2)}\right]_{\underline{a_{\ell}}}=\overline{\tau^{\prime}}\left\{-\hat{\mathcal{E}}_{\underline{a_{\ell}}}^{(2)}+\delta_{\ell}^{2}\left[-\frac{1}{10} \hat{\mathcal{I}}_{a_{1} a_{2}}^{(2)}+\frac{3}{5} \hat{\mathcal{E}}_{a_{1} a_{2}}^{(2)}\right]\right\}, \\
& \mathcal{C}^{\#(2)}\left[\hat{\mathcal{B}}^{(2)}\right]_{\underline{a_{\ell}}}=-\bar{\tau}^{\prime} \hat{\mathcal{B}}_{\underline{a_{\ell}}}^{(2)} .
\end{aligned}
$$

As for the quadratic contribution, it is given by

$$
\begin{aligned}
\mathcal{C}^{\#(1)(1)}\left[\overline{\mathcal{I}}, \hat{\mathcal{I}}^{(1)}\right]_{\underline{a_{\ell}}}= & 2 \bar{\tau}^{\prime}\left\{\frac{(\ell+1)}{(2 \ell+3)} \hat{\mathcal{I}}_{a_{\ell} b}^{(1)} \hat{v}^{b(1)}+\hat{\mathcal{I}}_{\left\langle a_{\ell-1}\right.}^{(1)} \hat{v}_{\left.a_{\ell}\right\rangle}^{(1)}+\delta_{\ell}^{1} 3 \hat{\mathcal{I}}_{\emptyset}^{(1)} \hat{v}_{a_{1}}^{(1)}\right. \\
& +\delta_{\ell}^{0}\left[\frac{4}{3} \overline{\mathcal{I}}_{\emptyset} \hat{v}_{b}^{(1)} \hat{v}^{b(1)}-\frac{2}{3} \hat{\mathcal{I}}_{b}^{(1)} \hat{v}^{b(1)}\right]+\delta_{\ell}^{2}\left[-\frac{1}{2} \hat{\mathcal{I}}_{\left\langle a_{1}\right.}^{(1)} \hat{v}_{\left.a_{2}\right\rangle}^{(1)}+7 \overline{\mathcal{I}}_{\emptyset} \hat{v}_{\left\langle a_{1}\right.}^{(1)} \hat{v}_{\left.a_{2}\right\rangle}^{(1)}\right] \\
& \left.+\delta_{\ell}^{3}\left[\frac{1}{2} \hat{\mathcal{I}}_{\left\langle a_{1} a_{2}\right.}^{(1)} \hat{v}_{\left.a_{3}\right\rangle}^{(1)}-3 \mathcal{E}_{\left\langle a_{1} a_{2}\right.}^{(1)} \hat{v}_{\left.a_{3}\right\rangle}^{(1)}\right]\right\}+2{\hat{\tau^{\prime}}}^{(1)} \mathcal{C}^{\#(1)}\left[\overline{\mathcal{I}}, \hat{\mathcal{I}}^{(1)}\right]_{\underline{a_{\ell}}},
\end{aligned}
$$




$$
\begin{aligned}
& \mathcal{C}^{\#(1)(1)}\left[\hat{\mathcal{E}}^{(1)}\right]_{\underline{a_{\ell}}}=2{\overline{\tau^{\prime}}}\left\{\frac{(\ell-1)(\ell+3)}{(2 \ell+3)(\ell+1)} \hat{\mathcal{E}}_{\underline{a \ell}_{\underline{b}}}^{(1)} \hat{v}^{b(1)}+\hat{\mathcal{E}}_{\left\langle\underline{a_{\ell-1}}\right.}^{(1)} \hat{v}_{\left.a_{\ell}\right\rangle}^{(1)}\right. \\
& +\delta_{\ell}^{3}\left[-\frac{1}{2} \hat{\mathcal{I}}_{\left\langle a_{1} a_{2}\right.}^{(1)} \hat{v}_{\left.a_{3}\right\rangle}^{(1)}+3 \hat{\mathcal{E}}_{\left\langle a_{1} a_{2}\right.}^{(1)} \hat{v}_{\left.a_{3}\right\rangle}^{(1)}\right]-\frac{2}{\ell+1} \epsilon_{b c\left\langle a_{\ell}\right.} \hat{v}^{b(1)} \hat{\mathcal{B}}_{\underline{\left.a_{\ell-1}\right\rangle}}^{c(1)} \\
& \left.+\delta_{\ell}^{2}\left[\frac{1}{2} \hat{\mathcal{I}}_{\left\langle a_{1}\right.}^{(1)} \hat{v}_{\left.a_{2}\right\rangle}^{(1)}-\overline{\mathcal{I}}_{\emptyset} \hat{v}_{\left\langle a_{1}\right.}^{(1)} \hat{v}_{\left.a_{2}\right\rangle}^{(1)}\right]\right\}+2{\hat{\tau^{\prime}}}^{(1)} \mathcal{C}^{\#(1)}\left[\hat{\mathcal{E}}^{(1)}\right]_{\underline{a_{\ell}}}, \\
& \mathcal{C}^{\#(1)(1)}\left[\hat{\mathcal{B}}^{(1)}\right]_{\underline{a_{\ell}}}=2 \bar{\tau}^{\prime}\left\{\frac{2}{\ell+1} \epsilon_{b c\left\langle a_{\ell}\right.} \hat{v}^{b(1)} \hat{\mathcal{E}}^{c(1)} \underline{\left.a_{\ell-1}\right\rangle}\right. \\
& \left.+\delta_{\ell}^{2}\left[\frac{4}{5} \epsilon_{b c\left\langle a_{1}\right.} \hat{v}^{b(1)} \mathcal{E}_{\left.a_{2}\right\rangle}^{c(1)}-\frac{2}{15} \epsilon_{b c\left\langle a_{1}\right.} \hat{v}^{b(1)} \hat{\mathcal{I}}^{c(1)}{ }_{\left.a_{2}\right\rangle}\right]\right\} .
\end{aligned}
$$

\subsection{The fluid equations for baryons}

The total stress-energy tensor is conserved

$$
\nabla_{\mu} T_{\mathrm{b}+\mathrm{r}}^{\mu \nu}=\nabla_{\mu} T_{\mathrm{b}}^{\mu \nu}+\nabla_{\mu} T_{\mathrm{r}}^{\mu \nu}=0 .
$$

This arises from the Bianchi identities, and thus the action of baryons on photons is opposite to the action of photons on baryons. This means that we can define the force resulting from the action of photons on baryons and the force resulting from the action of baryons on photons by

$$
\nabla_{\mu} T_{\mathrm{b}}^{\mu \nu} \equiv F_{\mathrm{r} \rightarrow \mathrm{b}}^{\nu}=-\nabla_{\mu} T_{\mathrm{r}}^{\mu \nu} \equiv-F_{\mathrm{b} \rightarrow \mathrm{r}}^{\nu}
$$

The expression of the force in the tetrad basis is further given by

$$
F_{\mathrm{r} \rightarrow \mathrm{b}}^{a}=\frac{1}{a} \int q^{a} C^{\#}[g]\left(q^{i}\right) \frac{\mathrm{d}^{3} q^{i}}{(2 \pi)^{3}}=-\frac{1}{a} \int N^{a} \mathcal{C}^{\#}[I]\left(n^{i}\right) \frac{\mathrm{d}^{2} \Omega}{4 \pi},
$$

and from equations (5.13) and (1.59), its components are easily related to the moments of the radiation collision term by

$$
\begin{aligned}
& a F_{\mathrm{r} \rightarrow \mathrm{b}}^{o}=-\mathcal{C}^{\#}[I]^{\emptyset}, \\
& a F_{\mathrm{r} \rightarrow \mathrm{b}}^{i}=-\frac{1}{3} \mathcal{C}^{\#}[I]^{i} .
\end{aligned}
$$

It is then straightforward to deduce the perturbative expansion of the force, which is inherited directly from the perturbative expansion of the radiation collision term. Note that in particular $F_{\mathrm{r} \rightarrow \mathrm{b}}^{o(1)}=0$.

\section{From PSTF multipoles to normal modes components}

\subsection{The normal modes in Fourier space}

The equations (4.25,4.24), 44.314.34), (5.34 5.36) with equations (6.30 6.35) and (6.39) are a key result of this paper, since they provide the full second order Boltzmann hierarchy for the coupled system of baryons and photons. However in order to make contact with the standard approach of $\mathrm{CMB}$ numerical integration, it is more convenient to describe the angular dependence of the radiation functions using the normal modes components [15. And in order to perform a numerical integration in the most simple way, we also turn to Fourier space in order to solve only for a time 
integral. It has been shown 89 that we can convert the PSTF multipoles into normal modes components by decomposing the quantities $\mathcal{I}, \mathcal{E}$ and $\mathcal{B}$ according to

$\mathcal{X}\left(x^{A}, n^{i}\right)=\sum_{\ell m} \int \frac{\mathrm{d}^{3} \mathbf{k}}{(2 \pi)^{3 / 2}} \mathcal{X}_{\ell}^{m}(\mathbf{k}, \eta) G_{\ell m}^{\mathcal{X}}\left(\mathbf{k}, x^{I}, n^{j}\right)=\sum_{\ell=0}^{\infty} \mathcal{X}_{a_{\ell}}\left(x^{A}\right) n \underline{\left\langle\underline{a_{\ell}}\right\rangle}$,

where $\mathcal{X}$ stands for $\mathcal{I}, \mathcal{E}$ or $\mathcal{B}$. Here we have used the notation $\mathbf{k}$, that is a boldface font, for a mode in the Fourier transform though it is only a three dimensional vector. The modes $G_{\ell m}^{\mathcal{X}}\left(n^{i}\right)$ according to which we decompose are defined by

$$
\begin{aligned}
& G_{\ell m}^{\mathcal{I}}\left(\mathbf{k}, x^{I}, \mathbf{n}\right)=\overline{\mathcal{I}} \frac{1}{N_{\ell}} e^{\mathrm{i} k_{I} x^{I}} Y^{\ell m}(\mathbf{n}), \\
& G_{\ell m}^{\mathcal{E}}\left(\mathbf{k}, x^{I}, \mathbf{n}\right)=-G_{\ell m}^{\mathcal{B}}\left(\mathbf{k}, x^{I}, \mathbf{n}\right)=\overline{\mathcal{I}} \frac{M_{\ell}}{\sqrt{2} N_{\ell}} e^{\mathrm{i} k_{I} x^{I}} Y^{\ell m}(\mathbf{n}),
\end{aligned}
$$

with

$$
N_{\ell} \equiv \mathrm{i}^{\ell} \sqrt{\frac{(2 \ell+1)}{4 \pi}} .
$$

The components $\mathcal{X}_{\ell m}(\mathbf{k}, \eta)$ are the time-dependent components in Fourier space of the spherical harmonics decomposition, also called normal modes. Note that we have also factorized the background intensity so these quantities are now dimensionless, but we did not divide by 4 as is usually done when we want to have a quantity which can be interpreted as a temperature. Instead we decompose $\mathcal{I} / \overline{\mathcal{I}}$ which is the fractional variation of the energy density of radiation as it is what is really measured. The $\mathcal{X}_{\ell m}(\mathbf{k}, \eta)$ can be obtained from the PSTF multipoles using the formulas

$$
\begin{aligned}
& \hat{\mathcal{I}}_{\ell}^{m}(\mathbf{k}, \eta)=\frac{1}{\overline{\mathcal{I}}} N_{\ell} \Delta_{\ell} \int \frac{\mathrm{d}^{3} x^{I}}{(2 \pi)^{3 / 2}} e^{-\mathrm{i} k_{I} x^{I}} \mathcal{Y}_{\ell \underline{m}}^{\star} \underline{a_{\ell}} \hat{\mathcal{I}}_{\underline{a_{\ell}}}\left(x^{A}\right), \\
& \hat{\mathcal{E}}_{\ell}^{m}(\mathbf{k}, \eta)=\frac{1}{\overline{\mathcal{I}}} \frac{N_{\ell} \sqrt{2}}{M_{\ell}} \Delta_{\ell} \int \frac{\mathrm{d}^{3} x^{I}}{(2 \pi)^{3 / 2}} e^{-\mathrm{i} k_{I} x^{I}} \mathcal{Y}_{\ell \underline{m}}^{\star} \underline{a_{\ell}} \hat{\mathcal{E}}_{\underline{a_{\ell}}}\left(x^{A}\right), \\
& \hat{\mathcal{B}}_{\ell}^{m}(\mathbf{k}, \eta)=-\frac{1}{\overline{\mathcal{I}}} \frac{N_{\ell} \sqrt{2}}{M_{\ell}} \Delta_{\ell} \int \frac{\mathrm{d}^{3} x^{I}}{(2 \pi)^{3 / 2}} e^{-\mathrm{i} k_{I} x^{I}} \mathcal{Y}_{\ell}^{\star} \frac{a_{\ell}}{\hat{\mathcal{B}}_{\underline{a}}}\left(x^{A}\right) .
\end{aligned}
$$

The functions $\mathcal{Y}_{\ell m}^{\frac{a_{\ell}}{\ell m}}$ are null if one of the indices in $\underline{a_{\ell}}$ is $o$, since it is already the case for the indices in the intensity, the electric and the magnetic multipoles. These quantities are built in details in [69, and in the case where $m \geq 0$ they are defined by

$$
\begin{aligned}
\mathcal{Y}_{\ell m}^{i_{\ell}}= & (-1)^{m}\left[\frac{2 \ell+1}{4 \pi} \frac{(\ell-m) !}{(\ell+m) !}\right]^{1 / 2} \sum_{j=0}^{[(\ell-m) / 2]} \frac{(-1)^{j}(2 \ell-2 j) !}{2^{\ell} j !(\ell-j) !(\ell-m-2 j) !} \\
& \times\left(\delta_{1}^{\left(i_{1}\right.}+\mathrm{i} \delta_{2}^{\left(i_{1}\right.}\right) \ldots\left(\delta_{1}^{i_{m}}+\mathrm{i} \delta_{2}^{i_{m}}\right) \delta_{3}^{i_{m+1}} \ldots \delta_{3}^{i_{\ell-2 j}} \delta^{i_{\ell-2 j+1} i_{\ell-2 j+2}} \ldots \delta^{i_{\ell-1} i_{\ell)}} .
\end{aligned}
$$

If $m<0$, then it is defined by $\mathcal{Y}_{\ell m}^{i_{\ell}}=(-1)^{m} \mathcal{Y}_{\ell}^{\star} \frac{i_{\ell}}{-m}$. The PSTF multipoles can be conversely obtained from the normal modes components by the inverse formulae

$$
\begin{aligned}
& \hat{\mathcal{E}}_{\underline{a_{\ell}}}\left(x^{A}\right)=\overline{\mathcal{I}} \frac{M_{\ell}}{\sqrt{2} N_{\ell}} \int \frac{\mathrm{d}^{3} \mathbf{k}}{(2 \pi)^{3 / 2}} e^{\mathrm{i} k_{I} x^{I}} \sum_{m=-\ell}^{\ell} \mathcal{Y}_{\underline{a_{\ell}}}^{\ell m} \hat{\mathcal{E}}_{\ell}^{m}(\mathbf{k}, \eta), \\
& \hat{\mathcal{I}}_{\underline{a_{\ell}}}\left(x^{A}\right)=\overline{\mathcal{I}} \frac{1}{N_{\ell}} \int \frac{\mathrm{d}^{3} \mathbf{k}}{(2 \pi)^{3 / 2}} e^{\mathrm{i} k_{I} x^{I}} \sum_{m=-\ell}^{\ell} \mathcal{Y}_{\underline{a_{\ell}}}^{\ell m} \hat{\mathcal{I}}_{\ell}^{m}(\mathbf{k}, \eta), \\
& \hat{\mathcal{B}}_{\underline{a_{\ell}}}\left(x^{A}\right)=-\overline{\mathcal{I}} \frac{M_{\ell}}{\sqrt{2} N_{\ell}} \int \frac{\mathrm{d}^{3} \mathbf{k}}{(2 \pi)^{3 / 2}} e^{\mathrm{i} k_{I} x^{I}} \sum_{m=-\ell}^{\ell} \underline{\mathcal{Y}}_{\underline{a}_{\ell}}^{\ell m} \hat{\mathcal{B}}_{\ell}^{m}(\mathbf{k}, \eta) .
\end{aligned}
$$




\subsection{The basis for the decomposition of tensors}

In order to decompose the metric perturbations and the fluid perturbations according to this spherical harmonics scheme, we start from the vectors

$\overline{\mathbf{e}}_{(0)} \equiv-\overline{\mathbf{e}}_{3}, \quad \overline{\mathbf{e}}_{(1)} \equiv \frac{1}{\sqrt{2}}\left(\overline{\mathbf{e}}_{1}+\mathrm{i} \overline{\mathbf{e}}_{2}\right), \quad \overline{\mathbf{e}}_{(-1)} \equiv-\frac{1}{\sqrt{2}}\left(\overline{\mathbf{e}}_{1}-\mathrm{i} \overline{\mathbf{e}}_{2}\right)$.

These are not true vectors since they live on the background space-time and their indices are raised and lowered by the euclidian metric $\delta_{I J}$ and its inverse $\delta^{I J}$. Their associated forms are thus

$\overline{\mathbf{e}}^{(0)} \equiv-\overline{\mathbf{e}}^{3}, \quad \overline{\mathbf{e}}^{(1)} \equiv \frac{1}{\sqrt{2}}\left(\overline{\mathbf{e}}^{1}+\mathrm{i}^{2}\right), \quad \overline{\mathbf{e}}^{(-1)} \equiv-\frac{1}{\sqrt{2}}\left(\overline{\mathbf{e}}^{1}-\mathrm{i}^{2}\right)$.

We then build a tensor basis in Fourier space out of this basis. We start from the scalar basis which in Fourier space is given by

$$
Q^{(0)} \equiv \exp \left(e^{\mathrm{i} k_{I} x^{I}}\right)
$$

and we align $\mathbf{k}$ with $\overline{\mathbf{e}}_{3}$. It is then used to build the scalar basis of higher rank tensors according to

$$
\begin{aligned}
& Q_{I}^{(0)} \equiv-\partial_{I} Q^{(0)} / k=\mathrm{i} \bar{e}_{I}^{(0)} Q^{(0)} \\
& Q_{I J}^{(0)} \equiv \partial_{\langle I} \partial_{J\rangle} Q^{(0)} / k^{2}=\left(-\bar{e}_{I}^{(0)} \bar{e}_{J}^{(0)}+\delta_{I J} / 3\right) Q^{(0)},
\end{aligned}
$$

since perturbation variables "live" on the background space-time. We then remark that

$$
Q^{(0)}=G_{00}^{\mathcal{I}}, \quad n^{i} Q_{I}^{(0)}=G_{10}^{\mathcal{I}}, \quad n^{i} n^{j} Q_{I J}^{(0)}=\frac{2}{3} G_{20}^{\mathcal{I}},
$$

and this enables us to make contact with the normal modes decomposition first performed for the directional dependence of radiation. For vectors, we follow the same method and we start from the basis

$$
Q_{I}^{( \pm 1)} \equiv \mathrm{i} \bar{e}_{I}^{( \pm 1)} Q^{(0)}
$$

from which we can build a basis for vector type perturbations of higher order rank tensors (we restrict to rank two tensors, since in the problem at hand we have no rank higher than 2)

$$
Q_{I J}^{( \pm 1)} \equiv-\partial_{(I} Q_{J)}^{( \pm 1)} / k=\mathrm{i} \bar{e}_{(I}^{(0)} Q_{J)}^{( \pm 1)}
$$

We then make contact with the normal modes decomposition by noting that

$$
n^{i} Q_{I}^{( \pm 1)}=G_{1 \pm 1}^{\mathcal{I}}, \quad n^{i} n^{j} Q_{I J}^{( \pm 1)}=\frac{1}{\sqrt{3}} G_{2 \pm 1}^{\mathcal{I}} .
$$

Note the difference in the definition of $Q_{I}^{ \pm 1}$ in equation (7.18) with respect to the expression given in [15]. However it agrees with the expression given in [90] up to factors $(-1)^{m}$ which arise because of a different convention in the spherical harmonics. Finally for tensors we use the basis

$$
Q_{I J}^{( \pm 2)} \equiv-\sqrt{\frac{3}{2}} \bar{e}_{I}^{ \pm 1} \bar{e}_{J}^{ \pm 1} Q^{(0)}
$$

which conveniently satisfies

$$
n^{i} n^{j} Q_{I J}^{ \pm 2}=G_{2 \pm 2}^{\mathcal{I}} .
$$


The (second order) vector perturbation of the metric is then decomposed as

$$
\hat{\Phi}_{I}^{(2)}\left(x^{A}\right)=\int \frac{\mathrm{d}^{3} \mathbf{k}}{(2 \pi)^{3 / 2}} \sum_{m= \pm 1} Q_{I}^{(m)}(\mathbf{k}) \hat{\Phi}_{m}^{(2)}(\mathbf{k}, \eta) .
$$

Similarly the (second order) tensor perturbations are decomposed according to

$$
\hat{H}_{I J}^{(2)}\left(x^{A}\right)=\int \frac{\mathrm{d}^{3} \mathbf{k}}{(2 \pi)^{3 / 2}} \sum_{m= \pm 1} Q_{I J}^{(2 m)}(\mathbf{k}) \hat{H}_{m}^{(2)}(\mathbf{k}, \eta) .
$$

As for gradients of the first order and second order scalar perturbations ( $\Phi$ and $\Psi$ ) they are decomposed similarly to the vector perturbations in

$$
\partial_{I} \hat{\Phi}\left(x^{A}\right)=\int \frac{\mathrm{d}^{3} \mathbf{k}}{(2 \pi)^{3 / 2}} \sum_{m=-1}^{1} Q_{I}^{(m)}(\mathbf{k}) k^{(m)} \Phi(\mathbf{k}, \eta) .
$$

This is equivalent to decomposing the Fourier mode $\mathbf{k}$ into a scalar part (in the direction $\overline{\mathbf{e}}_{(0)}$ ) and a vector part (in the plane orthogonal to $\overline{\mathbf{e}}_{(0)}$ ) according to

$$
k^{I}=k^{(0)} \bar{e}_{(0)}^{I}+k^{(1)} \bar{e}_{(1)}^{I}+k^{(-1)} \bar{e}_{(-1)}^{I}=\sum_{m=-1}^{1} k^{(m)} \bar{e}_{(m)}^{I} .
$$

The relation of these components to the Cartesian components of $\mathbf{k}$ are

$$
k^{(0)}=-k^{3}, \quad k^{(1)}=\frac{1}{\sqrt{2}}\left(k^{1}-\mathrm{i} k^{2}\right), \quad k^{(-1)}=-\frac{1}{\sqrt{2}}\left(k^{1}+\mathrm{i} k^{2}\right) \text {. }
$$

For the second order perturbations (or for first order perturbations when we solve the first order equations) we choose to align the mode $\mathbf{k}$ with $\overline{\mathbf{e}}_{(3)}$, which implies in the previous decomposition that $k^{(1)}=k^{(-1)}=0$, and $k^{(0)}=-k$. However for first order quantities appearing in quadratic terms in the second order equations, this is not possible because of the convolution generated by the Fourier transform. In the following we will also use the notation $\mathbb{k} \mathbf{k}=\mathbf{k} / k$ with the same decomposition as in equation (7.26).

As for the second order velocity of the electrons $\mathbf{v}$, it has a scalar degree of freedom and a vector degree of freedom according to the decomposition (2.6), and the Fourier components of this vector degree of freedom could be decomposed similarly to $\hat{\Phi}^{I}$, that is according to equation (7.23) with $m$ running from -1 to 1 . However since the velocity of the electrons always appear with tetrad basis components, it is not very convenient to go in the coordinate basis in order to split into scalar and vector modes and then transform back to tetrad components. We use instead for $v_{a}$ the same type of decomposition as we did for $\mathcal{I}_{a}$. Namely, this decomposition reads

$$
\hat{v}_{a}\left(x^{A}\right)=\frac{1}{N_{1}} \int \frac{\mathrm{d}^{3} \mathbf{k}}{(2 \pi)^{3 / 2}} e^{\mathrm{i} k_{I} x^{I}} \sum_{m=-1}^{1} \mathcal{Y}_{a}^{1 m} \hat{v}_{m}(\mathbf{k}, \eta) .
$$

\subsection{Comment on the SVT decomposition}

We have arbitrarily chosen to perform the multipole decomposition on $\hat{v}^{a}$ and not $\hat{V}^{A}$. If we use $\hat{V}^{A}$ for this multipole decomposition, then $\hat{V}_{0}$ matches the scalar component and $\hat{V}_{ \pm 1}$ match the vector components in the usual SVT decomposition of equation (2.6). We did not follow this approach for the baryons velocity, but we did

\| Note here that the hat denotes a unit vector and has nothing to do with a gauge invariant variable. 
it for the vector degrees of freedom in the metric $\hat{\Phi}^{I}$, and also for the tensor degrees of freedom $\hat{H}^{I J}$. We see from equation (5.41) that $\hat{v}^{a} \neq \hat{V}^{A}$ and consequently, when performing the multipole decomposition using $\hat{v}^{a}$ (which is what we have done in this paper) the normal modes components components are different $\left(\hat{v}_{m} \neq \hat{V}_{m}\right)$. It would thus be misleading to name $\hat{v}_{0}$ a scalar component and $\hat{v}_{ \pm 1}$ vector components. For the sake of the discussion here we will nonetheless use the terms coordinate $S V T$ and tetrad $S V T$ to name these different decompositions. Essentially the difference comes from the fact that when we perform the coordinate SVT decomposition of a vector, the decomposition is made on a Euclidian space-time which is the background space-time. Indeed the indices on the mode $k^{I}$ are lowered and raised by a simple $k_{I}=\delta_{I J} k^{I}$. The coordinate SVT decomposition of tensors is not motivated by physical reason but just by the Fourier transformation. On the other hand, in the tetrad SVT decomposition we use the tetrad indices $i$, that is we work in the locally Euclidian frame of the physical space-time, and indices are lowered and raised by $\hat{v}_{i}=\delta_{i j} \hat{v}^{j}$. The fictitious background Euclidian frame is different from the physical locally Euclidian frame. As it can be seen from equation (5.41), a local volume expansion higher than the average $(\Psi)$ leads to a difference in what is considered as a length, and the SVT components found in one decomposition are a rescaled version of those obtained in the other decomposition. If we had chosen not to neglect the first order gravitational waves, then the r.h.s of the second of the equations (5.41) would be supplemented by a term $2 E_{j}^{i(1)} V^{J(1)}$. Again, this would lead to a difference in the direction used to perform the decomposition and the scalar part of a vector in one approach would mix the scalar and the vector components of the other approach. Note that since we have neglected the first order vector and tensor perturbations of the metric, the decomposition of the second order vector and tensor perturbations of the metric in either approach leads to the same result. A more detailed discussion on the issue of SVT decomposition can be found in 61.

\subsection{Transforming the Boltzmann equation in normal modes components}

Since the second order equations are quadratic, we need to know how to compose the

$\mathcal{Y}_{a_{\ell}}^{\ell m}$. This is deduced from the composition rules of the spherical harmonics, and the key result is

$$
\begin{aligned}
\mathcal{Y}_{\left(i_{\ell_{1}}\right.}^{\ell_{1} m_{1}} \mathcal{Y}_{\underline{i_{\ell_{2}}} \ell_{2} m_{2}}^{\ell_{2}}=\sum_{\substack{\ell_{3}=0 \\
\left(\ell_{1}+\ell_{2}-\ell_{3}\right) \text { even }}}^{\ell_{1}+\ell_{2}} & \sqrt{\frac{\left(2 \ell_{1}+1\right)\left(2 \ell_{2}+1\right)}{4 \pi\left(2 \ell_{3}+1\right)}} C_{\ell_{1} m_{1} \ell_{2} m_{2}}^{\ell_{3}\left(m_{1}+m_{2}\right)} C_{\ell_{1} 0 \ell_{2} 0}^{\ell_{3} 0} \\
& \times \mathcal{Y}_{\underline{\left(i_{\ell_{3}}\right.}}^{\ell_{3}\left(m_{1}+m_{2}\right)} \delta_{i_{\ell_{3}+1} i_{\ell_{3}+2}} \ldots \delta_{\left.i_{\ell_{1}+\ell_{2}-1} i_{\ell_{1}+\ell_{2}}\right)},
\end{aligned}
$$

where the $C_{\ell_{1} m_{1} \ell_{2} m_{2}}^{\ell_{3} m_{3}}$ are the Clebsch-Gordan coefficients. A set of useful relations can be obtained by considering the cases where $\ell_{1}=\ell_{2}, \ell_{1}=\ell_{2}-1$ or $\ell_{1}=\ell_{2}+1$. These are reported in Appendix D.

\subsection{The Boltzmann hierarchy in normal modes components at first order}

Throughout this section we will use the following definitions that will simplify the notation

$$
{ }_{s}^{0} \uparrow K_{\ell}^{m} \equiv{ }_{s}^{0} \downarrow K_{\ell}^{m} \equiv \sqrt{\frac{\left(\ell^{2}-m^{2}\right)\left(\ell^{2}-s^{2}\right)}{\ell^{2}}},
$$




$$
\begin{aligned}
& { }_{s}^{ \pm 1} \uparrow K_{\ell}^{m} \equiv-\sqrt{\frac{(\ell \pm m)(\ell \pm m+1)\left(\ell^{2}-s^{2}\right)}{2 \ell^{2}}}, \\
& { }_{s}^{ \pm 1} \sqrt{ } K_{\ell}^{m} \equiv \sqrt{\frac{(\ell \pm m)(\ell \pm m-1)\left(\ell^{2}-s^{2}\right)}{2 \ell^{2}}}, \\
& { }^{0} \lambda_{\ell}^{m} \equiv-\frac{m}{\ell}, \quad{ }^{ \pm 1} \lambda_{\ell}^{m} \equiv \pm \frac{1}{\ell} \sqrt{\frac{(\ell \mp m+1)(\ell \pm m)}{2}} .
\end{aligned}
$$

At first order we choose to align the mode considered $\mathbf{k}$ with the direction $\overline{\mathbf{e}}_{3}$, and the dependence in $\mathbf{k}$ becomes only a dependence in its magnitude $k$. The set of equations obtained at first order is (dropping the obvious dependence of all quantities in $\eta$ in the rest of this paper)

$$
\begin{aligned}
& \mathcal{L}^{\#(1)}[\hat{\mathcal{I}}]_{\ell}^{0}(k)=\hat{\mathcal{I}}_{\ell}^{\prime 0}(k)+k\left[\frac{{ }_{0}^{0} \uparrow K_{\ell+1}^{0}}{2 \ell+3} \hat{\mathcal{I}}_{\ell+1}^{0}(k)-\frac{{ }_{0}^{0} \downarrow K_{\ell}^{0}}{2 \ell-1} \hat{\mathcal{I}}_{\ell-1}^{0}(k)\right] \\
& -\delta_{\ell}^{0} 4 \Psi^{\prime}(k)-\delta_{\ell}^{1} 4 k \hat{\Phi}(k), \\
& \mathcal{L}^{\#(1)}[\hat{\mathcal{E}}]_{\ell}^{0}(k)=\hat{\mathcal{E}}_{\ell}^{\prime 0}(k)+k\left[\frac{{ }_{2}^{0} \uparrow K_{\ell+1}^{0}}{2 \ell+3} \hat{\mathcal{E}}_{\ell+1}^{0}(k)-\frac{{ }_{2}^{0} \downarrow K_{\ell}^{0}}{2 \ell-1} \hat{\mathcal{E}}_{\ell-1}^{0}(k)\right] \text {, } \\
& \mathcal{C}^{\#(1)}[\hat{\mathcal{I}}]_{\ell}^{0}(k)=\bar{\tau}^{\prime}\left\{-\hat{\mathcal{I}}_{\ell}^{0}(k)+\delta_{\ell}^{0} \hat{\mathcal{I}}_{0}^{0}(k)+4 \delta_{\ell}^{1} \hat{v}_{0}(k)\right. \\
& \left.+\delta_{\ell}^{2} \frac{1}{10}\left[\hat{\mathcal{I}}_{2}^{0}(k)-\sqrt{6} \hat{\mathcal{E}}_{2}^{0}(k)\right]\right\}, \\
& \mathcal{C}^{\#(1)}[\hat{\mathcal{E}}]_{\ell}^{m}(k)=\bar{\tau}^{\prime}\left\{-\hat{\mathcal{E}}_{\ell}^{m}(k)-\delta_{\ell}^{2} \frac{\sqrt{6}}{10}\left[\hat{\mathcal{I}}_{2}^{0}(k)-\sqrt{6} \hat{\mathcal{E}}_{2}^{0}(k)\right]\right\} .
\end{aligned}
$$

As already mentionned, the first order magnetic modes are not excited since the first order vector and tensor modes are negligible, so we did not report them in the above equations. Additionally, the intensity and electric multipoles are only excited for $m=0$, that is the reason why we also only reported this case.

\subsection{The Boltzmann hierarchy in normal modes components at second order}

At second order, a Fourier mode $\mathbf{k}$ on a quadratic term will appear as a convolution on modes $\mathbf{k}_{1}$ and $\mathbf{k}_{2}$ whose sum is $\mathbf{k}$. It implies an integral of the form

$$
\mathcal{K}\left(\mathbf{k}_{1}, \mathbf{k}_{2}, \mathbf{k}\right) \equiv \int \frac{\mathrm{d}^{3} \mathbf{k}_{1} \mathrm{~d}^{3} \mathbf{k}_{2}}{(2 \pi)^{3 / 2}} \delta_{D}^{3}\left(\mathbf{k}_{1}+\mathbf{k}_{2}-\mathbf{k}\right),
$$

that we abbreviate in $\mathcal{K}$. We can only align $\mathbf{k}$ with the direction $\overline{\mathbf{e}}_{3}$, but not $\mathbf{k}_{1}$ or $\mathbf{k}_{2}$ at the same time. However for a first order quantity the components $X_{\ell}^{m}$ for a mode in a given direction $\hat{\mathbf{k}}_{1}$ can be obtained by rotating the components $X_{\ell}^{0}$ obtained when we had decided to align this direction with $\overline{\mathbf{e}}_{3}$. Namely this rotation leads for a mode $\mathbf{k}$ to

$$
X_{\ell}^{m}(\mathbf{k})=\sqrt{\frac{4 \pi}{2 \ell+1}} Y^{\star \ell m}(\hat{\mathbf{k}}) X_{\ell}^{0}\left(k \overline{\mathbf{e}}_{3}\right),
$$

and in particular for the first order velocity

$$
\hat{v}_{n}(\mathbf{k})=-\hat{k}^{(n)} \hat{v}_{0}\left(k \overline{\mathbf{e}}_{3}\right) .
$$

Note that the scalar product of two mode vectors is given by

$$
\mathbf{k}_{1} \cdot \mathbf{k}_{2}=k_{1}^{(0)} k_{2}^{(0)}-k_{1}^{(1)} k_{2}^{(-1)}-k_{1}^{(-1)} k_{2}^{(1)}=\sum_{n=-1}^{1}(-1)^{n} k_{1}^{(n)} k_{2}^{(-n)}
$$


7.6.1. The Liouville operator We finally obtain for the linear terms in the second order Liouville operator

$$
\begin{aligned}
& \mathcal{L}^{\#(2)}\left[\hat{\mathcal{I}}^{(2)}\right]_{\ell}^{m}(k)=\hat{\mathcal{I}}_{\ell}^{\prime m(2)}(k)+k\left[\frac{{ }_{0}^{0} \uparrow K_{\ell+1}^{m}}{2 \ell+3} \hat{\mathcal{I}}_{\ell+1}^{m(2)}(k)-\frac{{ }_{0}^{0} \downarrow K_{\ell}^{m}}{2 \ell-1} \hat{\mathcal{I}}_{\ell-1}^{m(2)}(k)\right] \\
& -\delta_{\ell}^{0} \delta_{m}^{0} 4 \Psi^{\prime(2)}(k)-\delta_{\ell}^{1} \delta_{m}^{0} 4 k \hat{\Phi}^{(2)}(k) \\
& +\delta_{\ell}^{2} \delta_{m}^{1} \frac{4 k}{\sqrt{3}} \hat{\Phi}_{1}^{(2)}(k)+\delta_{\ell}^{2} \delta_{m}^{-1} \frac{4 k}{\sqrt{3}} \hat{\Phi}_{-1}^{(2)}(k) \\
& +\delta_{\ell}^{2} \delta_{m}^{2} 4 \hat{H}_{1}^{\prime(2)}+\delta_{\ell}^{2} \delta_{m}^{-2} 4 \hat{H}_{-1}^{\prime(2)}, \\
& \mathcal{L}^{\#(2)}\left[\hat{\mathcal{E}}^{(2)}\right]_{\ell}^{m}(k)=\hat{\mathcal{E}}_{\ell}^{\prime m(2)}(k) \\
& +k\left[\frac{{ }_{2}^{0} \uparrow K_{\ell+1}^{m}}{2 \ell+3} \hat{\mathcal{E}}_{\ell+1}^{m(2)}(k)+\frac{2 m}{\ell(\ell+1)} \hat{\mathcal{B}}_{\ell}^{m(2)}(k)-\frac{{ }_{2}^{0} \downarrow K_{\ell}^{m}}{2 \ell-1} \hat{\mathcal{E}}_{\ell-1}^{m(2)}(k)\right], \\
& \mathcal{L}^{\#(2)}\left[\hat{\mathcal{B}}^{(2)}\right]_{\ell}^{m}(k)=\hat{\mathcal{B}}_{\ell}^{\prime m(2)}(k) \\
& +k\left[\frac{{ }_{2}^{0} \uparrow K_{\ell+1}^{m}}{2 \ell+3} \hat{\mathcal{B}}_{\ell+1}^{m(2)}(k)-\frac{2 m}{\ell(\ell+1)} \hat{\mathcal{E}}_{\ell}^{m(2)}(k)-\frac{{ }_{2}^{0} \downarrow K_{\ell}^{m}}{2 \ell-1} \hat{\mathcal{B}}_{\ell-1}^{m(2)}(k)\right] .
\end{aligned}
$$

As for the quadratic terms, we can use the composition rules given in Appendix D to obtain

$$
\begin{aligned}
& \mathcal{L}^{\#(1)(1)}[\hat{\mathcal{I}}]_{\ell}^{m}(k)=2 \mathcal{K}\left\{-\sum_{n=-1}^{1} \frac{{ }_{0}^{n} \uparrow K_{\ell+1}^{m}}{2 \ell+3} \hat{\mathcal{I}}_{\ell+1}^{m+n}\left(\mathbf{k}_{2}\right)\right. \\
& \times\left[-(\ell-2) k_{1}^{(-n)} \hat{\Phi}\left(k_{1}\right)+\left(k_{2}^{(-n)}-(\ell+2) k_{1}^{(-n)}\right) \hat{\Psi}\left(k_{1}\right)\right] \\
& +\sum_{n=-1}^{1} \frac{{ }_{n}^{n} \downarrow K_{\ell}^{m}}{2 \ell-1} \hat{\mathcal{I}}_{\ell-1}^{m-n}\left(\mathbf{k}_{2}\right) \\
& \times\left[(\ell+3) k_{1}^{(n)} \hat{\Phi}\left(k_{1}\right)+\left(k_{2}^{(n)}+(\ell-1) k_{1}^{(n)}\right) \hat{\Psi}\left(k_{1}\right)\right] \\
& -4 \hat{\Psi}^{\prime}\left(k_{1}\right) \hat{\mathcal{I}}_{\ell}^{m}\left(\mathbf{k}_{2}\right)-\hat{\Phi}\left(k_{1}\right) \hat{\mathcal{I}}_{\ell}^{\prime m}\left(\mathbf{k}_{2}\right) \\
& +4 \delta_{\ell}^{1}\left(\hat{\Psi}\left(k_{1}\right)-2 \hat{\Phi}\left(k_{1}\right)\right) \hat{\Phi}\left(k_{2}\right) k_{2}^{(m)} \\
& \left.+4 \delta_{\ell}^{0}\left[\hat{\Phi}\left(k_{1}\right)-2 \hat{\Psi}\left(k_{1}\right)\right] \hat{\Psi}^{\prime}\left(k_{2}\right)\right\}, \\
& \mathcal{L}^{\#(1)(1)}[\hat{\mathcal{E}}]_{\ell}^{m}(k)=2 \mathcal{K}\left\{-\sum_{n=-1}^{1} \frac{{ }^{n} \uparrow K_{\ell+1}^{m}}{2 \ell+3} \hat{\mathcal{E}}_{\ell+1}^{m+n}\left(\mathbf{k}_{2}\right)\right. \\
& \times\left[-(\ell-2) k_{1}^{(-n)} \hat{\Phi}\left(k_{1}\right)+\left(k_{2}^{(-n)}-(\ell+2) k_{1}^{(-n)}\right) \hat{\Psi}\left(k_{1}\right)\right] \\
& +\sum_{n=-1}^{1} \frac{2 \downarrow}{2 \ell-1} K_{\ell}^{m} \hat{\mathcal{E}}_{\ell-1}^{m-n}\left(\mathbf{k}_{2}\right) \\
& \times\left[(\ell+3) k_{1}^{(n)} \hat{\Phi}\left(k_{1}\right)+\left(k_{2}^{(n)}+(\ell-1) k_{1}^{(n)}\right) \hat{\Psi}\left(k_{1}\right)\right] \\
& \left.-4 \hat{\Psi}^{\prime}\left(k_{1}\right) \hat{\mathcal{E}}_{\ell}^{m}\left(\mathbf{k}_{2}\right)-\hat{\Phi}\left(k_{1}\right) \hat{\mathcal{E}}_{\ell}^{\prime m}\left(\mathbf{k}_{2}\right)\right\}
\end{aligned}
$$




$$
\begin{aligned}
\mathcal{L}^{\#(1)(1)}[\hat{\mathcal{B}}]_{\ell}^{m}(k)=2 \mathcal{K} \frac{2}{(\ell+1)}\{ & \sum_{n=-1}^{1}{ }^{n} \lambda_{\ell}^{m} \hat{\mathcal{E}}_{\ell}^{m-n}\left(\mathbf{k}_{2}\right) \times \\
& \left.\times\left[\left(k_{2}^{(n)}-k_{1}^{(n)}\right)\left(\hat{\Phi}\left(k_{1}\right)+\hat{\Psi}\left(k_{1}\right)\right)+4 k_{1}^{(n)} \hat{\Phi}\left(k_{1}\right)\right]\right\}
\end{aligned}
$$

This last expression and its version in PSTF multipoles (4.34), can be traced directly to the lensing term in the expression (4.30) and we recover the well known result that at second order, gravitational lensing generates magnetic type multipoles out of electric type multipoles.

7.6.2. The collision tensor Using a similar method applied to the collision term leads to

$\mathcal{C}^{\#(2)}[\hat{\mathcal{I}}]_{\ell}^{m}(k)=\bar{\tau}^{\prime}\left[-\hat{\mathcal{I}}_{\ell}^{m(2)}(k)+\delta_{\ell}^{0} \delta_{m}^{0} \hat{\mathcal{I}}_{0}^{0(2)}(k)+4 \delta_{\ell}^{1} \hat{v}_{m}^{(2)}(k)+\delta_{\ell}^{2} \hat{P}^{m(2)}(k)\right]$,

where $\hat{P}^{m(2)}(k)$ is not null only if $-2 \leq m \leq 2$ and is defined in that case by

$$
\hat{P}^{m(2)}(k)=\frac{1}{10}\left[\hat{\mathcal{I}}_{2}^{m(2)}(k)-\sqrt{6} \hat{\mathcal{E}}_{2}^{m(2)}(k)\right] .
$$

For the electric and magnetic type collision terms, their second order linear components read

$$
\begin{aligned}
& \mathcal{C}^{\#(2)}[\hat{\mathcal{E}}]_{\ell}^{m}(k)=\bar{\tau}^{\prime}\left[-\hat{\mathcal{E}}_{\ell}^{m}(k)-\delta_{\ell}^{2} \sqrt{6} \hat{P}^{m(2)}(k)\right], \\
& \mathcal{C}^{\#(2)}[\hat{\mathcal{B}}]_{\ell}^{m}(k)=-\bar{\tau}^{\prime} \hat{\mathcal{B}}_{\ell}^{m}(k) .
\end{aligned}
$$

The quadratic terms are then given by

$$
\begin{aligned}
\mathcal{C}^{\#(1)(1)}\left[\hat{\mathcal{I}}^{(1)}\right]_{\ell}^{m}(k)=2 \overline{\tau^{\prime} \mathcal{K}}\left\{-\sum_{n=-1}^{1} \frac{{ }_{0}^{n} \uparrow K_{\ell+1}^{m}}{2 \ell+3} \hat{\mathcal{I}}_{\ell+1}^{m+n}\left(\mathbf{k}_{2}\right) \hat{v}_{-n}\left(\mathbf{k}_{1}\right)\right. \\
+\sum_{n=-1}^{1} \frac{{ }_{0}^{n} \downarrow K_{\ell}^{m}}{2 \ell-1} \hat{\mathcal{I}}_{\ell-1}^{m-n}\left(\mathbf{k}_{2}\right) \hat{v}_{n}\left(\mathbf{k}_{1}\right) \\
+\delta_{\ell}^{0}\left[-\frac{4}{3} \sum_{n=-1}^{1}(-1)^{n} \hat{v}_{n}\left(\mathbf{k}_{1}\right) \hat{v}_{-n}\left(\mathbf{k}_{2}\right)\right. \\
\left.+\sum_{n=-1}{ }^{n} \frac{0 \uparrow K_{1}^{0}}{3} \hat{\mathcal{I}}_{1}^{n}\left(\mathbf{k}_{2}\right) \hat{v}_{-n}\left(\mathbf{k}_{1}\right)\right] \\
+\delta_{\ell}^{1} 3 \sum_{n=-1}^{1}{ }_{0}^{n} \downarrow K_{1}^{m} \hat{\mathcal{I}}_{0}^{m-n}\left(\mathbf{k}_{2}\right) \hat{v}_{n}\left(\mathbf{k}_{1}\right) \\
+\delta_{\ell}^{2} \sum_{n=-1}^{1} \frac{{ }^{n} \downarrow K_{2}^{m}}{3}\left[-\frac{1}{2} \hat{\mathcal{I}}_{1}^{m-n}\left(\mathbf{k}_{2}\right)+7 \hat{v}_{m-n}\left(\mathbf{k}_{2}\right)\right] \hat{v}_{n}\left(\mathbf{k}_{1}\right) \\
\left.+\delta_{\ell}^{3} \frac{1}{2} \sum_{n=-1}^{1} \frac{{ }_{0}^{n} \downarrow K_{3}^{m}}{5}\left[\hat{\mathcal{I}}_{2}^{m-n}\left(\mathbf{k}_{2}\right)-\sqrt{6} \hat{\mathcal{E}}_{2}^{m-n}\left(\mathbf{k}_{2}\right)\right] \hat{v}_{n}\left(\mathbf{k}_{1}\right)\right\} \\
+2 \hat{\mathcal{K}}^{(1)}\left(\mathbf{k}_{1}\right) \mathcal{C}^{\#(1)}\left[\hat{\mathcal{I}}^{(1)}\right]_{\ell}^{m}\left(\mathbf{k}_{2}\right),
\end{aligned}
$$




$$
\begin{aligned}
& \mathcal{C}^{\#(1)(1)}\left[\hat{\mathcal{E}}^{(1)}\right]_{\ell}^{m}(k)=2 \bar{\tau}^{\prime} \mathcal{K}\left\{-\sum_{n=-1}^{1} \frac{{ }^{n} \uparrow K_{\ell+1}^{m}}{2 \ell+3} \hat{\mathcal{E}}_{\ell+1}^{m+n}\left(\mathbf{k}_{2}\right) \hat{v}_{-n}\left(\mathbf{k}_{1}\right)\right. \\
& +\sum_{n=-1}^{1} \frac{{ }_{2}^{n} \downarrow K_{\ell}^{m}}{2 \ell-1} \hat{\mathcal{E}}_{\ell-1}^{m-n}\left(\mathbf{k}_{2}\right) \hat{v}_{n}\left(\mathbf{k}_{1}\right) \\
& +\delta_{\ell}^{2} \sum_{n=-1}^{1} \frac{{ }_{0}^{n} \downarrow K_{2}^{m}}{3}\left[\frac{\sqrt{6}}{2} \hat{\mathcal{I}}_{1}^{m-n}\left(\mathbf{k}_{2}\right)-\sqrt{6} \hat{v}_{m-n}\left(\mathbf{k}_{2}\right)\right] \hat{v}_{n}\left(\mathbf{k}_{1}\right) \\
& \left.+\delta_{\ell}^{3} \frac{1}{2} \sum_{n=-1}^{1} \frac{{ }_{2}^{n} \downarrow K_{3}^{m}}{5}\left[-\sqrt{6} \hat{\mathcal{I}}_{2}^{m-n}\left(\mathbf{k}_{2}\right)+6 \hat{\mathcal{E}}_{2}^{m-n}\left(\mathbf{k}_{2}\right)\right] \hat{v}_{n}\left(\mathbf{k}_{1}\right)\right\} \\
& +2 \mathcal{K}{\hat{\tau^{\prime}}}^{(1)}\left(\mathbf{k}_{1}\right) \mathcal{C}^{\#(1)}\left[\hat{\mathcal{E}}^{(1)}\right]_{\ell}^{m}\left(\mathbf{k}_{2}\right) \text {, } \\
& \mathcal{C}^{\#(1)(1)}\left[\hat{\mathcal{B}}^{(1)}\right]_{\ell}^{m}(k)=-2 \overline{\tau^{\prime} \mathcal{K}}\left\{\frac{-2}{(\ell+1)} \sum_{n=-1}^{1}{ }^{n} \lambda_{\ell}^{m} \hat{v}_{n}\left(\mathbf{k}_{1}\right) \hat{\mathcal{E}}_{\ell}^{m-n}\left(\mathbf{k}_{2}\right)\right. \\
& \left.-\delta_{\ell}^{2} \sum_{n=-1}^{1}{ }^{n} \lambda_{2}^{m} \hat{v}_{n}\left(\mathbf{k}_{1}\right)\left[\frac{4}{5} \hat{\mathcal{E}}_{2}^{m-n}\left(\mathbf{k}_{2}\right)-\frac{2}{15} \sqrt{6} \hat{\mathcal{I}}_{2}^{m-n}\left(\mathbf{k}_{2}\right)\right]\right\} .
\end{aligned}
$$

\subsection{Continuity and Euler equations for baryons}

As discussed in section 1.5 we describe the baryons by cold matter that is by a fluid with no pressure. From equations (5.33) (5.34) and (6.37) we obtain the continuity equations

$$
\begin{aligned}
& {\left[\frac{\hat{\rho}^{(1)}(k)}{\bar{\rho}}\right]^{\prime}+k \hat{v}_{0}^{(1)}(k)-3 \hat{\Psi}^{\prime(1)}(k)=0} \\
& {\left[\frac{\hat{\rho}^{(2)}(k)}{\bar{\rho}}\right]^{\prime}+k \hat{v}_{0}^{(2)}(k)-3 \hat{\Psi}^{\prime}(2)(k)} \\
& +2 \mathcal{K}\left\{-\sum_{n=-1}^{1}(-1)^{n}\left[\mathcal{H} \hat{v}_{n}\left(\mathbf{k}_{1}\right) \hat{v}_{-n}\left(\mathbf{k}_{2}\right)+\frac{\hat{\rho}\left(\mathbf{k}_{1}\right)}{\bar{\rho}} k_{1}^{(n)} \hat{v}_{-n}\left(\mathbf{k}_{2}\right)+2 \hat{v}_{n}\left(\mathbf{k}_{1}\right) \hat{v}_{-n}^{\prime}\left(\mathbf{k}_{2}\right)\right]\right. \\
& \quad+\left[\frac{\hat{\rho}\left(k_{1}\right)}{\bar{\rho}}+\hat{\Phi}\left(k_{1}\right)+\hat{\Psi}\left(k_{1}\right)\right] k_{2} \hat{v}_{0}\left(k_{2}\right)-3 \hat{\Psi}^{\prime}\left(k_{1}\right) \frac{\hat{\rho}\left(k_{2}\right)}{\bar{\rho}}-6 \hat{\Psi}\left(k_{1}\right) \hat{\Psi}^{\prime}\left(k_{2}\right) \\
& \left.\quad-2 \sum_{n=-1}^{1}(-1)^{n}\left[\hat{\Phi}\left(k_{2}\right)-\hat{\Psi}\left(k_{2}\right)\right] k_{2}^{(n)} \hat{v}_{-n}\left(\mathbf{k}_{1}\right)\right\} \\
& =-\frac{2 \bar{\tau}^{\prime}}{R} \mathcal{K} \sum_{n=-1}^{1}(-1)^{n}\left[\frac{1}{3} \hat{\mathcal{I}}_{1}^{n}\left(\mathbf{k}_{2}\right) \hat{v}_{-n}\left(\mathbf{k}_{1}\right)-\frac{4}{3} \hat{v}_{n}\left(\mathbf{k}_{1}\right) \hat{v}_{-n}\left(\mathbf{k}_{2}\right)\right]
\end{aligned}
$$

where $R \equiv \bar{\rho} / \overline{\mathcal{I}}$.

From equations (6.37) (5.35) and (5.36) we obtain the Euler equation for baryons

$$
\hat{v}_{m}^{\prime(1)}(k)+\mathcal{H} \hat{v}_{m}^{(1)}(k)-\delta_{m}^{0} k \hat{\Phi}^{(1)}(k)=-\frac{\bar{\tau}^{\prime}}{3 R}\left[-\hat{\mathcal{I}}_{1}^{m(1)}(k)+4 \hat{v}_{m}^{(1)}(k)\right],
$$




$$
\begin{aligned}
& \hat{v}_{m}^{\prime(2)}(k)+\mathcal{H} \hat{v}_{m}^{(2)}(k)-\delta_{m}^{0} k \hat{\Phi}^{(2)}(k) \\
& +2 \mathcal{K}\left\{\left[\frac{\hat{\rho}\left(k_{1}\right)}{\bar{\rho}}-\hat{\Phi}\left(k_{1}\right)\right]\left[\hat{v}_{m}^{\prime}\left(\mathbf{k}_{2}\right)+\mathcal{H} \hat{v}_{m}\left(\mathbf{k}_{2}\right)\right]+\left[\frac{\hat{\rho}\left(k_{1}\right)}{\bar{\rho}}+\hat{\Psi}\left(k_{1}\right)\right] k_{2}^{(m)} \hat{\Phi}\left(k_{2}\right)\right. \\
& \left.+\hat{v}_{m}\left(\mathbf{k}_{2}\right)\left[\left(\frac{\hat{\rho}\left(k_{1}\right)}{\bar{\rho}}\right)^{\prime}-4 \hat{\Psi}^{\prime}\left(k_{1}\right)\right]+\delta_{m}^{0} k \hat{\Phi}\left(k_{1}\right) \hat{\Phi}\left(k_{2}\right)-\hat{v}_{m}\left(\mathbf{k}_{2}\right) \sum_{n=-1}^{1}(-1)^{n} v_{n}\left(\mathbf{k}_{1}\right) k^{(-n)}\right\} \\
& =-\frac{\bar{\tau}^{\prime}}{3 R}\left[-\hat{\mathcal{I}}_{1}^{m(2)}(k)+4 \hat{v}_{m}^{(2)}(k)\right]-\frac{2}{3 R} \mathcal{K} \hat{\tau}^{(1)}\left(\mathbf{k}_{1}\right)\left[-\hat{\mathcal{I}}_{1}^{m(1)}\left(\mathbf{k}_{\mathbf{2}}\right)+4 \hat{v}_{m}^{(1)}\left(\mathbf{k}_{2}\right)\right] \\
& -\frac{2}{3 R} \bar{\tau}^{\prime} \mathcal{K}\left[4 \hat{\mathcal{I}}_{0}^{0}\left(\mathbf{k}_{2}\right) \hat{v}_{m}\left(\mathbf{k}_{1}\right)-\sum_{n=-1}^{1} \frac{{ }_{0}^{n} K_{2}^{m}}{5} \hat{\mathcal{I}}_{2}^{m+n}\left(\mathbf{k}_{2}\right) \hat{v}_{-n}\left(\mathbf{k}_{1}\right)\right] .
\end{aligned}
$$

\subsection{The road to numerical integration}

The set of equations which need to be integrated is very intricate. First the scalar $(m=0)$ first order equations need to be integrated for each Fourier mode magnitude $k$ (see [91, 92, 93]). This encompasses the first order Einstein equations to determine the first order scalar potentials, the baryons continuity equation (7.55) and Euler equation (7.56), together with the Boltzmann infinite hierarchy of coupled equations for the intensity and electric type multipoles given by the first order Liouville operator multipoles (7.34) and (7.35) equated with the first order collision multipoles (7.36) and (7.37). The first order Boltzmann hierarchy couples the modes $\ell$ with modes $\ell \pm 1$. The results obtained depend only on the magnitude of a given mode since we chose to align the mode with the azimuthal direction of the spherical harmonics. The multipoles for any direction of the Fourier mode is obtained by the rotation (7.39). Finally, we obtain from this first order numerical integration a first order transfer function $\mathcal{T}_{\ell}^{m(1)}(\mathbf{k}, \eta)$ defined by

$$
\hat{\mathcal{I}}_{\ell}^{m(1)}(\mathbf{k}, \eta)=\mathcal{T}_{\ell}^{m(1)}(\mathbf{k}, \eta) \overline{\mathcal{I}}_{\ell}^{m}(\eta) \zeta(k),
$$

where $\zeta(k)$ is the primordial curvature perturbation in comoving gauge.

At second order the numerical integration has to be performed in the same manner. We have to integrate simultaneously the second order Einstein equations in order to obtain the metric perturbations $\hat{\Phi}^{(2)}, \hat{\Psi}^{(2)}, \hat{\Phi}_{I}^{(2)}$ and $\hat{H}_{I J}^{(2)}$ (the expressions of the second order Einstein tensor can be found for instance in [43, 94, 95, 96]), the second order continuity equation for baryons (7.56) and the second order Euler equation (7.57), together with the second order Boltzmann infinite hierarchy for intensity, electric type, and magnetic type multipoles which is obtained from the second order Liouville operator multipoles (7.42 7.47 ) equated with the second order collision multipoles (7.48 $[7.54)$. After numerical integration we will obtain a second order transfer function $\mathcal{T}_{\ell}^{m(2)}\left(\mathbf{k}_{1}, \mathbf{k}_{2}\right)$ defined by

$$
\frac{1}{2} \hat{\mathcal{I}}_{\ell}^{m(2)}(k, \eta)=\mathcal{K} \mathcal{T}_{\ell}^{m(2)}\left(\mathbf{k}_{1}, \mathbf{k}_{2}, \eta\right) \overline{\mathcal{I}}_{\ell}^{m}(\eta) \zeta\left(k_{1}\right) \zeta\left(k_{2}\right) .
$$

However, we already see that the Boltzmann hierarchy at second order is much more complex than its first order counterpart. First it depends on the two modes $\mathbf{k}_{1}$ and $\mathbf{k}_{2}$ (though their sum $\mathbf{k}$ is chosen to be aligned with the azimuthal direction of the spherical harmonics) inside the convolution in quadratic terms. Second, it couples a 
mode $(\ell, m)$ with the modes $(\ell \pm 1, m \pm 1)$. And finally, one problem which needs to be solved is the determination of $\tau^{\prime(1)}$. Indeed, photons can only scatter off free electrons and its correct expression is $\tau^{\prime}=a n_{\mathrm{e}} x_{\mathrm{e}} \sigma_{\mathrm{T}}$, where $x_{\mathrm{e}}$ is the fraction of ionized electrons. Both $\hat{n}_{\mathrm{e}}^{(1)}$ and $\hat{x}_{\mathrm{e}}^{(1)}$ contribute to the first order perturbation since $\hat{\tau}^{\prime(1)}=a \sigma_{\mathrm{T}} \hat{x}_{\mathrm{e}}^{(1)} \bar{n}_{\mathrm{e}}+a \sigma_{\mathrm{T}} \bar{x}_{\mathrm{e}} \hat{n}_{\mathrm{e}}^{(1)}$. Though $\hat{n}_{\mathrm{e}}^{(1)}$ is easily obtained from the first order equations using $\hat{n}_{\mathrm{e}}^{(1)} / \bar{n}_{\mathrm{e}}=\hat{\rho}_{\mathrm{e}}^{(1)} / \bar{\rho}_{\mathrm{e}}$, the perturbation of $x_{\mathrm{e}}$ requires to determine and integrate the equations which rule the physics of recombination on the first order perturbed space-time.

\section{Conclusion}

In this paper we have extended our previous investigation [45] to the case of polarized radiation, also including the Compton scattering of photons off free electrons, in order to obtain a fully consistent second order treatment of the radiation transfer. We also studied the case of massive particles in the kinetic theory and checked that in the case $T / m \ll 1$ it was consistent with a fluid approximation up to second order. Our analysis is based on the careful definition of a locally Minkowski frame which thanks to the equivalence principle simplifies the treatment of local interactions. Though this method was already implicitly followed in the existing literature, we have performed a deep analysis of its implications for the gauge transformation, the SVT decomposition and the evolution equations. At first order it provides a more satisfactory understanding of the formalism used in the literature, whereas at second order it appears that it is completely necessary to master the formal aspects that we have presented in this paper in order to understand the physical meaning of the dynamical equations. For this purpose we have introduced a font and color based notation which should clarify the formalism. The results that we have presented should now be the starting point to a numerical integration of the second order radiative transfer, but it appears already to be a huge and long term task if we want it to be computationally efficient. Similarly to the first order, approximate schemes should be first developed in order to obtain the main features, and this first step has already been taken in 44 for small angular scales.

\section{Acknowledgments}

I thank J. Martin-Garcia for his help in using the tensorial calculus packages xPert 97. and xAct 71 that were used to derive the second order expressions of this paper and to calculate the integrals in equation (1.49). I thank G. Faye for his help on the equation (7.29). I thank Jean-Philippe Uzan for having guided me into the realm of $\mathrm{CMB}$, and IAP where most of this work was carried out.

\section{Appendix A. Transformation rules for multipoles at second order in $\mathbf{v}$}

Following the method of section 1.7 to compute the transformation rules of the radiation multipoles under a change of frame, we find the following rules up to second order in $\mathbf{v}$ for the multipoles used in section 6.5 (note that contrary to the choice we

made in section 1.7.2 we express the result in function of the $I_{\underline{a_{\ell}}}^{\{n\}}\left(p^{o}\right)$ and not the 
$I_{\underline{a_{\ell}}}^{\{n\}}\left(p^{\tilde{o}}\right)$ since this is what was required to transform the collision tensor in section 6.5)

$$
\begin{aligned}
& \tilde{I}_{\emptyset}\left(p^{\tilde{o}}\right)= I_{\emptyset} \\
&\left(p^{o}\right)-I_{\emptyset}^{\prime}\left(p^{o}\right) \mathbf{n} \cdot \mathbf{v}+I_{\emptyset}^{\prime}\left(p^{o}\right) \mathbf{v} \cdot \mathbf{v}+\frac{1}{6} I_{\emptyset}^{\prime \prime}\left(p^{o}\right) \mathbf{v} \cdot \mathbf{v}+\frac{1}{2} I_{\emptyset}^{\prime \prime}\left(p^{o}\right)(\mathbf{n} \cdot \mathbf{v})^{2} \\
&+\frac{2}{3} v^{i} I_{i}\left(p^{o}\right)+\frac{1}{3} v^{i} I_{i}^{\prime}\left(p^{o}\right)-\frac{1}{3} I_{i}^{\prime \prime}\left(p^{o}\right) v^{i} \mathbf{v} \cdot \mathbf{n}-I_{i}^{\prime}\left(p^{o}\right) v^{i} \mathbf{v} \cdot \mathbf{n} \\
&+\frac{2}{5} I_{i j}\left(p^{o}\right) v^{i} v^{j}+\frac{2}{5} I_{i j}^{\prime}\left(p^{o}\right) v^{i} v^{j}+\frac{1}{15} I_{i j}^{\prime \prime}\left(p^{o}\right) v^{i} v^{j} \\
& \tilde{I}_{\tilde{\imath} \tilde{\jmath}}\left(p^{\tilde{o}}\right)= I_{i j}\left(p^{o}\right)+\frac{1}{2} I_{\emptyset}^{\prime \prime}\left(p^{o}\right) v_{\langle i} v_{j\rangle}-I_{\langle i}\left(p^{o}\right) v_{j\rangle}+I_{\langle i}^{\prime}\left(p^{o}\right) v_{j\rangle}-I_{\langle i}^{\prime \prime}\left(p^{o}\right) v_{j\rangle} \mathbf{n} \cdot \mathbf{v} \\
&-I_{i j}^{\prime}\left(p^{o}\right) \mathbf{n} \cdot \mathbf{v}-\frac{4}{7} I_{i j}\left(p^{o}\right) \mathbf{v} \cdot \mathbf{v}+\frac{5}{7} I_{i j}^{\prime}\left(p^{o}\right) \mathbf{v} \cdot \mathbf{v}+\frac{1}{14} I_{i j}^{\prime \prime}\left(p^{o}\right) \mathbf{v} \cdot \mathbf{v}+\frac{1}{2} I_{i j}^{\prime \prime}\left(p^{o}\right)(\mathbf{n} \cdot \mathbf{v})^{2} \\
&-\frac{9}{7} I_{k\langle i}\left(p^{o}\right) v_{j\rangle} v^{k}+\frac{6}{7} I_{k\langle i}^{\prime}\left(p^{o}\right) v_{j\rangle} v^{k}+\frac{2}{7} I_{k\langle i}^{\prime \prime}\left(p^{o}\right) v_{j\rangle} v^{k} \\
&+\frac{12}{7} I_{i j k}\left(p^{o}\right) v^{k}+\frac{3}{7} I_{i j k}^{\prime}\left(p^{o}\right) v^{k}-\frac{15}{7} I_{i j k}^{\prime}\left(p^{o}\right) v^{k} \mathbf{n} \cdot \mathbf{v}-\frac{3}{7} I_{i j k}^{\prime \prime}\left(p^{o}\right) v^{k} \mathbf{n} \cdot \mathbf{v} \\
&+\frac{40}{21} I_{i j k l}\left(p^{o}\right) v^{k} v^{l}+\frac{20}{21} I_{i j k l}^{\prime}\left(p^{o}\right) v^{k} v^{l}+\frac{2}{21} I_{i j k l}^{\prime \prime}\left(p^{o}\right) v^{k} v^{l} \\
& \tilde{E}_{\tilde{\imath} \tilde{\jmath}}\left(p^{\tilde{o}}\right)= E_{i j}\left(p^{o}\right)-\frac{2}{21} E_{i j}\left(p^{o}\right) \mathbf{v} \cdot \mathbf{v}+\frac{11}{14} E_{i j}^{\prime}\left(p^{o}\right) \mathbf{v} \cdot \mathbf{v}+\frac{11}{42} E_{i j}^{\prime \prime}\left(p^{o}\right) \mathbf{v} \cdot \mathbf{v} \\
&+\frac{5}{7} E_{k\langle i}\left(p^{o}\right) v_{j\rangle} v^{k}-\frac{6}{7} E_{k\langle i}^{\prime}\left(p^{o}\right) v_{j\rangle} v^{k}-\frac{2}{7} E_{k\langle i}^{\prime \prime}\left(p^{o}\right) v_{j\rangle} v^{k} \\
&+\frac{20}{21} E_{i j k}\left(p^{o}\right) v_{k}+\frac{5}{21} E_{i j k}^{\prime}\left(p^{o}\right) v_{k} \\
&+\frac{50}{63} E_{i j k l}\left(p^{o}\right) v^{k} v^{l}+\frac{25}{63} E_{i j k l}^{\prime}\left(p^{o}\right) v^{k} v^{l}+\frac{5}{126} E_{i j k l}^{\prime \prime}\left(p^{o}\right) v^{k} v^{l} \\
&+\frac{1}{3} v_{k} \epsilon_{\langle i}^{k l} B_{j\rangle l}\left(p^{o}\right)+\frac{1}{3} v_{k} \epsilon^{k l}{ }_{i i} B_{j\rangle l}^{\prime}\left(p^{o}\right) \\
&-\frac{10}{21} \epsilon_{\langle i}^{l m} B_{j\rangle k m}\left(p^{o}\right) v^{k} v_{l}-\frac{5}{7} \epsilon^{l m}{ }_{\langle i} B_{j\rangle k m}^{\prime}\left(p^{o}\right) v^{k} v_{l}-\frac{5}{42} \epsilon_{\langle i}^{l m} B_{j\rangle k m}^{\prime \prime}\left(p^{o}\right) v^{k} v_{l} .
\end{aligned}
$$

As for their energy integrated counterparts, they transform as

$$
\begin{aligned}
& \tilde{\mathcal{I}}_{\emptyset}=\mathcal{I}_{\emptyset}+\frac{4}{3} \mathcal{I}_{\emptyset \mathbf{V} . \mathbf{v}}-\frac{2}{3} \mathcal{I}_{i} v^{i}+\frac{2}{15} \mathcal{I}_{i j} v^{i} v^{j} \\
& \tilde{\mathcal{I}}_{i j}=\mathcal{I}_{i j}+10 \mathcal{I}_{\emptyset} v_{\langle i} v_{j\rangle}-5 \mathcal{I}_{\langle i} v_{j\rangle}+\mathcal{I}_{k\langle i} v_{j\rangle} v^{k} \\
& \tilde{\mathcal{E}}_{i j}=\mathcal{E}_{i j}-3 \mathcal{E}_{k\langle i} v_{j\rangle} v^{k}+2 \mathcal{E}_{i j} \mathbf{v} \cdot \mathbf{v}+2 v_{k} \epsilon^{k l}{ }_{\langle i} \mathcal{B}_{j\rangle l} .
\end{aligned}
$$




\section{Appendix B. Sources terms in second order transformations}

The perturbation variables in the decomposition (2.1) are extracted as follows

$$
\begin{aligned}
\Phi^{(n)} & =-\frac{1}{2 a^{2}} g_{O O}^{(n)}, \\
\Psi^{(n)} & =-\frac{1}{4 a^{2}} \mathrm{P}_{v}^{I J} g_{I J}^{(n)} \\
B^{(n)} & =\frac{1}{a^{2}} \mathrm{P}_{s}^{I} g_{O I}^{(n)} \\
B_{I}^{(n)} & =\frac{1}{a^{2}} \mathrm{P}_{v I}^{J} g_{O J}^{(n)}, \\
E^{(n)} & =\frac{1}{4 a^{2}}(\Delta \Delta)^{-1}\left(3 \partial^{I} \partial^{J}-\Delta \delta^{I J}\right) g_{I J}^{(n)}, \\
E_{N}^{(n)} & =\frac{1}{a^{2}} \mathrm{P}_{v N}{ }_{N} \mathrm{P}_{s}^{K}\left(\delta_{K}^{I} \delta_{L}^{J}-\frac{1}{3} \delta_{K L} \delta^{I J}\right) g_{I J}^{(n)} \\
H_{M N}^{(n)} & =\frac{1}{2 a^{2}} \mathrm{P}_{v M}^{K} \mathrm{P}_{v N}^{L}\left(\delta_{K}^{I} \delta_{L}^{J}-\frac{1}{3} \delta_{K L} \delta^{I J}\right) g_{I J}^{(n)},
\end{aligned}
$$

where $n=1,2$ is the order and where we have used the definitions

$$
\mathrm{P}_{s}^{I} \equiv \Delta^{-1} \partial^{I}, \quad \mathrm{P}_{v}^{I J} \equiv \delta^{I J}-\Delta^{-1} \partial^{I} \partial^{J} .
$$

Using this method we can read the source terms defined in equation (2.31), which are quadratic in the gauge change variables $T, L$ and the perturbation variables $\Phi, \Psi, B, E, E_{I J}$

$$
\begin{aligned}
S_{\Phi}= & \left(T^{\prime \prime}+5 \mathcal{H} T^{\prime}+\left(\mathcal{H}^{\prime}+2 \mathcal{H}^{2}\right) T+4 \mathcal{H} \Phi+2 \Phi^{\prime}\right)+\partial_{I} L^{\prime} \partial^{I}\left(T-2 B-L^{\prime}\right) \\
& +T^{\prime}\left(2 T^{\prime}+4 \Phi\right)+\partial_{I} L \partial^{I}\left(T^{\prime}+\mathcal{H} T+2 \Phi\right) \\
S_{\Psi}= & -T\left(\mathcal{H} T^{\prime}+\left(\mathcal{H}^{\prime}+2 \mathcal{H}^{2}\right) T-2 \Psi^{\prime}-4 \mathcal{H} \Psi\right)-\partial_{I}(\mathcal{H} T-2 \Psi) \partial^{I} L \\
& -\frac{1}{2}\left(\delta^{I J}-\Delta^{-1} \partial^{I} \partial^{J}\right) \mathcal{X}_{I J} \\
S_{B}= & \mathrm{P}_{s}{ }^{I} \mathcal{X}_{I} \\
S_{B_{I}}= & \mathrm{P}_{v I}{ }_{I}^{J} \mathcal{X}_{J} \\
S_{E}= & (\Delta \Delta)^{-1}\left(\frac{3}{2} \partial^{I} \partial^{J}-\frac{1}{2} \Delta \delta^{I J}\right) \mathcal{X}_{I J} \\
S_{E_{N}}= & 2 \mathrm{P}_{v N}{ }_{v}^{L} \mathrm{P}_{s}{ }^{K}\left(\delta_{K}^{I} \delta_{L}^{J}-\frac{1}{3} \delta_{K L} \delta^{I J}\right) \mathcal{X}_{I J} \\
S_{H_{M N}}= & \mathrm{P}_{v N}{ }_{N}^{L} \mathrm{P}_{v M}{ }_{v}^{K}\left(\delta_{K}^{I} \delta_{L}^{J}-\frac{1}{3} \delta_{K L} \delta^{I J}\right) \mathcal{X}_{I J},
\end{aligned}
$$

with

$$
\begin{aligned}
\mathcal{X}_{I} \equiv\{ & T^{\prime} \partial_{I}\left(2 B+L^{\prime}-T\right)+2 \mathcal{H} T \partial_{I}\left(2 B+L^{\prime}-T\right) \\
& +\partial^{J} L^{\prime}\left[2 \partial_{I} \partial_{J} L+2(\mathcal{H} T-2 \Psi) \delta_{I J}+4\left(H_{I J}+\partial_{I} \partial_{J} E\right)\right] \\
& +\partial^{J} \partial_{I} L \partial_{J}\left(2 B+L^{\prime}-T\right)+\partial^{J} L \partial_{J} \partial_{I}\left(2 B+L^{\prime}-T\right) \\
& \left.+\partial_{I} T\left(-4 \Phi-2 T^{\prime}-2 \mathcal{H} T\right)+T \partial_{I}\left(2 B^{\prime}+L^{\prime \prime}-T^{\prime}\right)\right\}
\end{aligned}
$$




$$
\begin{aligned}
\mathcal{X}_{I J} \equiv\left\{\partial_{J}\right. & \left(2 B+L^{\prime}-T\right) \partial_{I} T+T \partial_{I} \partial_{J}\left(L^{\prime}+2 \mathcal{H} L\right) \\
& +\partial_{I} \partial^{K} L\left[2 \partial_{K} \partial_{J} L+4 \partial_{K} \partial_{J} E+4 H_{K J}+(2 \mathcal{H} T-4 \Psi) \delta_{K J}\right] \\
& +T\left(2 H_{I J}^{\prime}+2 \partial_{I} \partial_{J} E^{\prime}+4 \mathcal{H} H_{I J}+4 \mathcal{H} \partial_{I} \partial_{J} E\right) \\
& \left.+\partial^{K} L \partial_{K}\left(\partial_{I} \partial_{J} L+2 H_{I J}+2 \partial_{I} \partial_{J} E\right)\right\} .
\end{aligned}
$$

As for the matter perturbation variables, the source terms in the transformation rules are

$$
\begin{aligned}
& S_{\rho}=T\left(\bar{\rho}^{\prime \prime} T+\bar{\rho}^{\prime} T^{\prime}+2 \delta \rho^{\prime}\right)+\partial^{I} L \partial_{I}\left(2 \delta \rho+\bar{\rho}^{\prime} T\right) \\
& S_{P}=T\left(\bar{P}^{\prime \prime} T+\bar{P}^{\prime} T^{\prime}+2 \delta P^{\prime}\right)+\partial^{I} L \partial_{I}\left(2 \delta P+\bar{P}^{\prime} T\right) \\
& S_{V}=\mathrm{P}_{s I}\left[\mathcal{H} T \partial^{I}\left(L^{\prime}-2 V\right)+T \partial^{I}\left(2 V^{\prime}-L^{\prime \prime}\right)+\partial^{J}\left(L^{\prime}-2 V\right) \partial_{J} \partial^{I} L\right. \\
& \left.+L^{J} \partial_{J} \partial^{I}\left(2 V-L^{\prime}\right)+\partial^{I} L^{\prime}\left(\mathcal{H} T+T^{\prime}+2 \Phi\right)\right] \\
& S_{\tilde{V}^{K}}=\mathrm{P}_{v}^{K}\left[\mathcal{H} T \partial^{I}\left(L^{\prime}-2 V\right)+T \partial^{I}\left(2 V^{\prime}-L^{\prime \prime}\right)+\partial^{J}\left(L^{\prime}-2 V\right) \partial_{J} \partial^{I} L\right. \\
& \left.+L^{J} \partial_{J} \partial^{I}\left(2 V-L^{\prime}\right)+\partial^{I} L^{\prime}\left(\mathcal{H} T+T^{\prime}+2 \Phi\right)\right] \\
& S_{\pi^{I J}}=2 T\left(\pi^{I J}\right)^{\prime}+2 \partial^{K} L \partial_{K} \pi^{I J}-2 \pi^{I K} \partial_{K} \partial^{J} L-2 \pi^{J K} \partial_{K} \partial^{I} L .
\end{aligned}
$$

\section{Appendix C. Perturbation of tetrads and Ricci rotation coefficients}

Appendix C.1. The perturbation of tetrads

At first order the coefficients of $R_{a b}$ and $S_{a b}$ are (remembering that we discard the first order vector modes)

$$
\begin{aligned}
& { }_{X} R_{o o}^{(1)}=-{ }_{X} S_{o o}^{(1)}=\Phi^{(1)} \\
& { }_{X} R_{o i}^{(1)}=-{ }_{X} S_{o i}^{(1)}=-\partial_{I} B^{(1)} \\
& { }_{X} R_{i o}^{(1)}=-{ }_{X} S_{i o}^{(1)}=0 \\
& { }_{X} R_{i k}^{(1)}=-S_{i k}^{(1)}=\Psi^{(1)} \delta_{I K}-\partial_{K} \partial_{I} E^{(1)}-H_{I K}^{(1)}
\end{aligned}
$$

We can read directly from these expressions the transformation rules for the tetrad when going from a gauge $X$ to a gauge $Y$

$$
\begin{aligned}
{ }_{Y} \mathbf{e}_{o}^{(1)} & =\mathcal{T}\left({ }_{X} \mathbf{e}_{o}^{(1)}\right)=-\mathcal{T}\left(\Phi^{(1)}\right) \overline{\mathbf{e}}_{o}-\overline{\mathbf{e}}_{i} \partial^{I} \mathcal{T}\left(B^{(1)}\right) \\
{ }_{Y} \mathbf{e}_{i}^{(1)} & =\mathcal{T}\left({ }_{X} \mathbf{e}_{i}^{(1)}\right)=\mathcal{T}\left(\Psi^{(1)}\right) \overline{\mathbf{e}}_{i}-\overline{\mathbf{e}}_{k} \partial^{K} \partial_{I} \mathcal{T}\left(E^{(1)}\right) .
\end{aligned}
$$

At second order the coefficients of $R_{a b}$ and $S_{a b}$ are

$$
\begin{aligned}
{ }_{X} R_{o o}^{(2)}= & \Phi^{(2)}-3 \Phi^{2}+\partial_{I} B \partial^{I} B \\
{ }_{X} R_{o i}^{(2)}= & -\partial_{I} B^{(2)}-B_{I}^{(2)}+(2 \Phi-4 \Psi) \partial_{I} B+4 \partial^{J} B\left(\partial_{I} \partial_{J} E+H_{I J}\right) \\
{ }_{X} R_{i o}^{(2)}= & -{ }_{X} S_{i o}^{(2)}=0 \\
{ }_{X} R_{i k}^{(2)}= & -{ }_{X} S_{i k}^{(2)} \\
= & \Psi^{(2)} \delta_{I K}-\left(\partial_{K} \partial_{I} E^{(2)}+\partial_{(K} E_{I)}^{(2)}+H_{K I}^{(2)}\right)+3 \Psi^{2} \delta_{I K} \\
& +3\left(\partial_{I} \partial^{L} E+H_{I}^{L}\right)\left(\partial_{L} \partial_{K} E+H_{L K}\right)-6 \Psi\left(\partial_{I} \partial_{K} E+H_{I K}\right) \\
{ }_{X} S_{o o}^{(2)}= & \Phi^{(2)}-\Phi^{2}+\partial_{I} B \partial^{I} B \\
{ }_{X} S_{o i}^{(2)}= & -\partial_{I} B^{(2)}-2 \Psi \partial_{I} B+2 \partial^{J} B\left(\partial_{I} \partial_{J} E+H_{I J}\right)
\end{aligned}
$$


The transformations rules for the tetrads can then be read

$$
\begin{aligned}
\mathcal{T}\left({ }_{X} \mathbf{e}_{o}^{(2)}\right)=- & {\left[\mathcal{T}\left(\Phi^{(2)}\right)-3 \mathcal{T}(\Phi)^{2}+\partial_{I} \mathcal{T}(B) \partial^{I} \mathcal{T}(B)\right] \overline{\mathbf{e}}_{o} } \\
& +\left\{-\partial^{I} \mathcal{T}\left(B^{(2)}\right)-\mathcal{T}\left(B^{I(2)}\right)+[2 \mathcal{T}(\Phi)-4 \mathcal{T}(\Psi)] \partial^{I} \mathcal{T}(B)\right. \\
& \left.+4 \partial^{J} \mathcal{T}(B)\left[\partial^{I} \partial_{J} \mathcal{T}(E)+H_{J}^{I}\right]\right\} \overline{\mathbf{e}}_{i} \\
\mathcal{T}\left({ }_{X} \mathbf{e}_{i}^{(2)}\right)=[ & \left.\mathcal{T}\left(\Psi^{(2)}\right)+3 \mathcal{T}(\Psi)^{2}\right] \overline{\mathbf{e}}_{i} \\
& +\left\{-\partial^{K} \partial_{I} \mathcal{T}\left(E^{(2)}\right)-\partial^{(K} \mathcal{T}\left(E_{I)}^{(2)}\right)-6 \mathcal{T}(\Psi)\left[\partial^{K} \partial_{I} \mathcal{T}(E)+H_{I}^{K}\right]\right. \\
& \left.+3\left[\partial_{I} \partial^{J} \mathcal{T}(E)+H_{I}^{J}\right]\left[\partial^{K} \partial_{J} \mathcal{T}(E)+H_{J}^{K}\right]\right\} \overline{\mathbf{e}}_{k} .
\end{aligned}
$$

Appendix C.2. The perturbation of Ricci rotation coefficients

If we use a tetrad basis, then the covariant derivative is characterized by the Ricci rotation coefficients $[72$ defined by

$$
\omega_{a b c} \equiv \eta_{b d} e_{\nu}^{d} e_{a}^{\mu} \nabla_{\mu} e_{c}^{\nu}=-\omega_{a c b} .
$$

They are related to the Christoffel symbols according to

$$
\omega_{a}{ }^{b}{ }_{c}=\Gamma_{A C}^{B} e_{a}^{C} e_{c}^{A} e_{B}^{b}+e_{a}^{C} e_{B}^{b} \partial_{C} e_{c}^{B} .
$$

We choose a background tetrad field which is adapted to the Cartesian coordinate system (see section 3.3). We then expand the Ricci rotation coefficients by expanding both the Christoffel symbols and the tetrads. Since the Ricci rotation coefficients are antisymmetric in their two last indices, $\omega_{\text {ooo }}=\omega_{i o o}=0$ up to any order. For the background metric the only non-vanishing components are

$$
\bar{\omega}_{i o j}=-\bar{\omega}_{i j o}=-\frac{\mathcal{H}}{a} \delta_{i j} .
$$

We can check that at this order the vectors in the tetrad commute (since they arise from a coordinate system) as

$$
0=\bar{\omega}_{a b c}-\bar{\omega}_{c b a}=\eta_{d b} \bar{e}_{\nu}^{d}\left[\bar{e}_{a}, \bar{e}_{c}\right]^{\nu} .
$$

At first order, restricting to the Newtonian gauge and neglecting the vector perturbations, the non-vanishing components are

$$
\begin{aligned}
\omega_{o o i}^{(1)} & =-\omega_{o i o}^{(1)}=-\frac{1}{a} \partial_{I} \Phi \\
\omega_{i o j}^{(1)} & =-\omega_{i j o}^{(1)}=\frac{1}{a}\left[-H_{I J}^{\prime}+\left(\mathcal{H} \Phi+\Psi^{\prime}\right) \delta_{I J}\right] \\
\omega_{j i k}^{(1)} & =-\omega_{j k i}^{(1)}=\frac{2}{a}\left(\partial_{[K} H_{I] J}-\partial_{[K} \Psi \delta_{I] J}\right) \\
\omega_{o i j}^{(1)} & =0 .
\end{aligned}
$$

As for the second order, the components that we have used in this paper are in the Newtonian gauge

$$
\begin{aligned}
\omega_{o o i}^{(2)}=-\omega_{\text {oio }}^{(2)}=\frac{1}{a} & {\left[-\partial_{I} \Phi^{(2)}+4 \Phi \partial_{I} \Phi+2 H_{I K} \partial^{K} \Phi-2 \Psi \partial_{I} \Phi\right] } \\
\omega_{i o j}^{(2)}=-\omega_{i j o}^{(2)}=\frac{1}{a} & \left\{\partial_{(I} B_{J)}^{(2)}-H_{I J}^{(2))^{\prime}}+\left[\mathcal{H} \Phi^{(2)}+\Psi^{(2)^{\prime}}\right] \delta_{I J}-3 \mathcal{H} \Phi^{2} \delta_{I J}\right. \\
& +2\left(H_{I J}^{\prime}-\Psi^{\prime} \delta_{I J}\right) \Phi+2\left[H_{I K}^{\prime}-\Psi^{\prime} \delta_{I K}\right]\left[H_{J}^{K}-\Psi \delta_{J}^{K}\right] \\
& \left.+2\left[H_{J K}^{\prime}-\Psi^{\prime} \delta_{J K}\right]\left[H_{I}^{K}-\Psi \delta_{I}^{K}\right]\right\} .
\end{aligned}
$$


It can be checked that the vectors in the tetrad do not commute at the perturbed level as $\omega_{a b c}^{(1)} \neq \omega_{c b a}^{(1)}$ and $\omega_{a b c}^{(2)} \neq \omega_{c b a}^{(2)}$.

\section{Appendix D. Useful formulas for extracting the normal modes}

From the general composition rule (7.29), we obtain the following useful particular cases which can be used to extract the normal modes on quadratic terms

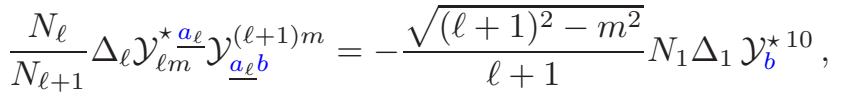

$$
\begin{aligned}
& \frac{N_{\ell}}{N_{\ell+1}} \Delta_{\ell} \mathcal{Y}_{\ell m}^{\star} \underline{a}_{\underline{\ell}} \mathcal{Y}_{\underline{a_{\ell}} b}^{(\ell+1)(m \pm 1)}=\frac{\sqrt{(\ell+1 \pm m)(\ell+2 \pm m)}}{\sqrt{2}(\ell+1)} N_{1} \Delta_{1} \mathcal{Y}_{b}^{\star 1(\mp 1)} \\
& \frac{N_{\ell}}{N_{\ell-1}} \Delta_{\ell} \mathcal{Y}_{\ell m}^{\star a_{\ell-1} b} \mathcal{Y}_{\underline{a_{\ell-1}}}^{(\ell-1) m}=\frac{\sqrt{\ell^{2}-m^{2}}}{(2 \ell-1)} N_{1} \Delta_{1} \mathcal{Y}_{b}^{\star 10}, \\
& \frac{N_{\ell}}{N_{\ell-1}} \Delta_{\ell} \mathcal{Y}_{\ell m}^{\star a_{\ell-1} b} \mathcal{Y}_{\underline{a_{\ell-1}}}^{(\ell-1)(m \pm 1)}=\frac{\sqrt{(\ell \mp m)(\ell \mp m-1)}}{\sqrt{2}(2 \ell-1)} N_{1} \Delta_{1} \mathcal{Y}_{b}^{\star 1(\mp 1)} \text {, }
\end{aligned}
$$

In order to extract normal modes in quadratic terms involving a curl, we also need the following useful formulas

$$
\begin{aligned}
& \mathrm{ie}_{b}^{(0)} \epsilon_{\left\langle a_{1}\right.}^{b c} \mathcal{Y}_{\left.\underline{a_{(\ell-1)}}\right\rangle c}^{\ell m}=-\frac{m}{\ell} \mathcal{Y}_{\underline{a_{\ell}}}^{\ell m}, \\
& \mathrm{i}_{b}^{( \pm 1)} \epsilon_{\left\langle a_{1}\right.}^{b c} \mathcal{Y}_{\left.\underline{a_{(\ell-1)}}\right\rangle c}^{\ell(m \mp 1)}= \pm \frac{1}{\ell} \sqrt{\frac{(\ell \pm m)(\ell+1 \mp m)}{2}} \mathcal{Y}_{\underline{a_{\ell}}}^{\ell m} .
\end{aligned}
$$

\section{References}

[1] J. Ehlers, General Relativity and Kinetic theory (Academic Press, New-York, USA, 1971).

[2] J. Bernstein, Kinetic theory in the expanding universe (Univ. Pr. 149p, Cambridge, USA, 1988).

[3] J. Stewart, Non-equilibrium relativistic kinetic theory, Lecture Notes in Physics Vol. 10 (Springer, Berlin, Germany; New York, USA, 1971).

[4] D. N. Spergel et al., Astrophys. J. Suppl. 170, 377 (2007), [arXiv:astro-ph/0603449]

[5] E. Komatsu et al., 2008, arXiv:0803.0547 [astro-ph]

[6] Planck, http://www.rssd.esa.int/.

[7] J. M. Bardeen, Phys. Rev. D 22, 1882 (1980).

[8] R. Durrer and N. Straumann, Helv. Phys. Acta 61, 1027 (1988).

[9] R. Durrer, Fund. Cosmic. Phys. 15, 209 (1994), [arXiv:astro-ph/9311041]

[10] V. Mukhanov, H. Feldman, and R. Brandenberger, Phys. Rep. 215, 203 (1992).

[11] G. I. Rigopoulos and E. P. S. Shellard, Phys. Rev. D 68, 123518 (2003), [arXiv:astro-ph/0306620]

[12] K. A. Malik and D. Wands, Class. Quant. Grav. 21, L65 (2004), [arXiv:astro-ph/0307055]

[13] F. Vernizzi, Phys. Rev. D 71, 061301 (2005), [arXiv:astro-ph/0411463]

[14] W. Hu and N. Sugiyama, Astrophys. J. 444, 489 (1995), [arXiv:astro-ph/9407093]

[15] W. Hu and M. White, Phys. Rev. D 56, 596 (1997), [arXiv:astro-ph/9702170].

[16] C.-P. Ma and E. Bertschinger, Astrophys. J. 455, 7 (1995), [arXiv:astro-ph/9506072].

[17] U. Seljak and M. Zaldarriaga, Cmbfast.

[18] A. Lewis and A. Challinor, Camb.

[19] J. Maldacena, JHEP 0305, 013 (2003), [arXiv: astro-ph/0210603]

[20] F. Bernardeau, T. Brunier, and J.-P. Uzan, Phys. Rev. D 69, 063520 (2004), [arXiv:astro-ph/0311422].

[21] S. Weinberg, Phys. Rev. D 72, 043514 (2005), [arXiv:hep-th/0506236]

[22] M. S. Sloth, Nucl. Phys. B 748, 149 (2006), [arXiv:astro-ph/0604488]

[23] M. S. Sloth, Nucl. Phys. B 775, 78 (2007), [arXiv:hep-th/0612138] 
[24] F. Bernardeau and J.-P. Uzan, Phys. Rev. D 66, 103506 (2002), [arXiv:hep-ph/0207295]

[25] F. Bernardeau and J.-P. Uzan, Phys. Rev. D 67, 121301 (2003), [arXiv:astro-ph/0209330]

[26] N. Bartolo, E. Komatsu, S. Matarrese, and A. Riotto, Phys. Rep. 402, 103 (2004), [arXiv:astro-ph/0406398]

[27] D. Seery and J. E. Lidsey, JCAP 0509, 011 (2005), [arXiv:astro-ph/0506056].

[28] F. Vernizzi and D. Wands, JCAP 0605, 019 (2006), [arXiv:astro-ph/0603799]

[29] G. I. Rigopoulos, E. P. S. Shellard, and B. J. W. van Tent, Phys. Rev. D 73, 083522 (2006), [arXiv:astro-ph/0506704].

[30] F. Arroja, S. Mizuno, and K. Koyama, 2008, [arXiv:0806.0619]

[31] D. Langlois and S. Renaux-Petel, JCAP 0804, 017 (2008), [arXiv:0801.1085]

[32] D. Langlois, S. Renaux-Petel, D. A. Steer, and T. Tanaka, 2008, [arXiv:0806.0336]

[33] E. Komatsu, D. N. Spergel, and B. D. Wandelt, Astrophys. J. 634, 14 (2005), [arXiv:astro-ph/0305189]

[34] P. Creminelli, L. Senatore, and M. Zaldarriaga, JCAP 0703, 019 (2007), [arXiv: astro-ph/0606001]

[35] A. P. S. Yadav, E. Komatsu, and B. D. Wandelt, Astrophys. J. 664, 680 (2007), [arXiv: astro-ph/0701921]

[36] A. P. S. Yadav and B. D. Wandelt, (2007), [arXiv:0712.1148]

[37] A. R. Cooray and W. Hu, Astrophys. J. 534, 533 (2000), [arXiv:astro-ph/9910397].

[38] D. M. Goldberg and D. N. Spergel, Phys. Rev. D 59, 103002 (1999), [arXiv:astro-ph/9811251]

[39] P. G. Castro, Phys. Rev. D 67, 044039 (2004), [arXiv:astro-ph/0212500]

[40] P. Serra and A. Cooray, (2008), [arXiv:0801.3276].

[41] N. Aghanim, S. Majumdar, and J. Silk, 2007, [arXiv:0711.0518]

[42] M. Bruni, S. Matarrese, S. Mollerach, and S. Sonego, Class. Quant. Grav. 14, 2585 (1997), [arXiv:gr-qc/9609040]

[43] K. Nakamura, Prog. Theor. Phys. 117, 17 (2007), [arXiv:gr-qc/0605108]

[44] C. Pitrou, J.-P. Uzan, and F. Bernardeau, Phys. Rev. D 78, 063526 (2008), [arXiv:0807.0341]

[45] C. Pitrou, Class. Quant. Grav. 24, 6127 (2007), [arXiv:0706.4383]

[46] W. Hu, D. Scott, and J. Silk, Phys. Rev. D 49, 648 (1994), [arXiv:astro-ph/9305038]

[47] K. Tomita, Phys. Rev. D 71, 083504 (2005), [arXiv:astro-ph/0501663]

[48] N. Bartolo, S. Matarrese, and A. Riotto, JCAP 0606, 024 (2006), [arXiv: astro-ph/0604416]

[49] N. Bartolo, S. Matarrese, and A. Riotto, JCAP 0701, 019 (2007), [arXiv:astro-ph/0610110]

[50] C. Pitrou and J.-P. Uzan, Phys. Rev. D 75, 087302 (2007), [arXiv:gr-qc/0701121]

[51] D. Langlois and F. Vernizzi, Phys. Rev. Lett. 95, 091303 (2005), [arXiv:astro-ph/0503416]

[52] D. Langlois and F. Vernizzi, Phys. Rev. D 72, 103501 (2005), [arXiv:astro-ph/0509078]

[53] A. Challinor and A. Lasenby, Astrophys. J. 513, 1 (1999), [arXiv:astro-ph/9804301]

[54] A. Challinor, Gen. Rel. Grav. 32, 1059 (2000), [arXiv:astro-ph/9903283]

[55] T. Gebbie and G. F. R. Ellis, Annals Phys. 282, 285 (2000), [arXiv:astro-ph/9804316]

[56] T. Gebbie, P. Dunsby, and G. Ellis, Annals Phys. 282, 321 (2000), [arXiv:astro-ph/9904408]

[57] R. Maartens, T. Gebbie, and G. Ellis, Phys. Rev. D 59, 083506 (1999), [arXiv:astro-ph/9808163].

[58] C. G. Tsagas, A. Challinor, and R. Maartens, (2007), [arXiv:0705.4397]

[59] G. F. R. Ellis and H. van Elst, (1998), [arXiv:gr-qc/9812046]

[60] M. Bruni, G. F. R. Ellis, and P. K. S. Dunsby, Class. Quant. Grav. 9, 921 (1992).

[61] B. Osano, C. Pitrou, P. Dunsby, J.-P. Uzan, and C. Clarkson, JCAP 0407, 003 (2007), [arXiv:gr-qc/0612108]

[62] K. Enqvist, J. Hogdahl, S. Nurmi, and F. Vernizzi, Phys. Rev. D 75, 023515 (2007), [arXiv: gr-qc/0611020]

[63] S. Bildhauer, Class. Quant. Grav. 6, 1171 (1989).

[64] A. Kosowsky, Annals Phys. 246, 49 (1996), [arXiv:astro-ph/9501045]

[65] A. Stebbins, 2007, [arXiv:astro-ph/0703541]

[66] C. W. Misner, K. S. Thorne, and J. A. Wheeler, Gravitation (Freeman, San Francisco, USA, 1973).

[67] J. Lesgourgues and S. Pastor, Phys. Rept. 429, 307 (2006), [arXiv:astro-ph/0603494]

[68] G. Dautcourt and K. Rose, Astronomische Nachrichten 13 (1978).

[69] K. S. Thorne, Rev. Mod. Phys. 52, 299 (1980).

[70] J.-P. Uzan, Class. Quant. Grav. 15, 1063 (1998), [arXiv:gr-qc/9801108]

[71] J. Martin-Garcìa, "xact and xpert", 2004.

[72] R. Wald, General Relativity (University of Chicago Press, Chicago, USA, 1984).

[73] P. Peter and J.-P. Uzan, Cosmologie primordiale (Belin, Paris, France, 2005).

[74] H. Kodama and M. Sasaki, Prog. Theor. Phys. Suppl. 78, 1 (1984). 
[75] K. A. Malik and D. R. Matravers, (2008), [arXiv:0804.3276]

[76] M. Hillery, R. F. O'Connell, M. O. Scully, and E. P. Wigner, Phys. Rep. 106, 121 (1984).

[77] N. Deruelle, J. Katz, and J.-P. Uzan, Class. Quant. Grav. 14, 421 (1997), [arXiv:gr-qc/9608046]

[78] E. Gourgoulhon, (2007), [arXiv:gr-qc/0703035]

[79] J. Portsmouth and E. Bertschinger, 2004, [arXiv:astro-ph/0412094]

[80] D. I. Nagirner and J. Poutanen, (2001), [arXiv:astro-ph/0108357]

[81] F. K. Hansen and P. B. Lilje, Mon. Not. Roy. Astron. Soc. 306, 153 (1999), [arXiv:astro-ph/9901066]

[82] A. D. Challinor and A. N. Lasenby, 1997, [arXiv:astro-ph/9711161]

[83] N. Itoh, Y. Kohyama, and S. Nozawa, Astrophys. J. 502, 7 (1998), [arXiv:astro-ph/9712289]

[84] S. Nozawa, N. Itoh, Y. Suda, and Y. Ohhata, 2005, [arXiv:astro-ph/0507466]

[85] A. Kompaneets, JETP 4, 730 (1957).

[86] S. Dodelson and J. M. Jubas, Astrophys. J. 439, 503 (1995), [arXiv:astro-ph/9308019]

[87] M. Beneke and C. Fidler, Phys.Rev. D82, 063509 (2010), [arXiv:1003.1834]

[88] D. Psaltis and F. K. Lamb, 1997, [arXiv:astro-ph/9706017].

[89] A. Challinor, Phys. Rev. D 62, 043004 (2000), [arXiv:astro-ph/9911481]

[90] W. Hu, Astrophys. J. 529, 12 (2000), [arXiv:astro-ph/9907103]

[91] U. Seljak and M. Zaldarriaga, Phys. Rev. Lett. 78, 2054 (1997), [arXiv:astro-ph/9609169].

[92] M. Zaldarriaga and U. Seljak, Phys. Rev. D 55, 1830 (1997), [arXiv:astro-ph/9609170].

[93] W. Hu, U. Seljak, M. J. White, and M. Zaldarriaga, Phys. Rev. D 57, 3290 (1998), [arXiv:astro-ph/9709066]

[94] V. Acquaviva, N. Bartolo, S. Matarrese, and A. Riotto, Nucl. Phys. B 667, 119 (2003), [arXiv:astro-ph/0209156].

[95] K. Nakamura, 2008, [arXiv:0804.3840]

[96] C. Pitrou, Dynamique non-linéaire et anisotropie primordiale en cosmologie, $\mathrm{PhD}$ thesis, Université Paris VI, Pierre et Marie Curie, 2008.

[97] D. Brizuela, J. M. Martin-Garcia, and G. A. Mena Marugan, (2008), [arXiv:0807.0824]. 